\title{
Near-wall streak modification by spanwise oscillatory wall motion and drag-reduction mechanisms
}

\author{
Emile Touber $\dagger$ and Michael A. Leschziner \\ Department of Aeronautics, Imperial College London, South Kensington Campus, London SW7 2AZ, UK
}

(Received 8 June 2011; revised 3 October 2011; accepted 14 November 2011;

first published online 16 January 2012)

Direct numerical simulations for fully developed channel flow, subjected to oscillatory spanwise wall motion, have been performed and analysed in an effort to illuminate the fundamental mechanisms responsible for the reduction in turbulent friction drag, observed to result from the spanwise wall motion. A range of statistical data are discussed, including second-moment budgets, joint-probability-density functions, enstrophy and energy-spectra maps. Structural features are also investigated by reference to the response of streak properties to the oscillatory forcing. The unsteady cross-flow straining is shown to cause major spanwise distortions in the streak nearwall structures, leading to a pronounced reduction in the wall-normal momentum exchange in the viscous sublayer, hence disrupting the turbulence contribution to the wall shear stress. The response of the streaks, in terms of their periodic reorientation in wall-parallel planes, the decline and recovery of their intensity during the cyclic actuation, and their wall-normal coherence, is shown to be closely correlated with the temporal variation of the shear-strain vector. Furthermore, a modulating 'top-to-bottom' effect, associated with large-scale outer-layer structures, is highlighted and deemed responsible for the observed reduction in the actuation efficiency as the Reynolds number is increased.

Key words: drag reduction, turbulence control, turbulent boundary layers

\section{Introduction}

Interest in methods that suppress near-wall turbulence in boundary layers is driven by pressure to reduce friction drag on immersed streamlined bodies and head losses in ducts and pipes. There is a broad consensus that this suppression requires a disruption and weakening of the quasi-organized wall-normal motions that are associated with quasi-streamwise vortices and thus are ultimately responsible for the formation of alternately high-speed and low-speed streaks in the viscosity-affected near-wall layer. It is this correlation between the wall-normal and streamwise-velocity fluctuations that gives rise to turbulent shear stress.

It is well established, based on two-point-correlation data, that the length of the streaks in fully established channel flow or a canonical boundary layer is of the order of $10^{3}$ wall units (Kreplin \& Eckelmann 1979), and that the streaks are separated by a

$†$ Email address for correspondence: e.touber@imperial.ac.uk 
distance of the order of $10^{2}$ wall units (Schraub \& Kline 1965; Kline et al. 1967; Kim, Kline \& Reynolds 1971). There is no complete agreement, however, on the precise mechanisms responsible for the formation and sustenance of these quasi-organized structures. Conventional paradigms, mostly derived from low-Reynolds-number direct numerical simulation (DNS) results, focus on the quasi-streamwise vortices as being the primary dynamical structures responsible for streak formation and turbulence generation (Kravchenko, Choi \& Moin 1993; Jimenez \& Pinelli 1999), and these are interpreted by some as being the tails of hairpin vortices (Zhou et al. 1999; Adrian, Meinhart \& Tomkins 2000). Several conceptual models describe a 'regeneration' or 'autonomous' cycle (Hamilton, Kim \& Waleffe 1995; Panton 1997; Waleffe \& Kim 1997; Jimenez \& Pinelli 1999; Schoppa \& Hussain 2002; Kim 2011), wherein new vortical structures are generated by low-speed-streak instability, nonlinear amplification and breakdown, with the new vortical structures regenerating new streaks. In contrast, recent linear analyses (Chernyshenko \& Baig 2005; Lockerby, Carpenter \& Davies 2005) do not rely directly on the action of vortices, and suggest that the streaks arise from the amplification of wall-normal velocity or vorticity perturbations, with the optimal (maximum-amplification) state characterized by streaks that are separated by distances comparable to those derived from DNS, i.e. of the order of $10^{2}$ wall units, although the analysis suggests a significant variation in the inter-streak distance with the distance from the wall. At elevated Reynolds numbers, the above near-wall interactions are further complicated by the increasingly important role played by a separate set of 'super-streaks' in the outer (log-law) region of the boundary layer, which manifest themselves as skin-friction footprints, and which are also observed, experimentally, to interact with, or 'modulate', the near-wall streaks in the viscosityaffected layer (Marusic, Mathis \& Hutchins 2010; Hutchins et al. 2011). An important implication of these 'top-down' mechanisms is that a wall-based control strategy directed at suppressing near-wall turbulence may be less effective as the Reynolds number increases. Indeed, this is suggested by the DNS of Ricco \& Quadrio (2008) at $R e_{\tau}=400$, the present study at $R e_{\tau}=500$ and recent large-eddy simulation (LES) results reported by Touber \& Leschziner (2011) at $R e_{\tau}=1000$.

Except for very low values of the Reynolds number (typically $R e_{\tau}=150$ in a channel), at which the near-wall turbulence is barely sustained, targeting individual streaks, vortices or wall-normal fluctuations, say, with closed-loop control inducing local surface deformations or blowing and suction (Choi, Moin \& Kim 1994; Lee et al. 1997; Rathnasingham \& Breuer 1997; Kang \& Choi 2000; Yoshino, Suzuki \& Kasagi 2003), is not a viable proposition. Rather, any practically relevant control strategy has to be global in nature, encompassing extensive portions of or the whole of the boundary layer. A demonstrably effective approach is to impose oscillatory in-plane wall motions, this being either spatially uniform or in the form of waves. In the case of a conducting fluid, a broadly equivalent alternative is to generate an oscillatory near-wall layer by Lorentz forcing (e.g. Du, Symeonidis \& Karniadakis 2002; Pang \& Choi 2004).

Early observations on the consequences of imposing a pressure-gradient-induced spanwise near-wall motion on a previously streamwise-aligned boundary layer arise from experimental studies by Bradshaw \& Pontikos (1985), demonstrating a drop in shear stress and skin friction due to rising three-dimensional skewing. Related physical scenarios, involving either three-dimensional boundary layers or channel flow, were studied experimentally by Driver \& Hebbar (1987), Eaton (1995) and computationally by Sendstad \& Moin (1992), among others, the last two including discussions of the effect of strain-induced distortions on the quasi-streamwise vortices associated 
with sweeps and ejections close to the wall. A DNS study by Howard \& Sandham (2000) of a channel flow at $R e_{\tau}=180$, subjected to a suddenly imposed spanwise, unidirectional wall motion, shows the decrease in drag to be linked, statistically, to the damping of turbulence energy and its production near the wall, and demonstrated that this suppression is maintained for only a short period of time after the forcing starts, while the flow is subjected to strong unsteady skewing. This study also examines the effect of the spanwise motion on the structural properties of streamwise eddies and the streaks, highlighting in particular, the fact that the skewing motion results in a weakening and flattening of the quasi-streamwise vortices.

The realization that a steady-state reduction in drag is conditional on unsteady transverse straining within a thin near-wall layer led Jung, Mangiavacchi \& Akhavan (1992) to explore, by means of DNS, the benefits of oscillatory spanwise wall motion, thus preventing it from progressively thickening beyond a particular height, dictated by the parameters of the actuation (the frequency of oscillation, in particular). This study, for a channel flow at $R e_{\tau}=200$, was the first to reveal the sensitive dependence of the magnitude of drag decrease on the oscillation frequency, and to demonstrate that an oscillation period $T^{+} \equiv T u_{\tau}^{2} / \nu$ of the order of $10^{2}$ leads to a strong decline in the level of the Reynolds stresses and the strength of the near-wall streak, the convergence to the reduced-drag state occurring within four oscillation periods. In a related study, Akhavan, Jung \& Mangiavacchi (1993) first discuss the interaction of the spanwise forcing with the near-wall quasi-streamwise vortices and low-speed streaks, highlighting the disruption of the regeneration process by weakening the wall-normal fluctuations, arguments supported by the later experimental study of Laadhari, Skandaji \& Morel (1994) for a canonical boundary layer. The latter investigation also shows that the spanwise motion, chosen at the near-optimum forcing frequency $T^{+}=100$, results in a substantial reduction in the fluctuations contributing to the shear stress, in qualitative agreement with the earlier channel-flow DNS. Finally, based on weighted probability density functions (PDFs), Laadhari et al. (1994) conclude that, while both low-speed and high-speed motions are damped, the reduction is asymmetric, with the latter being less reduced, due to the increased probability of large sweeping events, presumably reflecting the relatively greater importance of top-down effects.

Much subsequent work, both experimental and computational, has been undertaken, especially over the past decade, to investigate a variety of actuation scenarios involving different types of in-plane wall motion, some combining transverse oscillations with streamwise travelling waves. Major studies in this area are those of Baron \& Quadrio (1995), Choi, DeBisschop \& Clayton (1998), Choi \& Graham (1998), Dhanak \& Si (1999), Quadrio \& Sibilla (2000), Choi \& Clayton (2001), Choi (2002), Choi, $\mathrm{Xu} \&$ Sung (2002), Di Cicca et al. (2002), Karniadakis \& Choi (2003), Quadrio \& Ricco (2003), Quadrio \& Ricco (2004), Quadrio \& Ricco (2011), Ricco (2004) and Quadrio, Ricco \& Viotti (2009), the paper by Karniadakis \& Choi (2003) being a major review article. The primary objective of several of the more recent efforts, especially by Quadrio and co-workers, was to identify optimal actuation scenarios, principally in terms of overall drag-reduction levels, while others aimed preferentially to shed light on the underlying near-wall dynamics and kinematics responsible for the observed drag reduction. A common aspect of almost all computational studies is the low Reynolds number of the flows examined. With very few exceptions, such as the works of Choi \& Graham (1998), Quadrio \& Sibilla (2000) and Choi et al. (2002) on pipe flow subjected to azimuthal wall oscillations and the simulations of Ricco \& Quadrio (2008) for channel flow at $R e_{\tau}=400$, computational investigations focused on channel flow at $R e_{\tau}=200$ or less, while experimental efforts examined the response 
of canonical boundary layers to streamwise-uniform spanwise oscillations, typically at $R e_{\tau}$ of the order of 600 , corresponding to a momentum-thickness Reynolds number of the order of 1400. This sharp division in preferences for one geometry relative to the other is rooted in the much greater ease with which a channel flow can be set up computationally, while a boundary layer is a much simpler option in an experimental setting. Although this difference might appear to be of subordinate importance, in an environment in which the near-wall region is the subject of study, the influence of outer structures on the near-wall behaviour can be expected to differ. To the best of the authors' knowledge, the influence of these structures has not been taken into consideration or analysed explicitly in the context of drag reduction by spanwise wall motion.

The investigations of Choi et al. (1998), Di Cicca et al. (2002), Choi (2002), Choi \& Clayton (2001) and Ricco (2004) were all experimental in nature, the first utilizing particle-image velocimetry, the second a hydrogen-bubble technique and the last three hot-wire anemometry, and all targeting boundary layers at similar values of the Reynolds number. Ricco (2004) reports drag-reduction levels of up to $39 \%$, at a near-optimum oscillation period in combination with high values of the wall-velocity amplitude, provides measurements of maximum streak orientation at various forcing conditions, and presents data that suggest a weakly increasing inter-streak distance and decreasing streak length with decreasing drag. Di Cicca et al. (2002) show that oscillatory forcing provokes a thickening of the viscous sublayer, an upward shift of the log-law, and a significant reduction in the streamwise and wall-normal turbulence intensities, associated with a reduction in turbulence-energy production. Based on conditionally sampled statistics, Di Cicca et al. (2002) also argue, in agreement with Laadhari et al. (1994), that the major contributor to the turbulence attenuation is the preferential weakening of the low-speed streaks, thus leading to a disruption of the regeneration process. The experimental work of Choi \& Clayton (2001) and Choi (2002) attempts to explain the drag-reduction mechanism by considering the interaction of the vortex sheet associated with the Stokes layer with the quasi-streamwise vortices. Specifically, they develop a conceptual model that rests on the net generation of negative spanwise vorticity that leads to a reduction in the mean-velocity gradient at the wall, and hence in the measured $45 \%$ reduction in wall friction. However, the model is open to doubt, because it appears to be based on the assumption that the visualized orientation of the streaks is closely linked to the direction of wall motion, while in reality, it is the direction of the shear-strain vector that dictates the orientation of the streaks. As a more general point, the question might be raised whether a focus on the fairly weak vortical structures in the near-wall region, rather than on the streaks and the wall-normal motions provoking the streaks, is the most promising route to identifying the pertinent fundamental interactions between the oscillatory forcing and the near-wall structures. The measurements of Choi (2002) of the near-wall fluctuations and the drag reduction process led him further to conclude that the drag-reduction process is driven primarily by a strong diminution in the intensity and duration of sweep events. While this chimes with the concept of Ricco (2004) of forcing-induced 'shielding' of the wall, Choi's own PDF profiles of the streamwise fluctuations very close to the wall indicate, in common with observations by Laadhari et al. (1994), an increasing predominance, or evidence, of high-intensity sweeps and large high-speed streamwise fluctuations in the presence of forcing, contradicting the concept of 'shielding' and strengthening the inference that the outer structures gain in relative influence as the near-wall streaks weaken. 
A rare computational investigation that aims specifically at shedding light on the interactions between streaks and quasi-streamwise vortices of the type examined in some of the above experimental studies is that of Choi et al. (2002). One of several cases considered is a pipe flow at $R e_{\tau}=150$ subjected to azimuthal oscillations at $T^{+}=116$ and 174. This study presents plots of phase-averaged data, sampled conditionally on the quasi-streamwise vortices, together with streamwisevelocity fluctuations that identify surrounding streaks. As remarked already, the focus on vortices, rather than streaks, is perhaps open to question, because the vortices themselves are rather weak and ill-defined, in contrast with the streaks and associated ejections and sweeps, within which the vorticity is concentrated and high. Nevertheless, the results reveal that high-speed streaks tend to be swept below their low-speed counterparts by the wall motion within the first half of the oscillation, causing the low-speed streaks to be initially lifted, and then to weaken substantially in the second half of the oscillation. While this does not really explain the root of the turbulence attenuation process, it does appear to point, yet again, to the link between the oscillation-induced disruption of the near-wall regeneration process to the attenuation of the low-speed streaks. A somewhat similar route was taken much later by Yakeno, Hasegawa \& Kasagi (2010), in respect analysing conditionally averaged vortical structures in the spanwise plane, based on DNS for a channel flow, as an aid to interpreting a quadrant analysis of shear-stress contributions.

In a series of articles, extending over some 15 years, Quadrio and co-workers have explored various aspects of wall-motion-induced drag reduction by means of DNS. Major characteristics of these studies include the focus on low-Reynolds-number channel flow, at $R e_{\tau}=200$, the orientation towards maps of gross and net dragreduction levels as the principal outcome, and the aim to identify, in a compact manner based on appropriate scaling, optimum forcing conditions that yield maximum drag-reduction levels for different forcing conditions. In the earliest work of this group, Baron \& Quadrio (1995) report DNS results for $R e_{\tau}=200$ and $T^{+}=100$. One interesting aspect of the study, if only as a matter of historical interest, is that the simulation was performed with a finite-difference mesh of less than $4 \times 10^{5}$ grid points covering a minimal-channel domain: a resolution that is significantly coarser than that used in the much earlier study of Jung et al. (1992), and certainly regarded as coarse by today's standards. Yet, the simulation yielded an entirely plausible representation, certainly in broad terms, of the effects of the forcing, and this extends to the turbulence-intensity components, and even to the budget of the turbulence energy reported by the authors. In particular, the reduction in drag obtained was $40 \%$, which is close to the level predicted with the far finer grids that would be expected in current simulations. Subsequent studies, increasingly broad in the range of the actuation parameters investigated, but still focusing on pure spanwise oscillations in channel flow, are those of Quadrio \& Sibilla (2000), Quadrio \& Ricco (2003, 2004) and Ricco \& Quadrio (2008). The focus of the study by Quadrio \& Ricco (2003) was the transient response of a channel flow at $R e_{\tau}=200$ to the sudden imposition of the spanwise wall motion, with attention devoted primarily to the skin-friction behaviour. It is noted here, as an aside, that in any study of the transient adjustment (including that of Howard \& Sandham 2000) sampling is restricted to the number of nodes in any statistically homogeneous plane; hence, the convergence of statistics (especially budgets) may be incomplete, and this is especially problematic at elevated Reynolds numbers. Subject to this uncertainty, Quadrio \& Ricco (2003) show that the wall shear stress reaches its equilibrium level within three or four oscillations, in agreement with observations by Jung et al. (1992), and provide data for the Reynolds 
stresses and production rates of the streamwise and spanwise stresses that indicate a non-monotonic behaviour of the turbulence reduction during the transient approach to the equilibrium level. A similar study by Xu \& Huang (2005) also examines transient features of a channel flow, at $R e_{\tau}=173$, but over only the first two oscillation periods. Exceptionally, results are included for the pressure-strain contributions of the budgets for some of the second moment, and their diminution throughout the transient adjustment is argued to play an important role in the drag-reduction process. In agreement with the observations by Quadrio \& Ricco (2003), Xu \& Huang (2005) demonstrate high production rates of spanwise fluctuations during the transient process, leading to an intensification of the spanwise intensity during the initial phase following the onset of the spanwise motion. Importantly, however, Quadrio \& Ricco (2003) observe that the long-term average of the production rate vanishes; this is shown in the present study not to be the case.

The investigation of Quadrio \& Ricco (2004) focuses on the quantification of the maximum gross as well as net drag reduction (or rather associated power levels) over a wide range of wall velocity and actuation periods, for channel flow at $R e_{\tau}=200$, with the results conveyed in drag-reduction maps. High values of gross drag reduction, of the order of $35-45 \%$, are achieved at $T^{+}$around 100-150 in conjunction with high wall velocities, broadly compatible with the experimental measurements of Ricco (2004), the latter albeit in a canonical boundary layer at higher Reynolds number. A principal result in the study of Quadrio \& Ricco (2004) is the fact that the dragreduction levels, arising for actuation periods $T^{+}$below 150, collapse on a line in a plot that relates the drag-reduction level to a parameter $S^{+}$, first proposed by Choi et al. (2002) and combining $T^{+}$, the wall-scaled amplitude of the wall velocity, the penetration depth of the Stokes layer and the maximum spanwise acceleration at a particular distance from the wall. For conditions in which $T^{+}$significantly exceeds the optimum value, drag reduction is far lower and possibly even negative. Subsequent work by Ricco \& Quadrio (2008) recasts the data available in the literature in the form of the drag reduction $D R$ vs $S^{+}$, and this confirms, subject to a non-negligible amount of scatter, the applicability of the scaling for low-Reynolds-number values when $T^{+}<150$. However, Ricco \& Quadrio (2008) also show that the drag-reduction levels at various oscillation periods drop by around 5\% (from a maximum of $32 \%$ to $27 \%$ ) as $R e_{\tau}$ increases from 200 to 400 , leading to a non-unique $D R=f\left(S^{+}\right)$ correlation and reinforcing the supposition that top-down effects become increasingly influential as the Reynolds number increases. In their most recent major studies, Quadrio et al. (2009) and Quadrio \& Ricco (2011) turn their attention to forcing with streamwise waves of spanwise wall motion. Here again, the primary target is to derive drag-reduction maps, in this case for a range of spanwise-oscillation frequencies and streamwise wavenumbers at $R e_{\tau}=200$. The map so derived shows intriguing features, including the fact that regions of high drag-reduction levels lie on either side of a narrow inclined band identifying drag increase. Moreover, optimum combinations of forcing frequency and streamwise wave length are shown to result in maximum gross drag-reduction levels $\sim 10 \%$ higher than those that can be achieved purely by uniform spanwise wall oscillations.

By way of a summary of the present state of the art, it is fair to reiterate, first, that much of the present knowledge on the influence of purely spanwise-oscillatory motion derives from flows at low values of the Reynolds number, especially in the case of computational studies. While there is some evidence (Ricco \& Quadrio 2008) that increasing the Reynolds number decreases the drag-reduction effectiveness of the forcing, little is reported beyond the overall drag-reductions levels, and the 
applicability of other conclusions to higher, practically pertinent values is uncertain. Connected to this question is the need to understand and quantify the top-down influence of outer super-streaks on the near-wall physics, an issue that has not been addressed so far. Second, it is the case that the majority of the studies, especially computational ones, focus on global aspects of the effects of spanwise wall oscillation: overall drag reduction, transient adjustment to the long-term state, the role of the Stokes layer thickness, the sensitivity to operational parameters and scaling laws. Third, while most studies report at least some statistical information, including Reynolds stresses and, in some instances, contributions to budgets, the information is fragmentary, especially in respect of budgets, and there is no clear view of how the various contributions to the budgets interact, across the entire set of Reynolds stresses, for the forced flow to end up in an low-drag equilibrium state. Fourth, while there have clearly been substantive efforts to illuminate the fundamental mechanisms at play, some by experimental means and others by creative processing of computational data, the details remain unclear. There is a measure of agreement on the preferential attenuation of the low-speed streaks and its connection to the disruption of the regeneration process, while intense sweep events, presumably originating from beyond the viscous sublayer, prevent the same level of attenuation of high-speed streamwise fluctuations near the wall. The details of structural mechanisms underlying the attenuation and disruption are unclear, however. Fifth, phase-averaged and conditionally averaged data that reveal the behaviour of streaks during the actuation cycle are very scarce. Images derived from phase-averaged fields, with sampling being conditional on the quasi-streamwise vortices rather than streaks, give support to the important role played by the weakening of the low-speed streaks through their deformation by the oscillatory motion. Finally, visualization experiments have been used as an aid to a conceptual model that is based on the interaction of the vorticity in the Stokes layer with the quasi-streamwise vortices, but the validity of the model is open to question.

It is against the above background that the present DNS-based study has been undertaken for a channel flow at $R e_{\tau}=500,2.5$ times the usual value for which detailed analyses have been presented. Apart from this point of difference, the study distinguishes itself from previous work by:

(a) the provision of complete budgets of the Reynolds stresses formed with the stochastic components, following their derivation from a triple decomposition of the velocity;

(b) the identification of important interactions within individual budgets and among budgets for different stresses;

(c) the inclusion of long-term-averaged and phase-averaged joint PDFs to illustrate the suppression of turbulence in different quadrants;

(d) the inclusion of an analysis that clarifies the level of phase dependence of the flow field and the skin friction;

(e) the presentation of enstrophy components and associated enstrophy production, so as to illustrate how the spanwise motion influences the components;

( $f$ ) the identification of the origin of the enhancement of the spanwise stochastic fluctuations in the presence of spanwise straining by reference to specific production fragments in the budget equations;

$(g)$ the clarification of the relationship between the streaks and the shear-strain angle; specifically, the dependence of the streak angle, formation and disruption on the rate of change of the shear-strain angle; 
(h) the presentation of streak-conditional phase-averaged fields that clarify the response of the streaks to the distortions provoked by the Stokes layer;

(i) the linkage of the statistical analysis to structural properties; and

(j) the identification of the influence of outer structures on the near-wall streaks and skin friction, including aspects of the former modulating the latter and thus reducing the drag-reduction effectiveness.

\section{Flow conditions and computational realization}

The configuration being considered is a fully developed (streamwise-periodic) channel flow. The principal value of the friction Reynolds number $R e_{\tau} \equiv u_{\tau} h / v$ is 500 , but some results are also presented for the values 200 and 1000 , where $h$ is the channel half height, $v$ the kinematic viscosity and $u_{\tau}^{2} \equiv v \mathrm{~d} \bar{u} /\left.\mathrm{d} y\right|_{y=0}$, with $\bar{u}$ the mean streamwise velocity. The flow is subjected to spanwise forcing at the periods $T^{+}=T u_{\tau}^{2} / \nu=100$ and 200 , where $u_{\tau}$ is the value pertaining to the unforced case. The former value complies with the generally accepted optimum oscillation period, while the latter has been included in order to bring out the response of some structural features that are too weakly delineated at $T^{+}=100$, because of the exceptionally strong suppression of near-wall turbulence.

The imposed spanwise forcing, on both walls, is described by

$$
W(t)=W_{m} \sin \left[\frac{2 \pi t}{T}\right] .
$$

Based on the unforced friction velocity, the wall-velocity amplitude is $W_{m}^{+}=$ $W_{m} / u_{\tau}=12$, where $u_{\tau}$ is, here again, the value for the unforced flow. The above values for $T^{+}$and $W_{m}^{+}$, when processed in conjunction with data derived from the Stokes layer, as explained in Choi et al. (2002) and Quadrio \& Ricco (2004), yield $S^{+}=0.29$ and 0.28 for $T^{+}=100$ and 200 , respectively. When forcing is introduced, the bulk Reynolds number is held at a constant level relative to the corresponding unforced flow. Hence, the friction Reynolds number changes (reduces) in the presence of forcing. Throughout this paper, $T^{+}, W_{m}^{+}$and $S^{+}$are normalized by the friction velocity of the unforced case. In all other cases, such a normalization is identified by the subscript or superscript 'nominal'. The absence of this qualifier indicates that normalization is based on the actual friction velocity.

The grid used to achieve the requisite resolution comprised $384 \times 256 \times 384$ cells, covering a channel section of $4 \pi h \times 2 h \times 2 \pi h$. The cell distances, scaled with the unforced friction velocity, were $\Delta x^{+}, \Delta y_{\min / \max }^{+}, \Delta z^{+}=17,0.7-6.2,8.3$. Using the stochastic dissipation rate of the turbulence energy, derived as part of the secondmoment budgets (see §7), allows the Kolmogorov length scale, and thus the ratio of cell size to this length scale, to be determined. With the cell size taken as $[\Delta x \times \Delta y \times \Delta z]^{1 / 3}$, the ratio varies between four at the wall to two close to the channel centre. This level of resolution is very similar to that adopted in other DNS studies for which grid-resolution details are reported. For example, Quadrio \& Ricco (2003) have used the cell dimensions $\Delta x^{+}, \Delta y_{\min / \max }^{+}, \Delta z^{+}=16,0.8-5.4,6.5$. The turbulence-energy budget obtained herein for the unforced flow at $R e_{\tau}=500$ was found to compare closely with that reported by Moser, Kim \& Mansour (1999).

A few further operational conditions, at $R e_{\tau}=200$ and 1000 , have also been examined as part of the present study. Results for $R e_{\tau}=200$ are only included in a few instances: first, to verify agreement with previous data underpinning the correlation $D R=f\left(S^{+}\right)$from Quadrio \& Ricco (2004), where $D R$ denotes the relative 
drag reduction and, second, to support the discussion of certain statistical properties for $R e_{\tau}=500$. Results for the highest value have been obtained from wall-resolved LES, using a grid resolution of $\Delta x^{+}, \Delta y_{\min }^{+}, \Delta z^{+}=40,1.3,20$ and employing a dynamic version of the Smagorinsky subgrid-scale model, and these are only included in respect of the overall drag-reduction levels, in order to support observations derived from lower-Reynolds-number values on the dependence of the drag reduction on the Reynolds number. The equivalent values of the bulk Reynolds number $\left(R e_{\text {bulk }}\right)$, based on mean velocity $\left(U_{b u l k}\right)$ and channel half-height $(h)$, are 3150, 9000 and 20000, respectively. The use of LES arguably poses a question mark against the accuracy of the data. However, a drag-reduction result now available from a fully resolving DNS for $T^{+}=100$, with $1056 \times 528 \times 1056$ (589 million) cells and mesh resolution $\Delta x^{+}, \Delta y_{\min / \max }^{+}, \Delta z^{+}=12,0.4-7,6$, is within $5 \%$ of the drag-reduction margin $(28 \%$ for the LES and $29 \%$ for the DNS at $\left.T^{+}=100\right)$ predicted by the corresponding LES. It may be argued, therefore, that the LES data give a credible first-order indication on the practically-important issue of the likely deterioration of the forcing effectiveness as the Reynolds number increases.

All simulations contributing to the present study have been performed with a variant of the general non-orthogonal-grid, block-structured, finite-volume method based on a fully co-located storage and realized in the in-house code STREAMLES for incompressible flows (Temmerman et al. 2003). The algorithm advances the velocity field in time by means of a fractional-step method incorporating fourthorder approximations for the fluxes and a third-order Gear-like scheme documented in Fishpool \& Leschziner (2009), the last shown to possess advantageous stability and accuracy properties, relative to a corresponding second-order time-advancement scheme. Zero divergence is secured by solving the pressure-Poisson equation, with second-order accuracy, combining the application of an implicit successive line overrelaxation (SLOR) method with a multigrid scheme. Pressure-velocity decoupling, arising from the fully collocated storage of the velocity and pressure, is counteracted by employing the, so-called, Rhie \& Chow (1983) interpolation scheme. Fishpool \& Leschziner (2009) demonstrate that the loss of energy due to artificial dissipation arising from the Rhie \& Chow interpolation is low. The code is fully parallelized using MPI, with predetermined grid blocks or subdomains assigned to individual processors. Typically, each simulation for $R e_{\tau}=500$ required $\sim 4000 \mathrm{CPU}$ hours, distributed across 64 processors, on an in-house SGI Altix ICE 8200 EX cluster.

\section{Global properties}

A global view of the effects of the forcing on the skin friction is conveyed by table 1 and figure 1. In the latter, the drag-reduction correlation proposed by Choi et al. (2002) and examined extensively by Quadrio \& Ricco (2004) for low-Reynoldsnumber flows forced at $T^{+}<150$ is identified by 'QR2004'. The results included for $R e_{\tau}=200$ illustrate that the present simulations yield, for close-to-optimum forcing, drag-reduction levels that are entirely compatible with previous data. As is seen from table 1 , increasing the forcing period quickly leads to a steep decline in effectiveness and this is reflected by the corresponding data points (open circles) lying well below the QR2004 line. At $R e_{\tau}=500$, there is a decline by $\sim 18 \%$ in the maximum dragreduction margin, relative to $R e_{\tau}=200$. This is broadly in line with a decline of around $15 \%$ reported by Ricco \& Quadrio (2008) when $R e_{\tau}$ is increased from 200 to 400. Results derived from the wall-resolved LES for $R e_{\tau}=1000$ (see §2) indicate a further deterioration, by $\sim 40 \%$. The shaded band around the best-fit line of the LES 


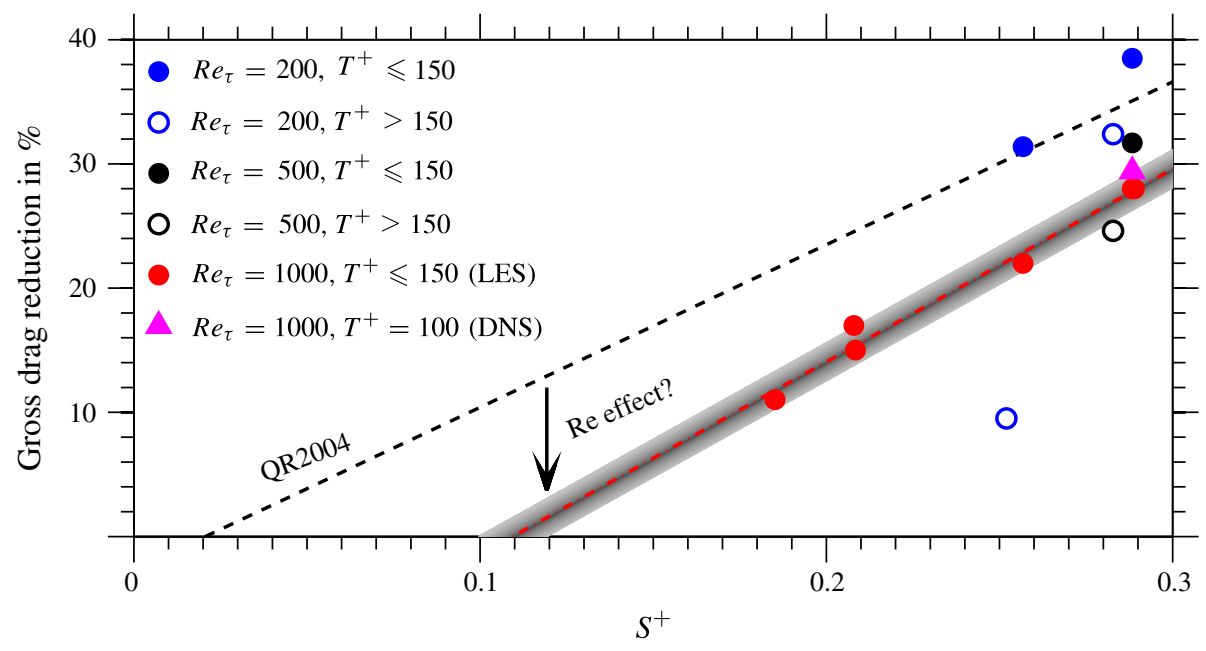

FIGURE 1. Correlation between the composite parameter $S^{+}$and the level of drag reduction. The line labelled 'QR2004' corresponds to the best-fit line $\left(\% D R=131 S^{+}-2.7\right)$ taken from Quadrio \& Ricco (2004). Data for $R e_{\tau}=1000$ are derived from LES, with one DNS exception. The shaded area corresponds to a $\pm 5 \%$ error in the maximum drag-reduction margin at $R e_{\tau}=1000, T^{+}=100$, suggested by the difference between the LES and DNS at these conditions.

$\begin{array}{lccccccc}\text { Nominal } R e_{\tau} & 200^{a} & & & & & 500^{b} \\ T^{+} & 50 & 100 & 200 & 400 & 1000 & 100 & 200 \\ \text { Drag reduction (\%) } & 31.4 & 38.5 & 32.4 & 9.5 & -6.3 & 31.7 & 24.6\end{array}$

TABLE 1. Gross drag-reduction levels from the DNS. a Actual values is 197 when unforced. ${ }^{\mathrm{b}}$ Actual values is 509 when unforced.

data points represents a $\pm 5 \%$ error margin, reflecting the difference between the LES and DNS results for $R e_{\tau}=1000$ and $T^{+}=100$, as explained in $\S 2$. The decline in the effectiveness of the forcing at the higher Reynolds number points to a diminishing influence of the processes in the viscous sublayer on the drag, and to an increasing impact of larger-scale sweeping events originating in the outer log-law region, as suggested by Laadhari et al. (1994). A fit to the present drag-reduction data, including that at $R e_{\tau}=1000$, allows the tentative proposal to be made that the drag-reduction margin achieved under similar $S^{+}$values declines roughly as $R e_{\tau}^{-0.2}$. This is broadly in line with a suggestion made by Choi et al. (2002), albeit one based on simulations at $R e_{\tau}$ of 400 and lower. Extrapolated to cruise-flight Reynolds numbers, $R e_{\tau}$ of the order of $10^{5}-10^{6}$ (with all of the risks of doing so acknowledged), this suggests a gross drag-reduction level of the order of $7-10 \%$.

The effect of the forcing on the streamwise-velocity profile, scaled with the actual as well as nominal (unforced) friction velocity, is conveyed by figure 2 at the close-tooptimum forcing period $T^{+}=100$. Again, purely by way of contrast to the conditions at lower Reynolds numbers on which most earlier studies focus, the corresponding profiles at $R e_{\tau}=200$ are also included. In both cases, but clearly less so for the higher Reynolds number, the forcing results in a significant thickening of the viscous sublayer 

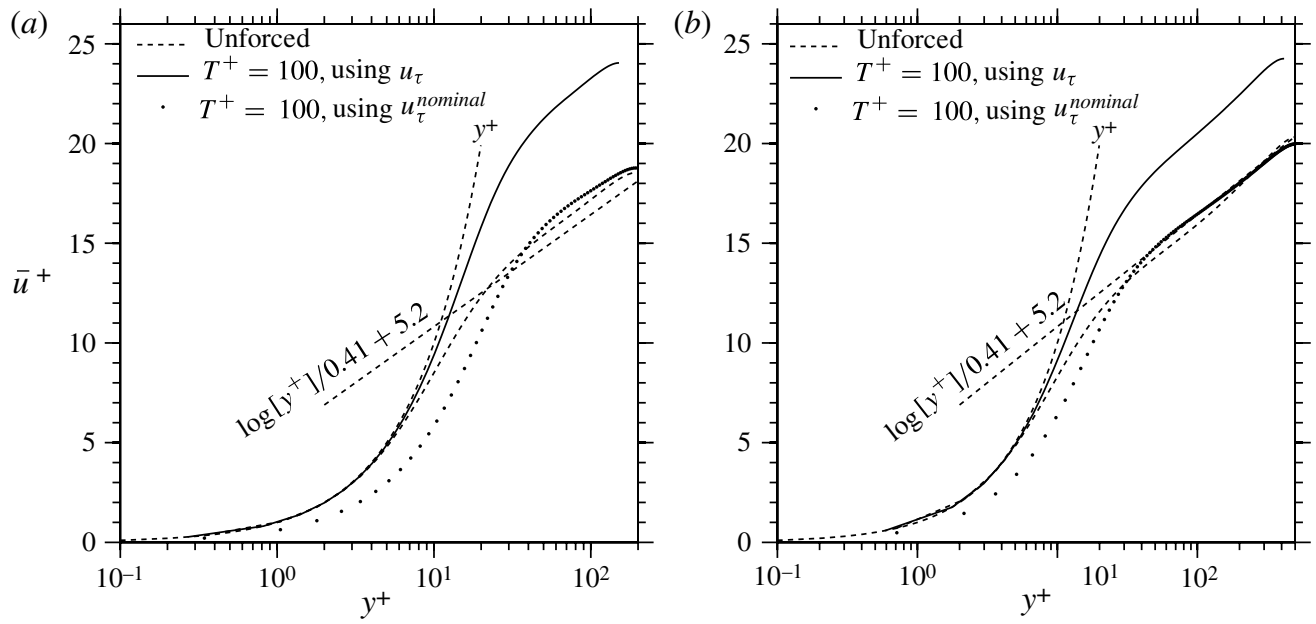

FIGURE 2. Mean streamwise-velocity profiles for the close-to-optimum forcing period for both $R e_{\tau}=200$ and 500. Profiles are scaled with both the nominal and actual friction velocity: (a) $R e_{\tau}=200 ;\left(\right.$ b) $R e_{\tau}=500$.

and a shortening and elevation of the log-law portion. Scaling by the nominal friction velocity brings out the fact that the major impact of the forcing is to significantly lower the velocity in the viscous layer, while the effect in the log-law portion is a very slight elevation. The overall effect of the forcing is thus to drive the flow towards the laminar state, a behaviour that is also observed to arise from a strong acceleration in a sink flow (Spalart 1986), in which the streaky near-wall structure is also observed to be damped.

\section{The stochastic field and its phase variations}

In any turbulent flow subjected to periodic excitation, the time-averaged fields agglomerate contributions that arise from periodic and stochastic motions. Thus, in order to investigate and understand the influence of the forcing on the turbulence field, it is necessary to separate carefully these two contributions. This is especially pertinent to the spanwise direction in which the Stokes layers creates very large oscillatory motions, and in which, in particular, the Stokes dissipation is much larger than the stochastic component.

The determination of the stochastic field requires the introduction of a triple decomposition of any flow property, say $a$ :

$$
a=\bar{a}+\underbrace{\tilde{a}+a^{\prime \prime}}_{a^{\prime}},
$$

where $\bar{a}$ is the time-averaged value of $a, \tilde{a}$ the difference between the ensembleaverage of $a$ at a given forcing phase (defined based on the forcing signal (2.1)) and the time-averaged value, and $a^{\prime \prime}$ the stochastic (turbulent) contribution. The total fluctuation is denoted $a^{\prime}$ and includes both the phase and stochastic fluctuations.

An important (and perhaps somewhat counterintuitive) aspect of the interaction between the forcing and the flow is the low-level phasewise variations of the velocity field and turbulence properties in the streamwise direction. Linked to this is the fact that the phase-averaged spanwise velocity differs little from the laminar solution. 

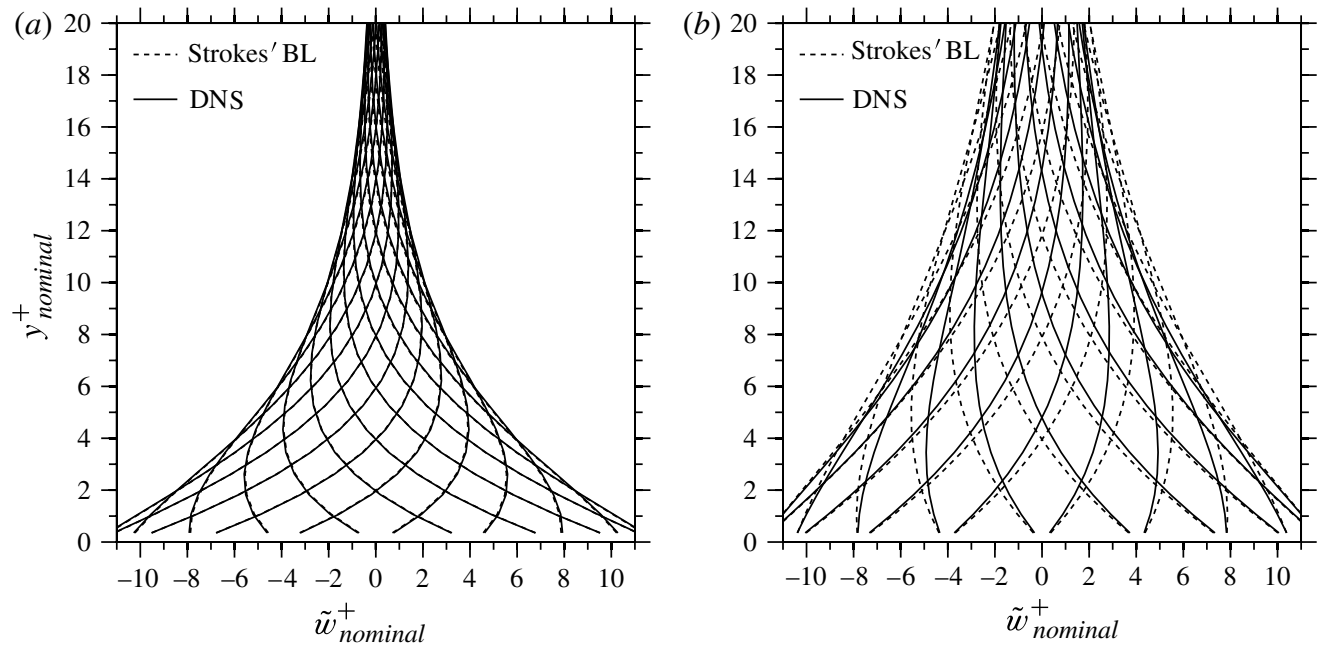

FIGURE 3. Stokes boundary layer and phase-averaged spanwise-velocity fluctuations at $\operatorname{Re}_{\tau}=200:(a) T^{+}=100 ;(b) T^{+}=400$.

Appendix A demonstrates that, in the present case of a channel flow, the phaseaveraged velocity fluctuations follow from

$$
\begin{gathered}
\frac{\partial \tilde{u}}{\partial t}=\frac{1}{R e} \frac{\partial^{2} \tilde{u}}{\partial y^{2}}+\frac{\partial}{\partial y}\left[\overline{u^{\prime \prime} v^{\prime \prime}}-\left\langle u^{\prime \prime} v^{\prime \prime}\right\rangle\right], \\
\tilde{v}=0, \\
\frac{\partial \tilde{w}}{\partial t}=\frac{1}{R e} \frac{\partial^{2} \tilde{w}}{\partial y^{2}}+\frac{\partial}{\partial y}\left[\overline{w^{\prime \prime} v^{\prime \prime}}-\left\langle w^{\prime \prime} v^{\prime \prime}\right\rangle\right],
\end{gathered}
$$

where the notation $\langle\cdot\rangle$ denotes phase-averaged quantities.

The right-most terms in the brackets in $(4.2 a)$ and (4.2c) invoke a modulation of the stress-tensor components $v^{\prime \prime}-u^{\prime \prime}$ and $v^{\prime \prime}-w^{\prime \prime}$ by the periodic forcing. It is shown in appendix A that, if this modulation is of the order of $\varepsilon$, the condition $\varepsilon \ll W_{m}^{+}\left(R e_{\tau}^{2} / R e_{b u l k}^{2}\right) / \sqrt{T^{+}}$must be satisfied for the Stokes motion to remain close to the laminar solution. This condition implies that, for sufficiently short forcing periods, the phase-averaged spanwise-velocity fluctuations can be considered equal to their laminar counterparts. This is illustrated in figure 3. For $R e_{\tau}=200$ and $W_{m}^{+}=12$, the DNS data give $\varepsilon \sim 10^{-5}$ and $\varepsilon \sim 10^{-4}$, for $T^{+}=100$ and $T^{+}=400$, respectively, while the constraint in (A 12) requires $\varepsilon$ to be significantly smaller than $10^{-3}$. It is not surprising, therefore, that a good match is obtained between the DNS and the laminar solution, as is demonstrated in figure 3 for $T^{+}=100$, while departures are only noticeable when $T^{+}$is substantially larger than the optimum value, in which case the Stokes layer extends well beyond the viscous sublayer.

An important consequence of the above is that the stochastic streamwise fluctuations are very close to the respective total fluctuations, while there is no such difference at all in the case of the wall-normal fluctuations. Moreover, any extra stress-production terms associated with phase-averaged strains are very small, with exception of the production of the spanwise normal stress, as will be demonstrated later by reference to second-moment budgets. 

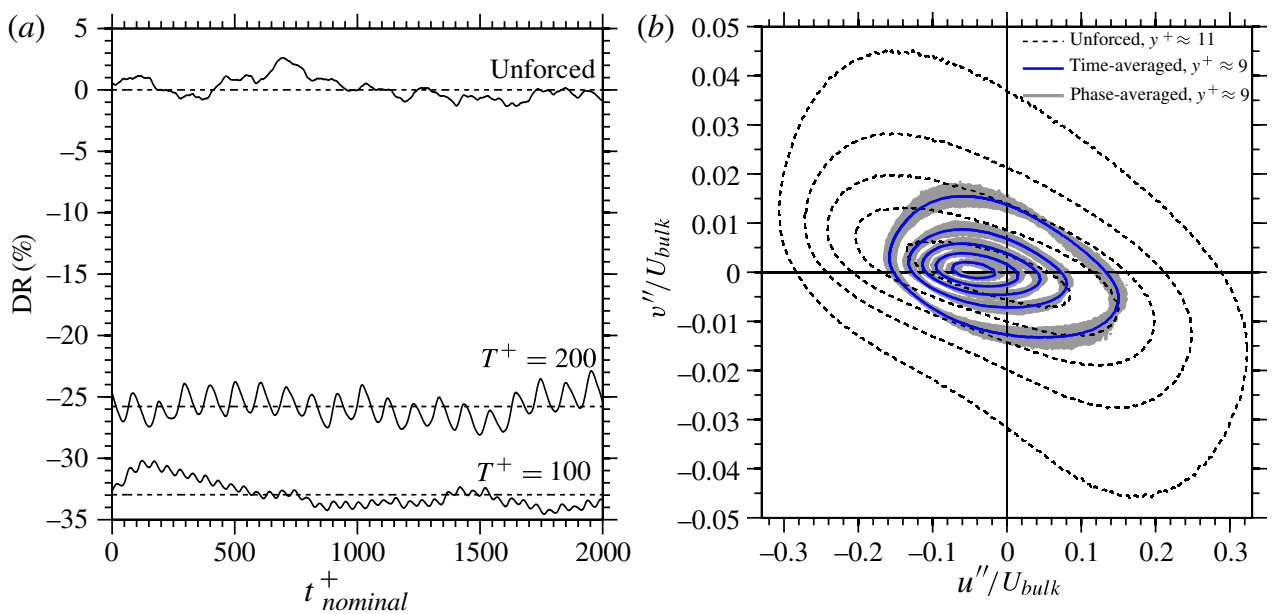

FIGURE 4. (Colour online available at journals.cambridge.org/flm) Sensitivity of the skin friction and turbulent-velocity fluctuations to the forcing phase, $R e_{\tau}=500$ : $(a)$ streamwiseand spanwise-averaged skin friction; $(b)$ phase-averaged PDF $\left(T^{+}=100\right)$.

Support for the above statements is given by figure 4. First, figure 4(a) shows timewise variations of the drag, relative to the time-mean level in the unforced flow, over a period of $2 \times 10^{3}$ viscous time units, following an initial settling-down period of $10^{3}$ units, the time-mean drag being attained, approximately, after three to five actuation cycles. At each instant in time, the drag is derived by averaging the local values in the streamwise and spanwise directions: a sample of 147456 data points. The figure confirms the weak phase dependence of the drag: less than $\pm 1 \%$ of the drag reduction at $T^{+}=100$ and $\pm 2 \%$ at $T^{+}=200$. The relatively long-timescale undulations are due to the effects of large-scale structures in the outer flow. Their presence in figure $4(a)$ reflects the fact that the computational domain only accommodates a few of these structures, so that integration across the wall does not allow a converged (constant) level to be attained. A similar behaviour has been observed and recorded by others (for example Du et al. 2002; Quadrio \& Ricco 2003), with no clear interpretation being given. As we show later, the passage of large outer scales provokes significant skin-friction footprints, and the behaviour shown here may well be a consequence of this physical process. An associated computational issue that may play a role is the streamwise recycling of structures due to the imposition of streamwise periodicity. Although the streamwise domain is around $6 \times 10^{3}$ wall units long, it is possible that large-scale structures, which can be significantly longer than $10^{3}$ wall units, do not fully decorrelate and are then recycled. Second, figure 4(b) shows joint PDF contours for the stochastic fluctuations for the unforced flow and the case $T^{+}=100$. The outermost contours for both cases correspond to a $10 \%$ probability level. For the forced case, the shaded band around the contours (blue solid lines) indicates the PDF phasewise variations. Thus, not only does the drag vary little with phase, but so are the turbulence properties normal to the spanwise direction. Physical implications of plots of time-averaged PDF contours are discussed in $\S 5$.

At $T^{+}=400$ (see figure $3 \mathrm{~b}$ ), the Stokes layer extends well into the buffer region and beyond, and the drag reduction drops to below $10 \%$. The phase-averaged fluctuations in the streamwise velocity, while larger than at $T^{+}=100$, are only of the order of $1 \%$ of the bulk velocity (not shown here). The observation that the increasing intrusion 

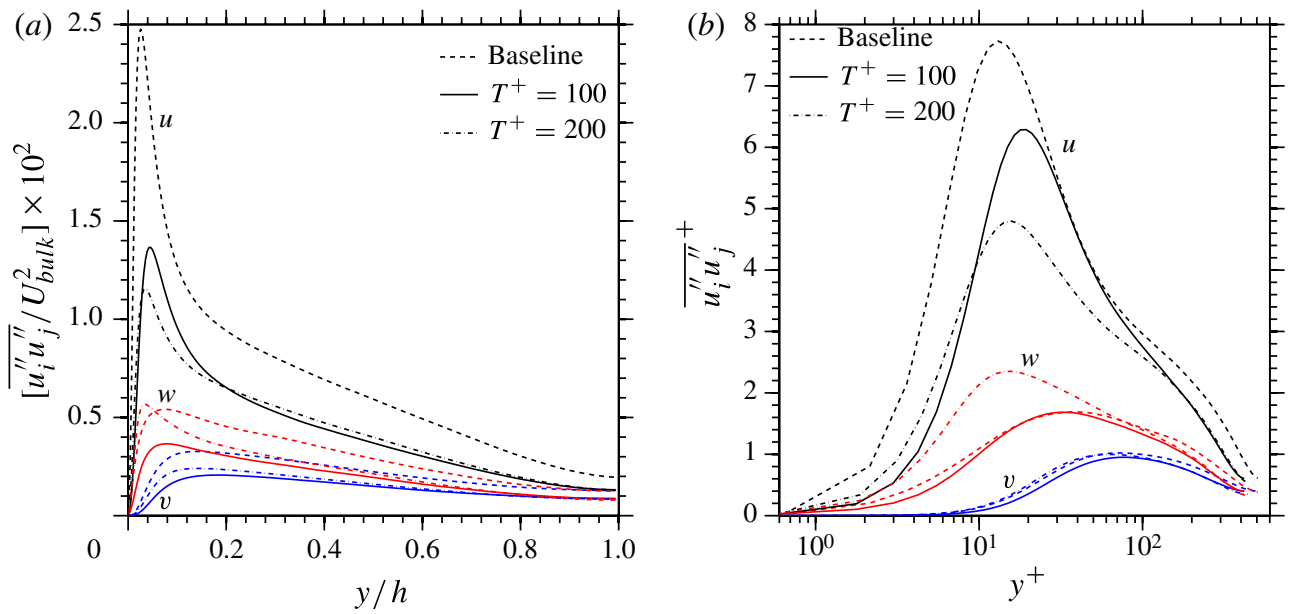

FIGURE 5. (Colour online) Reynolds normal stresses $\left(R e_{\tau}=500\right)$ : $(a)$ outer-scale units; (b) inner-scale units.
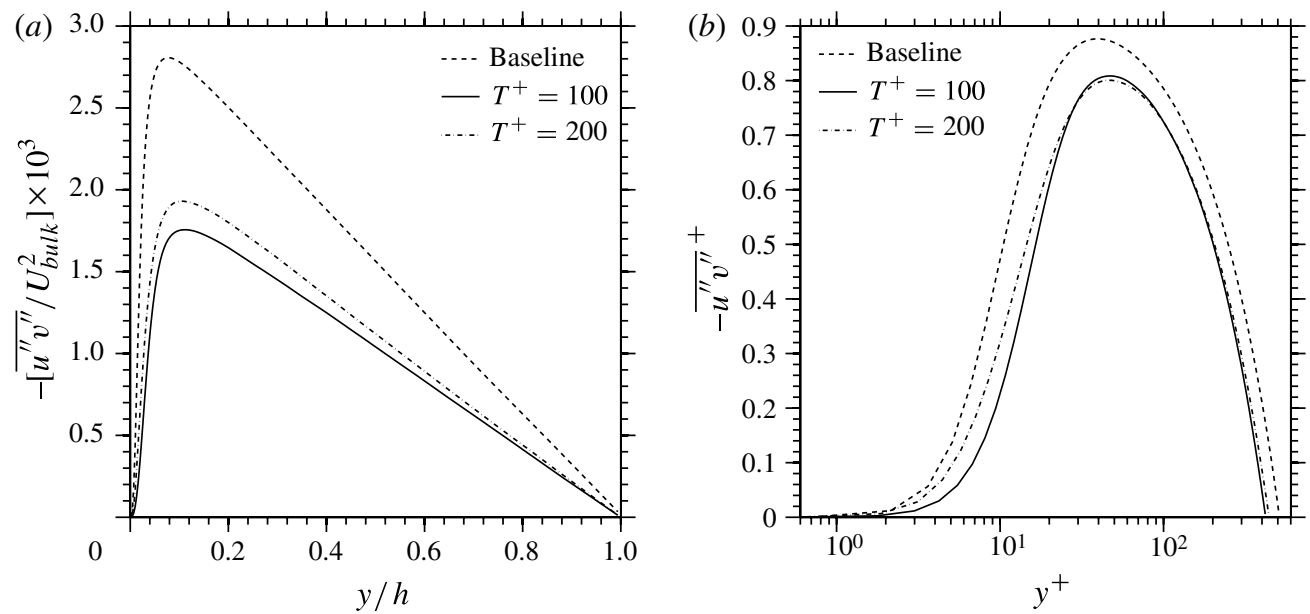

FIGURE 6. Reynolds shear stress $\left(R e_{\tau}=500\right)$ : $(a)$ outer-scale units; $(b)$ inner-scale units.

of the Stokes layer into the turbulent region leads to a substantial degradation in the drag reduction, as $T^{+}$increases well beyond the optimal period and the spanwise shear strain declines, is important and points clearly to properties of the Stokes layer that are critical to the effectiveness of the forcing.

\section{The second moments}

Profiles of stochastic second moments, plotted in terms of outer as well as inner scaling, are shown in figures 5 and 6 . The inclusion of both scaling options is deliberate and rooted in the wish to convey, on the one hand, the response to forcing in absolute terms and, on the other hand, to clarify the degree to which wall scaling procures a universal representation of the properties. Outer scaling also allows changes in the outer portion of the flow to be conveyed with greater clarity. 
The general response to the forcing is a substantial decline, especially close to the wall, of all of the normal stresses and the shear stress, but there are unusual aspects to the relative changes. At the close-to-optimum forcing period, all stresses reduce by at least $30 \%$ throughout the entire channel height. Most important is the decrease in the wall-normal stress, up to $80 \%$ in the viscous sublayer, as this is primarily responsible for the production of the shear stress $\left(\mathscr{P}_{12} \equiv-\overline{v^{\prime \prime} v^{\prime \prime}} \mathrm{d} \bar{u} / \partial y\right)$. Thus, as expected, the shear stress also drops significantly in the viscous sublayer, by around $80 \%$ in absolute terms and $50 \%$ in wall-scaled terms at $y^{+} \sim 10$. Moreover, the maximum turbulent shear-stress drops by around $30 \%$, in absolute terms, consistent with the decline in wall-shear stress of around $32 \%$. As is evident from the innerscaled results, only the profiles for $\overline{v^{\prime \prime} v^{\prime \prime}}$ and $\overline{u^{\prime \prime} v^{\prime \prime}}$ approximately collapse. These two stress components are most closely linked to the wall-shear stress, the former via its decisive contribution to the latter through the production term $\mathscr{P}_{12}$. In the case of the other two components, non-universality is due to complex interactions in the respective budgets and changes to the streak behaviour provoked by the Stokes layer, both discussed later. The fact that the drop in turbulent shear stress in the viscous layer is much larger than in the outer region prevents a collapse of this stress near the wall, as is shown in figure $6(b)$. Thus, with forcing, a much higher proportion of the total shear stress is carried by the viscous component as the turbulent component declines. Moreover, there is a shift in the stress maximum from around $y^{+} \approx 40-48$, indicating the thickening in the viscous sublayer observed earlier by reference to figure 2 .

A curious feature, at first sight, is that the peak streamwise stress near the wall at $T^{+}=200$ is lower than that at 100 . At the same time, the spanwise component at $T^{+}=200$ develops a near-wall maximum that exceeds even the unforced level. Both trends are also observed in the wall-scaled distributions, with the maximum in the spanwise stress at $y^{+}=15$ being especially pronounced.

There are two arguments, one based on statistical and the other on structural observations, that contribute to the explanation of the above seemingly anomalous features. First, as regards the maximum in $\overline{w^{\prime \prime} w^{\prime \prime}}$, it will be shown later that an extra production term, associated with the shear strain of the Stokes motion, is a significant (indeed, major) contributor to the budget of the spanwise stress $\overline{w^{\prime \prime} w^{\prime \prime}}$ at $T^{+}=200$, reaching a maximum at around $y^{+}=12$ and thus elevating that stress. This production is much lower at $T^{+}=100$, because the level of turbulence is very depressed, and does not, therefore, cause a discernible elevation of $\overline{w^{\prime \prime} w^{\prime \prime}}$ at the wall. Second, as will be shown in $\S 10$, the spanwise forcing introduces a periodic realignment of the streaks, and this gives rise to a spanwise component of the streak-oriented fluctuations, and thus to an enhancement of the spanwise stress. This process can also be expected to cause a reduction in the streamwise fluctuations. As the streaks are considerably weaker at $T^{+}=100$ than 200 , the reduction is likely to be proportionately stronger at the higher forcing period, and this goes some way towards explaining the behaviour of the $\overline{u^{\prime \prime} u^{\prime \prime}}$ peak in figure $5(a)$.

The reduction in the separation between the streamwise and spanwise fluctuations is emphasized in figure 7 in terms of distributions of the anisotropy ratios, and this supports the supposition that the periodic re-orientation of the streaks is the principal mechanism responsible for the transfer of energy from $\overline{u^{\prime \prime} u^{\prime \prime}}$ to $\overline{w^{\prime \prime} w^{\prime \prime}}$. While there are no results available for $R e_{\tau}=500$ for forcing periods higher than $T^{+}=200$, figure 8 demonstrates, for $R e_{\tau}=200$, that the diminution of the separation between the streamwise and spanwise fluctuations is progressive as $T^{+}$increases. This further supports the arguments about the role of the streak re-orientation. 

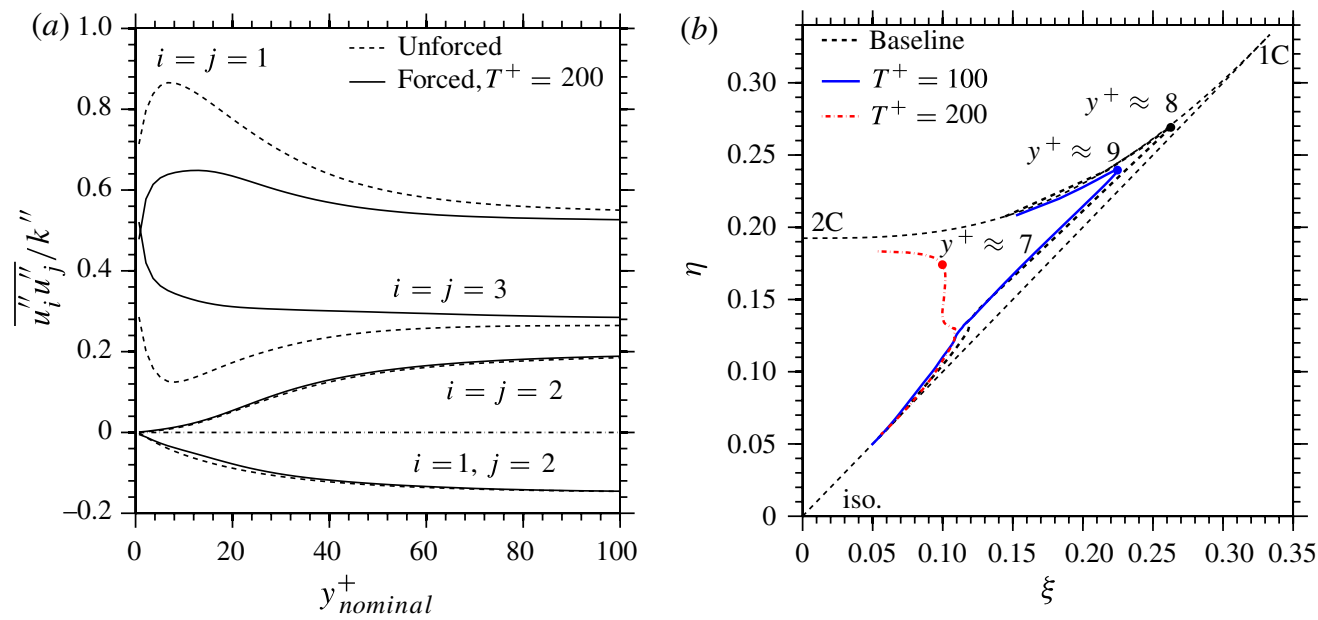

FIGURE 7. (Colour online) Anisotropy at $R e_{\tau}=500$ : (a) Reynolds stresses relative to the turbulence energy $k^{\prime \prime} \equiv \overline{u_{i}^{\prime \prime} u_{i}^{\prime \prime}}$; (b) Lumley triangle for the stochastic fluctuations, $b_{i j}^{\prime \prime} \equiv$ $\overline{u_{i}^{\prime \prime} u_{j}^{\prime \prime}} / \overline{u_{k}^{\prime \prime} u_{k}^{\prime \prime}}-\delta_{i j} / 3$ and $6 \eta^{2} \equiv b_{i j}^{\prime \prime} b_{j i}^{\prime \prime}, 6 \xi^{3} \equiv b_{i j}^{\prime \prime} b_{j k}^{\prime \prime} b_{k i}^{\prime \prime}$.
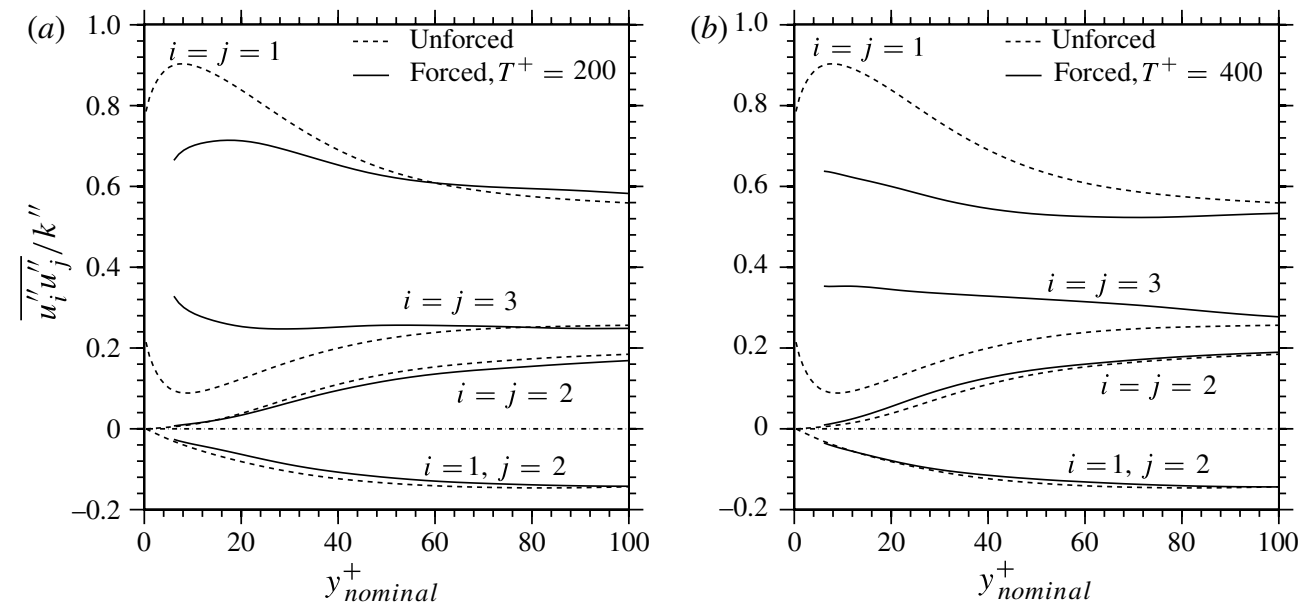

FIGURE 8. Reynolds stresses relative to the turbulence energy $k^{\prime \prime} \equiv \overline{u_{i}^{\prime \prime} u_{i}^{\prime \prime}}\left(\operatorname{Re}_{\tau}=200\right)$ : (a) $T^{+}=200 ;\left(\right.$ b) $T^{+}=400$.

Figure 7 provides a supplementary view of the impact of forcing on the state of anisotropy, by way of the so-called 'Lumley map'. This shows variations of the second and third anisotropy invariants along wall-normal lines traversing the flow from the wall to the channel centreline. Forcing is seen to cause a progressive weakening of the distinctive peak that characterizes the trend towards the one-component state that is normally observed close to the wall prior to the asymptotic approach to the two-component state at the wall itself. The virtual suppression of this peak at $T^{+}=200$, and the fact that the route for this period deviates from that pertaining to the log-law portion adjoining the buffer layer (along the lower line of the triangle), suggests that the isotropization effect arises from the progressive penetration of the 

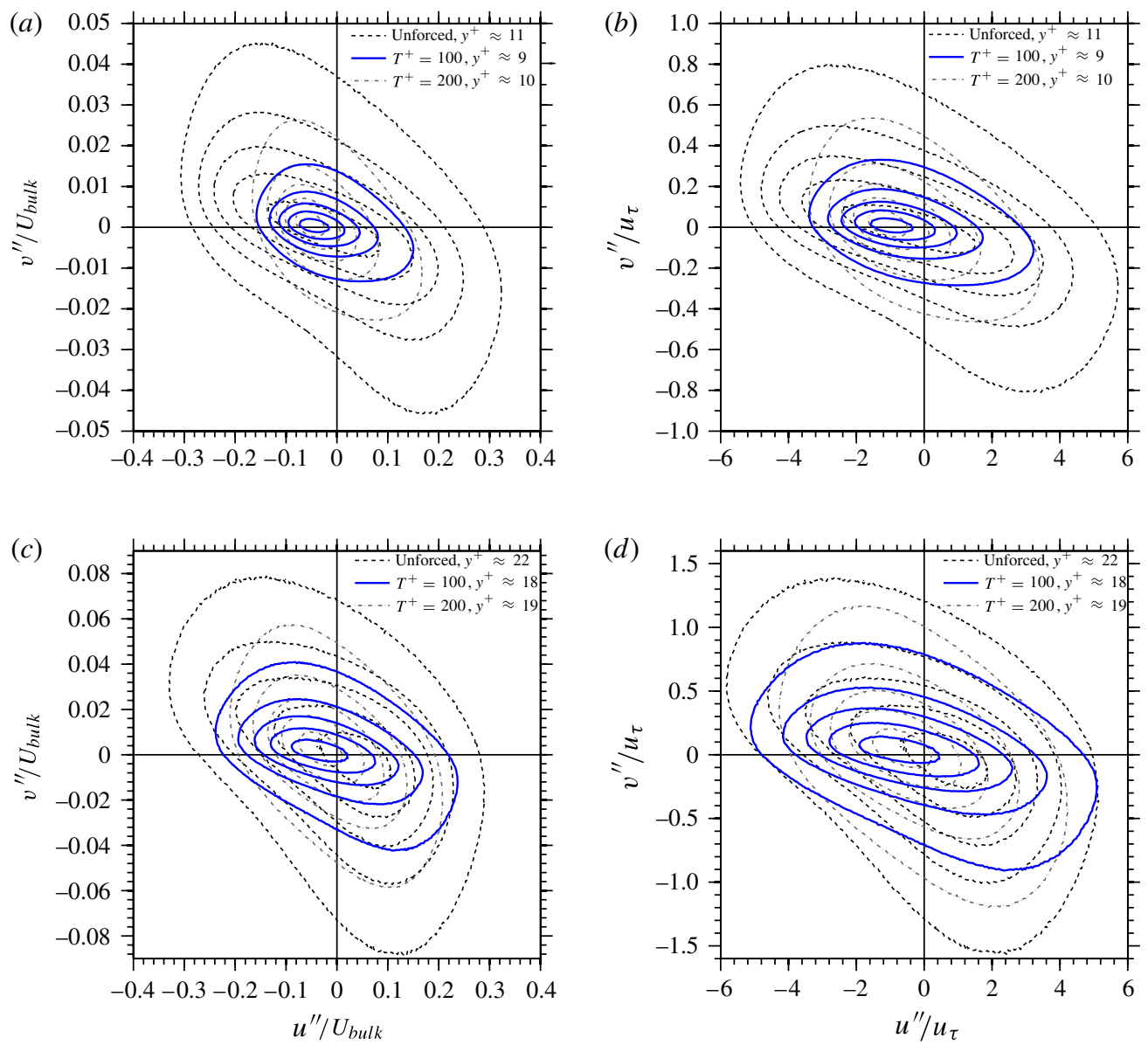

FIGURE 9. (Colour online) Outer-scaled and inner-scaled PDFs for $\left(u^{\prime \prime}, v^{\prime \prime}\right)$ for $R e_{\tau}=500$ at $y+\approx 11$ and 22 . Contours give lines of $10,30,50,70$ and $90 \%$ of the maximum probability level: $(a)$ outer scaling; $(b)$ inner scaling; $(c)$ outer scaling; $(d)$ inner scaling.

Stokes layer into the flow, thus diminishing the damping effects of the forcing on the streaks and enhancing the influence of the periodic realignment of the streaks on the normal-stress separation. As an interesting aside, attention is drawn to recent work by Frohnapfel, Hasegawa \& Kasagi (2010), in which it is argued that one promising route to drag reduction is through the damping of spanwise fluctuations, a process that drives turbulence towards a state of one-component turbulence in the Lumley triangle. In fact, Frohnapfel et al. (2007) argue that there is a more general relationship between drag reduction and an enhanced trend towards one-component turbulence at the wall. This is contradicted by the present observations: the flow here becomes more isotropic, and spanwise fluctuations are amplified by the forcing, yet the drag is reduced.

\section{Probability density functions}

Joint PDFs at $y^{+}=11$ and 22 are shown in figure 9 and allow a view to be gained of which fluctuations are preferentially damped, how the correlation between 

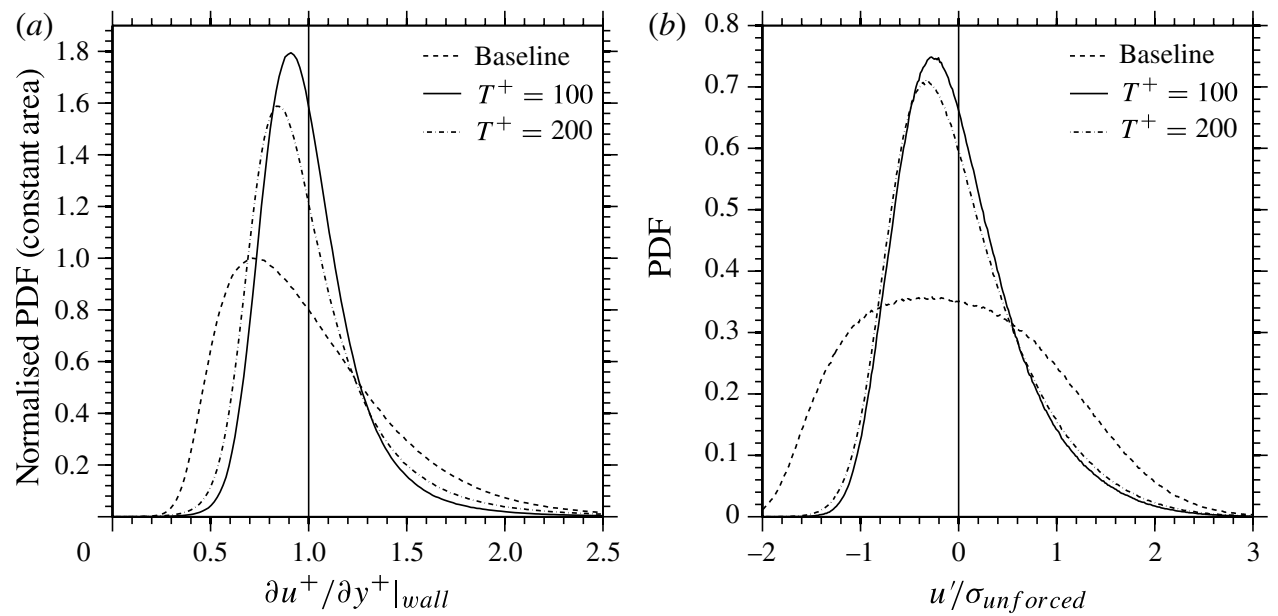

FIGURE 10. PDFs of $\partial u^{+} /\left.\partial y^{+}\right|_{\text {wall }}$ and $u^{\prime}$ at $y^{+} \approx 10$.

streamwise and wall-normal fluctuations is affected, and whether this correlation is asymmetrically modified when associated with sweeps and ejections. The slight differences in $y^{+}$among the sets of contours in any one of the plots are due to the fact that data have been taken from grid planes at constant heights $y / h$. The PDFs are normalized with outer as well as inner scales. The former is intended to bring out the forcing-induced damping in absolute terms, while the latter conveys the fact that the near-wall turbulent fluctuations are depressed much below the level that would make the turbulence properties collapse with inner scaling: a point already made in relation to the second moments (figures 5 and 6). The importance of this depression to the drag-reduction margin is well delineated by the identity from Fukagata, Iwamoto \& Kasagi (2002), which shows that the turbulent shear stress very close to the wall is disproportionately influential to the skin friction. However, at wall distances into the buffer layer and beyond (the latter not included in figure 9), inner scaling is found to progressively improve the collapse of the PDF contours. In fact, a full collapse is virtually attained at $y^{+}>300$.

Apart from the much tighter PDF contours for the forced flow, signifying strong damping, two features deserve to be highlighted, specifically in relation to $T^{+}=100$. First, the orientation of the contours (or rather of their major axes) for the forced flow is tilted towards the horizontal, relative to the baseline flow. This reorientation points to a combination of a disproportionate reduction of wall-normal fluctuations, relative to the streamwise components, and a lower correlation between $u^{\prime \prime}$ and $v^{\prime \prime}$ fluctuations, both implying a drop in the shear stress. Second, there is an indication of a preferential decline in the contributions to the shear stress in the second quadrant, characterized by negative streamwise fluctuations and positive wall-normal fluctuations, i.e. ejections. The implication is that the contribution of sweep events, associated with positive streamwise fluctuations, increases in relative terms.

It is reasonable to expect that this bias, specifically in the PDF closest to the wall, $y^{+} \approx 11$, would be reflected by a corresponding asymmetry in the fluctuations of the wall shear stress. That this is indeed the case as demonstrated in figure 10, which shows the PDF of the inner-scaled wall shear strain alongside the PDF of the streamwise-velocity fluctuations at $y^{+} \approx 10$. Attention is drawn to the manner in which these PDFs have been constructed. The area under the PDFs in figure 10(a) is constant, 
having been derived from the PDF of the unforced flow, which is normalized to have a peak value of 1 . In figure 10(b), the area under all PDFs is also constant, but equal to 1, and the widths of the PDFs have been normalized by the variance of the PDF for the unforced flow. As expected, forcing diminishes the range of gradient fluctuations, but the magnitude of the reduction is biased towards the low-speed fluctuations. Although high-speed fluctuations also diminish, this diminution is less pronounced, and the tail of the highest-speed events, associated with strong sweeping motions, persists.

While figure 9 shows that the velocity fluctuations are also materially depressed at $T^{+}=200$, some of the features described about for $T^{+}=100$ are less pronounced, in particular the tilting of the major axes towards the horizontal. However, there continues to be a noticeable bias in probability towards high-speed fluctuations in the viscous sublayer, also seen in figure 10. The striking differences in shape and orientation between the contour sets at $T^{+}=100$ and 200 is partly linked to the stronger diminution, at the higher forcing period, of the streamwise component, as is clearly observed in figure 5 and discussed by reference to streak tilting in relation to that figure.

\section{Second-moment budgets}

This section presents second-moment budgets for $R e_{\tau}=500$. Corresponding budgets for $R e_{\tau}=200$ show analogous qualitative behaviour. For this lower value, $T^{+}=400$ has also been examined, and the budgets were observed to be close to those for the unforced case, reflecting the rapidly weakening (indeed, in some respects, counterproductive) effects of forcing at long actuation periods on the skin friction.

The budgets are expressed by the exact transport equations for the stochastic Reynolds stresses, and these are as follows (see Appendices B and C for details):

$$
\begin{aligned}
& \frac{\mathrm{d} \overline{u^{\prime \prime} u^{\prime \prime} v^{\prime \prime}}}{\mathrm{d} y}=-2 \overline{u^{\prime \prime} \frac{\partial p^{\prime \prime}}{\partial x}}-2 \overline{u^{\prime \prime} v^{\prime \prime}} \frac{\mathrm{d} \bar{u}}{\mathrm{~d} y} \underbrace{-\overline{2\left\langle u^{\prime \prime} v^{\prime \prime}\right\rangle \frac{\partial \tilde{u}}{\partial y}}}_{\widetilde{\mathscr{P}}_{11}} \\
& -\frac{2}{R e} \sum_{i=1}^{3} \overline{\left(\frac{\partial u^{\prime \prime}}{\partial x_{i}}\right)^{2}}+\frac{1}{R e} \frac{\mathrm{d}^{2} \overline{u^{\prime \prime} u^{\prime \prime}}}{\mathrm{d} y^{2}}, \\
& \frac{\mathrm{d} \overline{v^{\prime \prime} v^{\prime \prime} v^{\prime \prime}}}{\mathrm{d} y}=-2 \overline{v^{\prime \prime} \frac{\partial p^{\prime \prime}}{\partial y}}-\frac{2}{R e} \sum_{i=1}^{3} \overline{\left(\frac{\partial v^{\prime \prime}}{\partial x_{i}}\right)^{2}}+\frac{1}{R e} \frac{\mathrm{d}^{2} \overline{v^{\prime \prime} v^{\prime \prime}}}{\mathrm{d} y^{2}},
\end{aligned}
$$

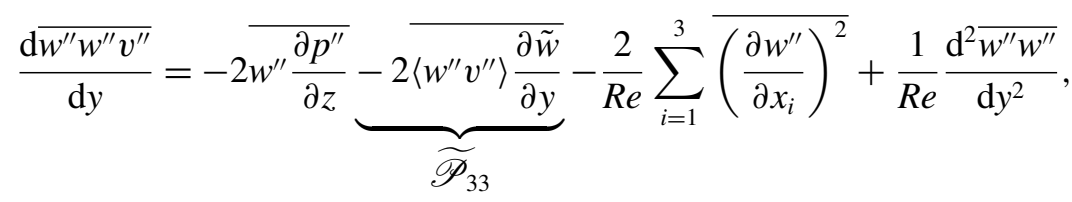

$$
\begin{aligned}
& \frac{\mathrm{d} \overline{u^{\prime \prime} v^{\prime \prime} v^{\prime \prime}}}{\mathrm{d} y}=-\overline{u^{\prime \prime} \frac{\partial p^{\prime \prime}}{\partial y}}-\overline{v^{\prime \prime} \frac{\partial p^{\prime \prime}}{\partial x}}-\overline{v^{\prime \prime} v^{\prime \prime}} \frac{\mathrm{d} \bar{u}}{\mathrm{~d} y} \underbrace{-\overline{\left\langle v^{\prime \prime} v^{\prime \prime}\right\rangle \frac{\partial \tilde{u}}{\partial y}}}_{\widetilde{\mathscr{P}}_{12}} \\
& -\frac{2}{R e} \sum_{i=1}^{3} \overline{\frac{\partial u^{\prime \prime}}{\partial x_{i}} \frac{\partial v^{\prime \prime}}{\partial x_{i}}}+\frac{1}{R e} \frac{\mathrm{d}^{2} \overline{u^{\prime \prime} v^{\prime \prime}}}{\mathrm{d} y^{2}} \text {. }
\end{aligned}
$$



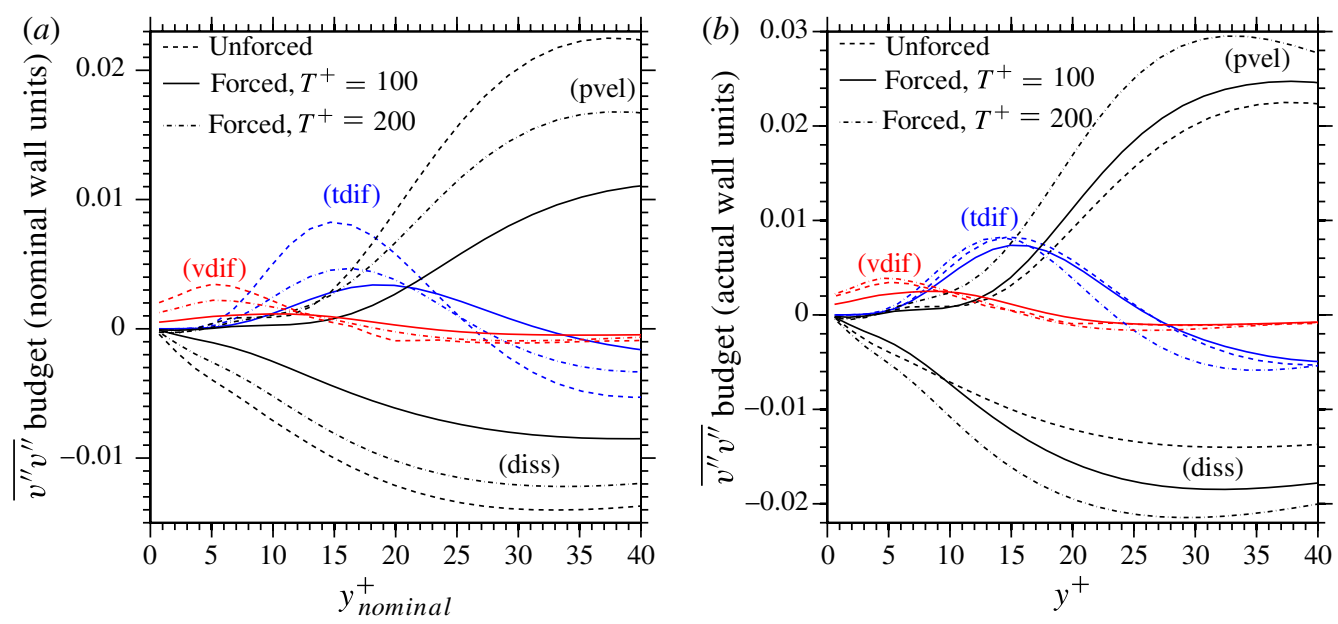

FIGURE 11. Budget of $(7.1 b)$ for $R e_{\tau}=500$ with (tdif) turbulent diffusion, (pvel) pressure-velocity, (diss) dissipation and (vdif) viscous diffusion: (a) nominal units; (b) actual units.

Particular attention is drawn to the production fragments $\widetilde{\mathscr{P}}_{i j}$, which account for the interaction between phase-averaged stresses and gradients of the phase-velocity fluctuations. Except for $\widetilde{\mathscr{P}}_{33}, \widetilde{\mathscr{P}}_{i j}$ terms are found to be three orders of magnitude smaller than their respective mean-flow production terms $\mathscr{P}_{i j}$, and they are therefore negligible. The equation for $\overline{v^{\prime \prime} v^{\prime \prime}}$ distinguishes itself by the absence of explicit terms involving any fluctuations other than $v^{\prime \prime}$ itself. Hence, importantly, any effects of the forcing on this stress must be rooted entirely in the linkage to other equations via the pressure-velocity term.

A frequently adopted practice is to split the pressure-velocity interaction into pressure-strain and pressure-diffusion fragments, the former trace-free and the latter purely redistributive within any one of the budgets. This fragmentation is not effected herein, but its examination has revealed, particularly in the case of $\overline{v^{\prime \prime} v^{\prime \prime}}$, that the two parts are of opposite sign, each much larger than the sum, especially near the wall, where the two parts virtually cancel each other. This behaviour has been observed before (e.g. Fröhlich et al. 2005), and the fragmentation is thus regarded as unhelpful for the present discussion.

The budgets of greatest interest are those for $\overline{v^{\prime \prime} v^{\prime \prime}}$ and $\overline{u^{\prime \prime} v^{\prime \prime}}$, figures 11 and 12 , the former dictating the production of the shear stress and the latter linked directly to the friction drag, as well as governing the production of the streamwise stress and the turbulence energy. The budgets are deliberately scaled in two ways: one based on the nominal, unforced, wall-shear stress and the other on the actual value. The former scaling of interest, as it conveys, essentially in absolute terms, the forcing-induced responses of the various budget contributions to forcing, relative to the unforced state.

The budget for $\overline{v^{\prime \prime} v^{\prime \prime}}$ is dominated by a balance between the pressure-velocity process, feeding energy into this stress, and dissipation. Forcing is seen to reduce the pressure-velocity term, and this is accompanied by a reduction in the dissipation. An important observation relates to the interplay between the pressure-velocity and the turbulent-transport (diffusion) terms. The former is large outside the viscous sublayer, but halved upon forcing at $T^{+}=100$. Within the viscous sublayer, the 

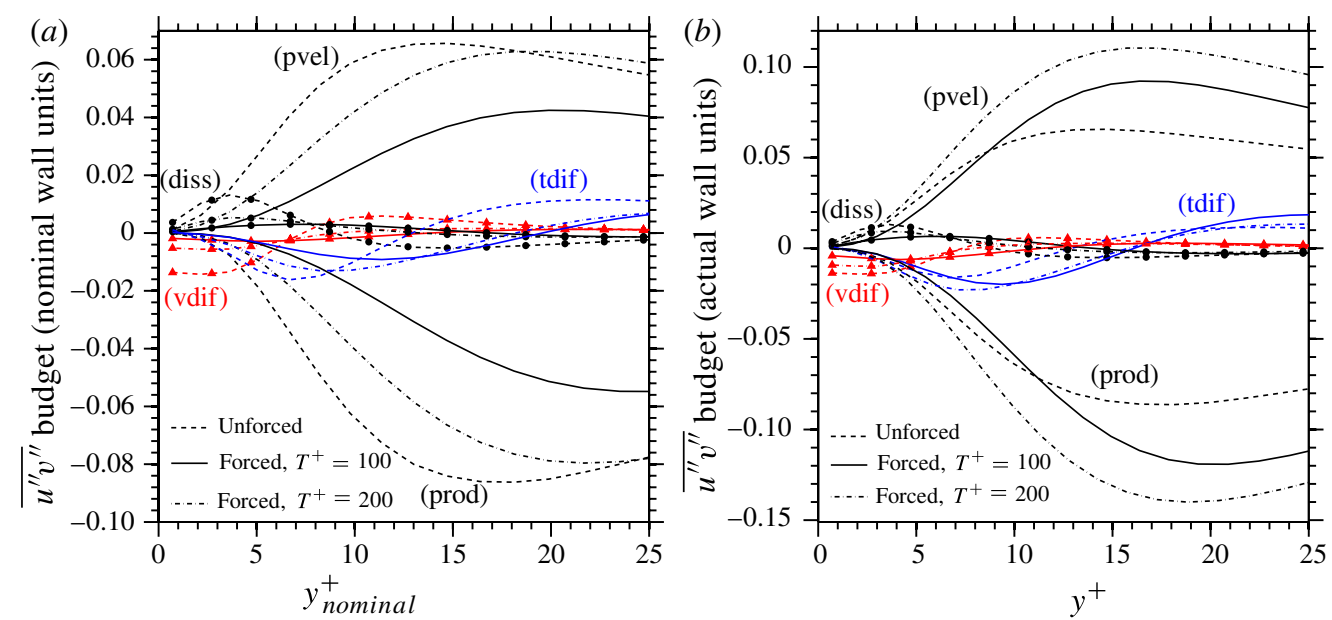

FIGURE 12. Budget of $(7.1 d)$ for $R e_{\tau}=500$ with (tdif) turbulent diffusion, (pvel) pressure-velocity, (prod) production (diss) dissipation and (vdif) viscous diffusion. $\widetilde{\mathscr{P}}_{12} \sim$ $O\left(10^{-5}\right)$ (at most) and is not reproduced: $(a)$ nominal units; $(b)$ actual units.

pressure-velocity term is a minor contributor, but there is, nevertheless, a noteworthy decline in this term, especially at $T^{+}=100(\sim 75 \%$ relative to the unforced case). The reduction in the pressure-velocity term in the outer region is then accompanied by a decline in the turbulent diffusion, the role of which is to transport the excess of $v^{\prime \prime} v^{\prime \prime}$ in the outer region towards the viscous sublayer. In the unforced case, this is the most important process by which energy is fed into $\overline{v^{\prime \prime} v^{\prime \prime}}$ from the outer region towards the wall. Very close to the wall, viscous diffusion takes over this role. In the presence of forcing, the strong decrease in the pressure-velocity interaction also depresses the diffusion process and hence the near-wall level of $\overline{v^{\prime \prime} v^{\prime \prime}}$.

Identifying the fundamental origin of the depression in the pressure-velocity term in the $\overline{v^{\prime \prime} v^{\prime \prime}}$ budget is, arguably, central to the drag-reduction scenario. It is tempting to speculate that the strong (unsteady) shearing associated with the Stokes motion shields the near-wall region from eddies penetrating from the outer region, thereby reducing the wall-normal fluctuating-pressure gradient and thus depressing the pressure-velocity interaction. This is a suggestion made previously by Ricco (2004), based simply on the observation that sweeping motions are depressed by the oscillatory forcing. Some light on this question is shed by figure 13(a), in which the root-mean-squared (r.m.s.) values of the gradients of the pressure fluctuations are plotted. The expectation is that a reduced level of the r.m.s. value is associated with a diminution in the pressure-velocity correlation over and above that resulting from the reduction in the velocity fluctuations alone. Of greatest interest is the distribution for $\overline{\left[\partial p^{\prime \prime} / \partial y\right]^{2}}(i=2$ in the figure), pertaining to $\overline{v^{\prime \prime} v^{\prime \prime}}$. The most striking feature is the reduction of the wall-normal r.m.s. value at $T^{+}=100$ in the region extending from the wall to $y^{+} \sim 75$. Somewhat surprisingly, the reduction at $T^{+}=200$ is modest, and also confined to the region beyond $y^{+} \sim 20$. Consistently, however, the pressure-velocity term at $T^{+}=200$ is only marginally lower than that of the unforced case below about $y^{+}=20$, as can be observed in figure 11. Hence, figure 13 provides some support for the conclusion that the sensitivity of the pressure-velocity process in $\overline{v^{\prime \prime} v^{\prime \prime}}$ to forcing occurs partly via a 

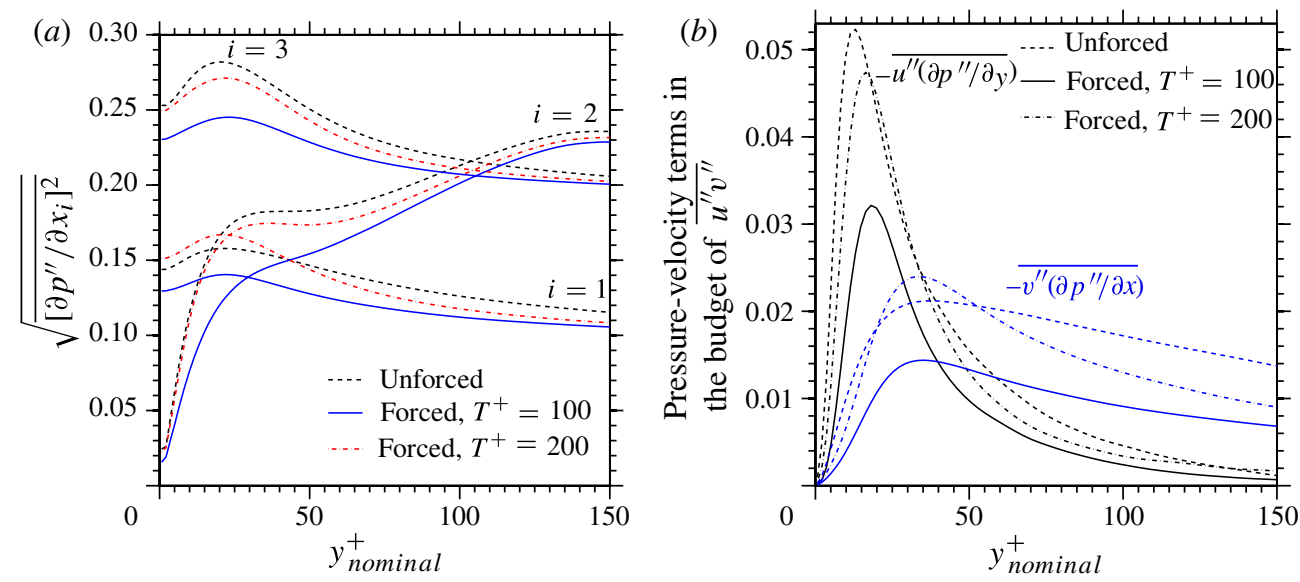

FIGURE 13. (Colour online) Wall-normal profiles of the standard deviation of the fluctuating pressure gradients and decomposition of the pressure-velocity term in $(7.1 d)$ in its constituents $\left(R e_{\tau}=500\right)$.

reduction in pressure-gradient fluctuations beyond the immediate wall region, which is then accompanied by a decline in the turbulent transport of $\overline{v^{\prime \prime} v^{\prime \prime}}$ towards the wall.

The $\overline{v^{\prime \prime} v^{\prime \prime}}$ budget, when scaled with the actual wall-shear stress, is interesting in so far as it shows that the respective contributions almost collapse, suggesting a close relationship between the processes affecting that stress and the wall-shear stress. This is not unexpected, as the shear stress is closely linked to $\overline{v^{\prime \prime} v^{\prime \prime}}$, via the production of the former. Moreover, all processes, but the pressure-velocity interaction, depend only upon $\overline{v^{\prime \prime} v^{\prime \prime}}$.

The budget for the shear stress, figure 12, is dominated by the production and the velocity-pressure terms, the latter reducing the shear-stress magnitude. As expected, in view of the role of $\overline{v^{\prime \prime} v^{\prime \prime}}$, the production drops drastically, by up to $80 \%$, in the viscous sublayer at $T^{+}=100$. Correspondingly, the pressure-velocity term declines, with the difference being absorbed by relatively low turbulent diffusion away from the wall and viscous diffusion very close to the wall. Dissipation is negligible outside the viscous sublayer, rising only very close to the wall, a behaviour consistent with anisotropy in the smallest scales. An evaluation of the two contributions to the pressure-velocity process in the shear-stress budget is provided in figure $13(b)$, and this shows that the reduction in this process in the viscous layer is primarily due to the interaction of $u^{\prime \prime}$ and the wall-normal pressure-fluctuation gradient, the latter also featuring in the equation for $\overline{v^{\prime \prime} v^{\prime \prime}}$. As already shown in figure 13(a), the wall-normal pressure-fluctuation gradient is substantially reduced by the forcing. The maximum reduction in the r.m.s. value of $u^{\prime \prime}$ in the viscous layer at $T^{+}=100$ is $84 \%$ (at $y^{+} \approx 2$, in nominal units), and this further adds to the diminution of the pressure-velocity term in the presence of forcing. Figure 12 shows that the use of actual wall scaling tends to steer the budgets towards a collapse, although this trend is mostly qualitative and less clear-cut than for the budget of $\overline{v^{\prime \prime} v^{\prime \prime}}$ with which the shear stress is linked via the shear-stress production. In this context attention is drawn to figure 6 and the associated discussion, which highlights the lack of universality of the wall-scaled shear stress itself near the wall.

The budget for $\overline{u^{\prime \prime} u^{\prime \prime}}$ is shown in figure 14. This shares many features with those observed in the budget for the turbulence energy (the most important difference being 

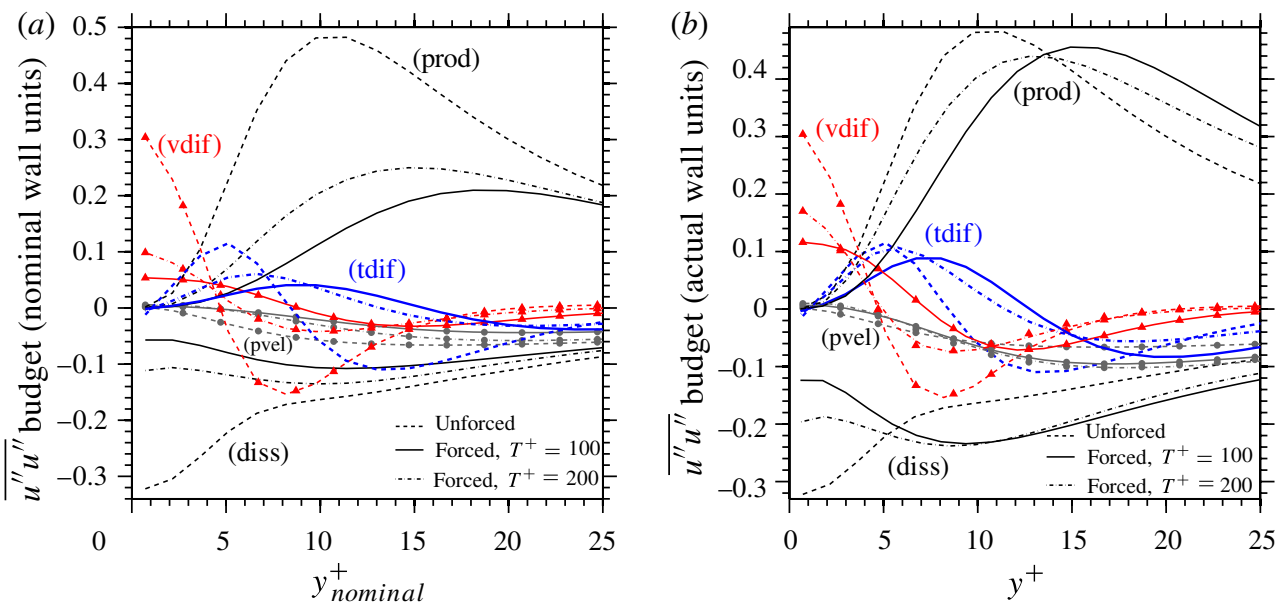

FIGURE 14. Budget of (7.1a) for $R e_{\tau}=500$ with (tdif) turbulent diffusion, (pvel) pressure-velocity, (prod) production, (diss) dissipation and (vdif) viscous diffusion. $\widetilde{\mathscr{P}}_{11} \sim$ $O\left(10^{-3}\right)$ and is not reproduced: $(a)$ nominal units; $(b)$ actual units.

in respect of the pressure-velocity interaction), which is the reason for not including the latter herein. Two processes to note first are the major decline in production and the somewhat less drastic decrease in dissipation. Without forcing, diffusive transport makes an important contribution to the balance, both draining energy beyond $y^{+} \sim 5$, counteracting production, and transporting it towards the wall, with dissipation balancing this transport. The strong forcing-induced decline in production causes the diffusive transport towards the wall to be diminished and thus also the near-wall dissipation. The pressure-velocity term is relatively minor, extracting energy from $\overline{u^{\prime \prime} u^{\prime \prime}}$ and diverting it to the other two normal stresses. Again, the forcing leads to a decline in this process, the level of reduction being, typically, 70-90\% close to the wall, relative to the baseline flow.

An observation that is of relevance to the non-monotonic behaviour (in $T^{+}$) in the peak streamwise stress (figure 5), is that the production levels at $T^{+}=100$ and 200 do not differ dramatically around $y^{+}=15-20$, the position at which the maximum of $\overline{u^{\prime \prime} u^{\prime \prime}}$ occur in the presence of forcing. Similarly, the differences in the respective diffusion and pressure-velocity contributions are small, but it is nevertheless noteworthy that turbulent diffusion at $T^{+}=200$ is positive and lower than that at $T^{+}=100$, tending to reduce $\overline{u^{\prime \prime} u^{\prime \prime}}$ in this $y^{+}$range at the longer forcing period. These subtle changes in the balance make it difficult to correlate, unambiguously, particular features in the budgets with the behaviour of the maximum of $\overline{u^{\prime \prime} u^{\prime \prime}}$ in figure 5. What emerges clearly, however, is the substantial decline in production, via $\overline{u^{\prime \prime} v^{\prime \prime}}$, in the region $y^{+}=5-15$, which is the major driver of the reduced level of this normal stress, and this then causes wall-normal diffusive transport to decline, with dissipation following suit.

In accordance with expectation, most contributions in the wall-scaled $\overline{u^{\prime \prime} u^{\prime \prime}}$ budget (figure 14b) do not collapse. An exception, qualitatively, is the production, and this reflects the close link to the shear stress. However, the other terms (diffusion, in particular) do not scale in any obvious sense with the wall shear stress. As the near-wall dissipation closely follows viscous diffusion, the former does not collapse either, at least not close to the wall. 

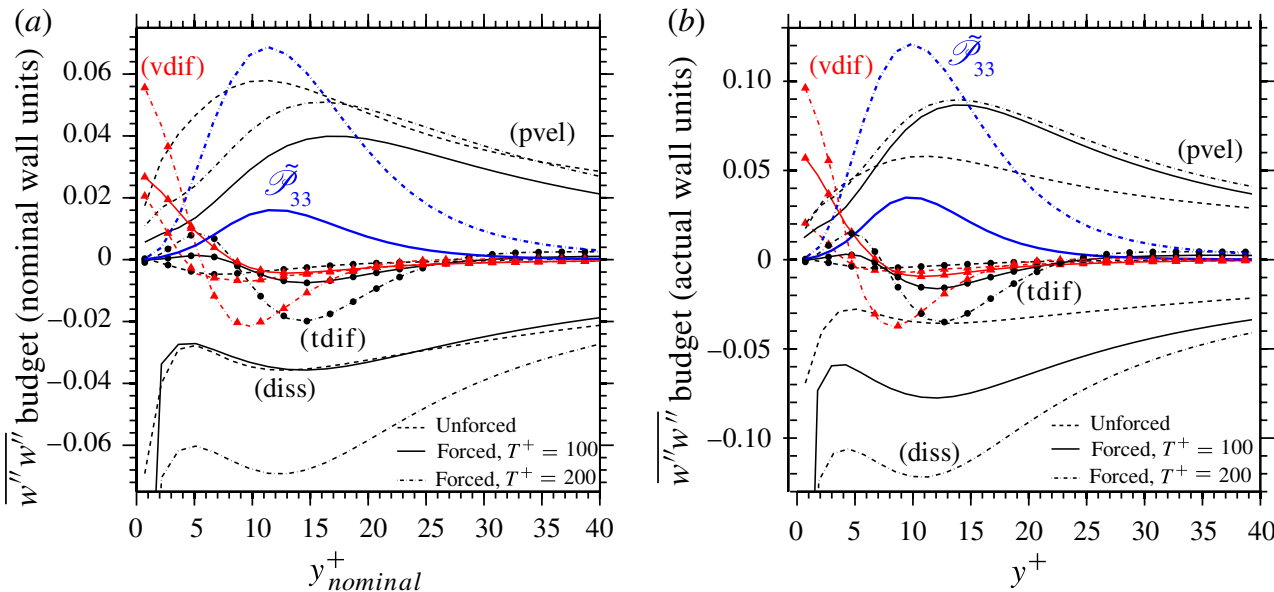

FIGURE 15. Budget of (7.1c) for $R e_{\tau}=500$ with (tdif) turbulent diffusion, (pvel) pressure-velocity, (diss) dissipation and (vdif) viscous diffusion: $(a)$ nominal units; $(b)$ actual units.

The budget for $\overline{w^{\prime \prime} w^{\prime \prime}}$ is shown in figure 15. Its most interesting feature is the large production due to the Stokes motion. This is especially high at $T^{+}=200$, as a consequence of the combination of higher Stokes straining and $\left\langle w^{\prime \prime} v^{\prime \prime}\right\rangle$ levels in the upper part of the viscous layer, the latter reflecting the elevation of $w^{\prime \prime}$ by the Stokes strain. This forcing-related production is responsible for the peak in $\overline{w^{\prime \prime} w^{\prime \prime}}$, seen in figure 5. It will also be shown later, by reference to visualizations of streak-conditional phase-averaged data, how the periodic forcing generates peaks in $\overline{w^{\prime \prime} w^{\prime \prime}}$ when the gradient of the Stokes motion periodically reaches its maximum. Predictably, the response of this production is a substantial elevation in the diffusion process, with the consequent transfer of $\overline{w^{\prime \prime} w^{\prime \prime}}$ from the high-production region towards the wall, and in the dissipation. As the production diminishes strongly at $T^{+}=100$, all terms decline in the budget. Surprisingly, the pressure-velocity contribution declines monotonically with forcing, despite the increased production, with increased dissipation securing the balance. The fact that the velocity-pressure term involves $w^{\prime \prime}$ suggests that the decline in this term, especially at $T^{+}=200$, may be due to an attenuation in the spanwise gradient of the pressure fluctuations. However, figure 13 does not provide support for this supposition, and this suggests a weakening correlation between spanwise velocity fluctuations and the spanwise gradient of pressure fluctuations as the forcing period is decreased. As expected, figure 15 shows that scaling with the actual wall-shear stress does not result in a collapse of the budget. Such a collapse can simply not occur, because $\widetilde{\mathscr{P}}_{33}$ is a major constituent of the budget when forcing is effective, and this is not linked in any direct manner to the wall shear stress.

\section{Enstrophy}

Instantaneous and conditionally averaged cross-flow fields, such as those shown in figures 16 and 17, and discussed in detail in $\S 9$, suggest, in common with received wisdom, that streaks are associated with quasi-streamwise vortices. Hence, the expectation is that forcing would result in a decline in streamwise enstrophy, commensurate with the attenuation of wall-normal fluctuations. This relationship is investigated by reference to figures 18 and 19. The former compares the phase- 

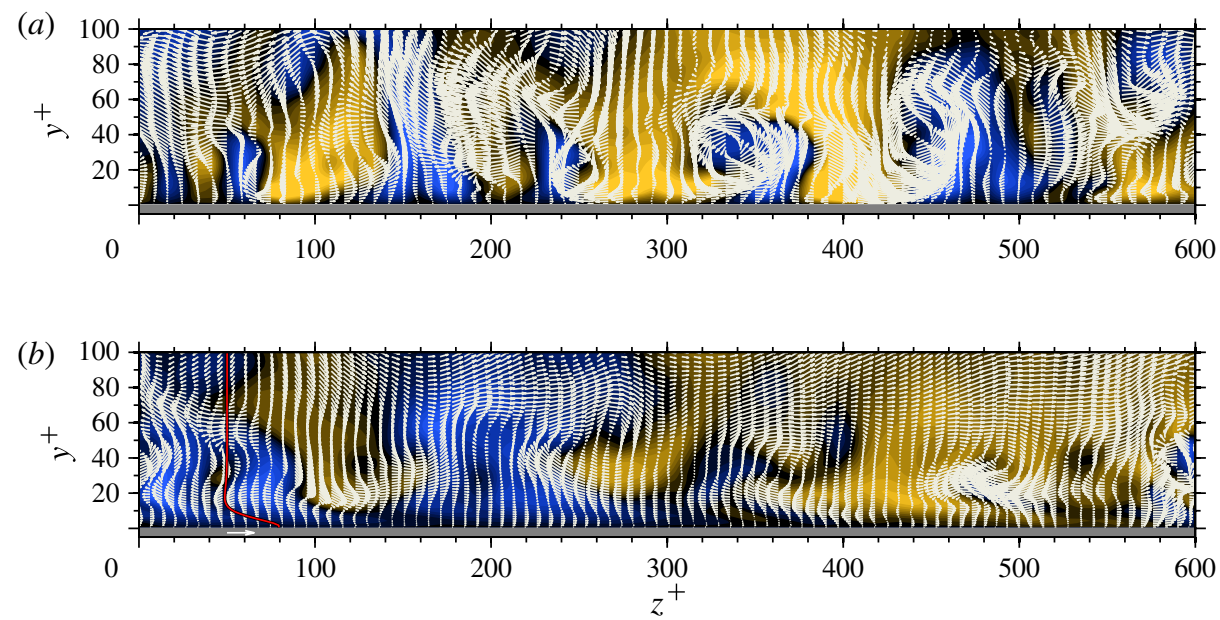

FIGURE 16. Transverse-flow cross-section of the channel showing the stochastic part of the velocity-vector fluctuations at $R e_{\tau}=500$. The colour contours give the streamwise component clipped to $\left[-0.25 U_{\text {bulk }}, 0.25 U_{\text {bulk }}\right]$ (blue is for negative, yellow for positive). The superimposed vector field represents the spanwise and wall-normal components using the exact same scaling factor in both $(a)$ and $(b)$. In $(b)$, the red profile gives the phase-averaged spanwise velocity profile at this particular phase. The white arrow indicates the plate velocity at this particular instant: $(a)$ forcing OFF; $(b)$ forcing ON, $T^{+}=100$.
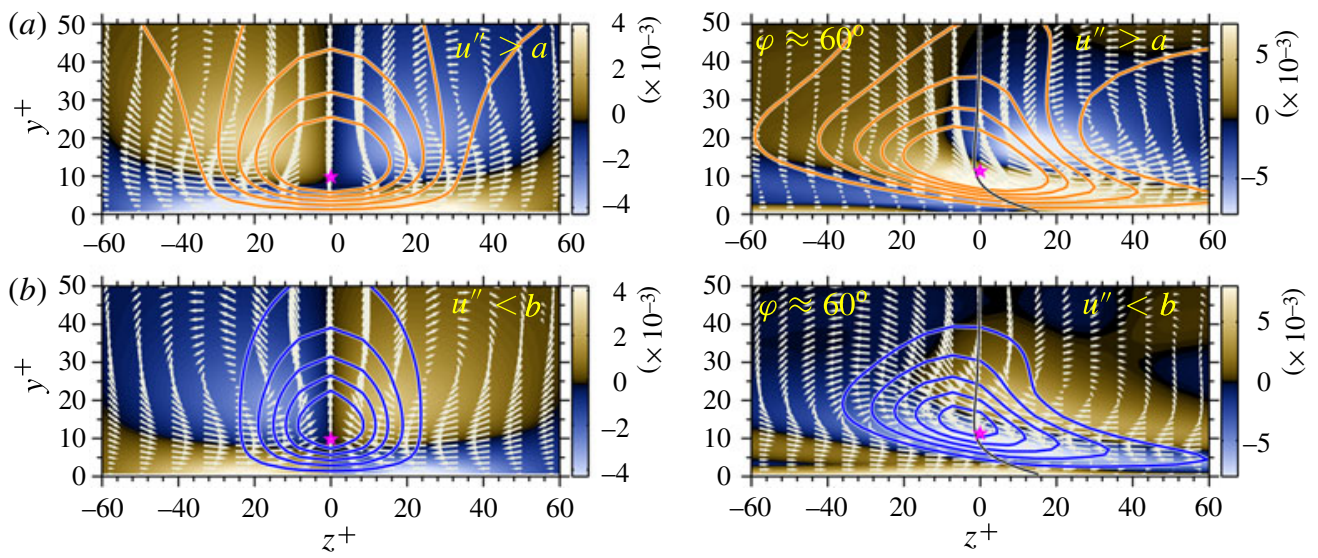

FIGURE 17. Conditionally averaged streaks obtained by sampling the flow, after application of a high-pass filter to remove super-streak modulations, such that $u^{\prime \prime}<b$ and $u^{\prime \prime}>a$ at $y^{+} \approx 10$ (magenta star). Contour lines give the streamwise velocity contours $u^{\prime \prime} / u_{\tau} \in$ $\{-2.5,-2,-1.5,-1,-0.5\}$ and $u^{\prime \prime} / u_{\tau} \in\{+0.5,+1.0,+1.5,+2.0,+2.5\}$. The contours in the background give the vorticity field (in $U_{b u l k} / h$ units), and the vectors the transverse velocity fields. In $(a) a=+0.24 U_{b u l k}$ and $b=-0.20 U_{b u l k}$. The number of samples is 997287 for $u^{\prime \prime}>a$ and 1126408 for $u^{\prime \prime}<b$. In $(b)$ the flow fields are also phase averaged $\left(\varphi=60^{\circ}\right.$ in this particular case), with $a=+0.14 U_{b u l k}$ and $b=-0.10 U_{b u l k}$. The number of samples is 18261 for $u^{\prime \prime}>a$ and 18772 for $u^{\prime \prime}<b$. The phase-averaged spanwise velocity is indicated by the thick black line: $(a)$ forcing OFF; $(b)$ forcing ON, $T^{+}=200$. 
Near-wall streak modifications by spanwise oscillatory wall motions
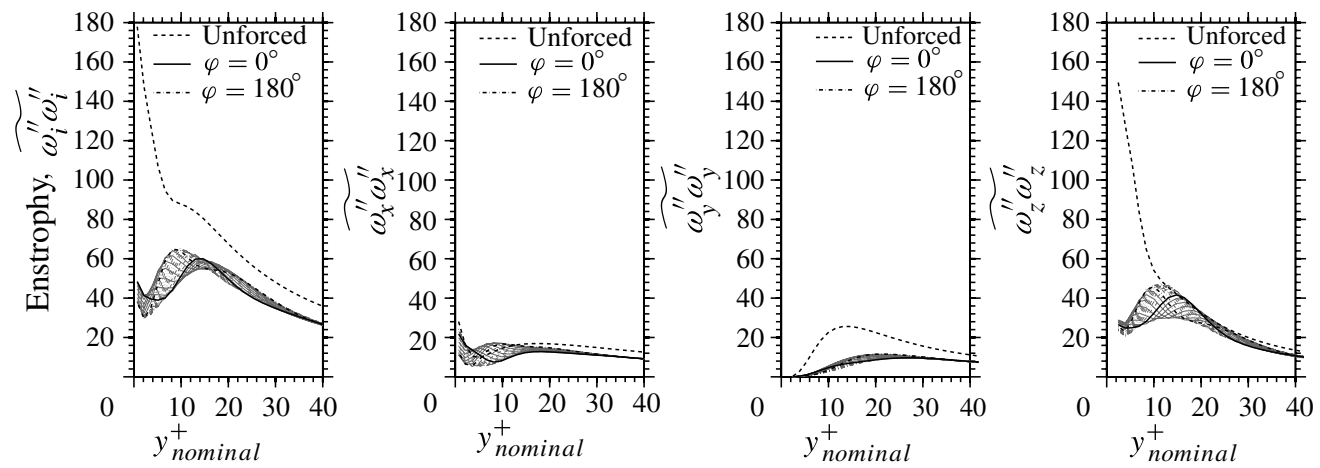

FIGURE 18. Phase-averaged enstrophy and contribution of each component for $\operatorname{Re}_{\tau}=500$ at

$$
T^{+}=100 \text {. }
$$
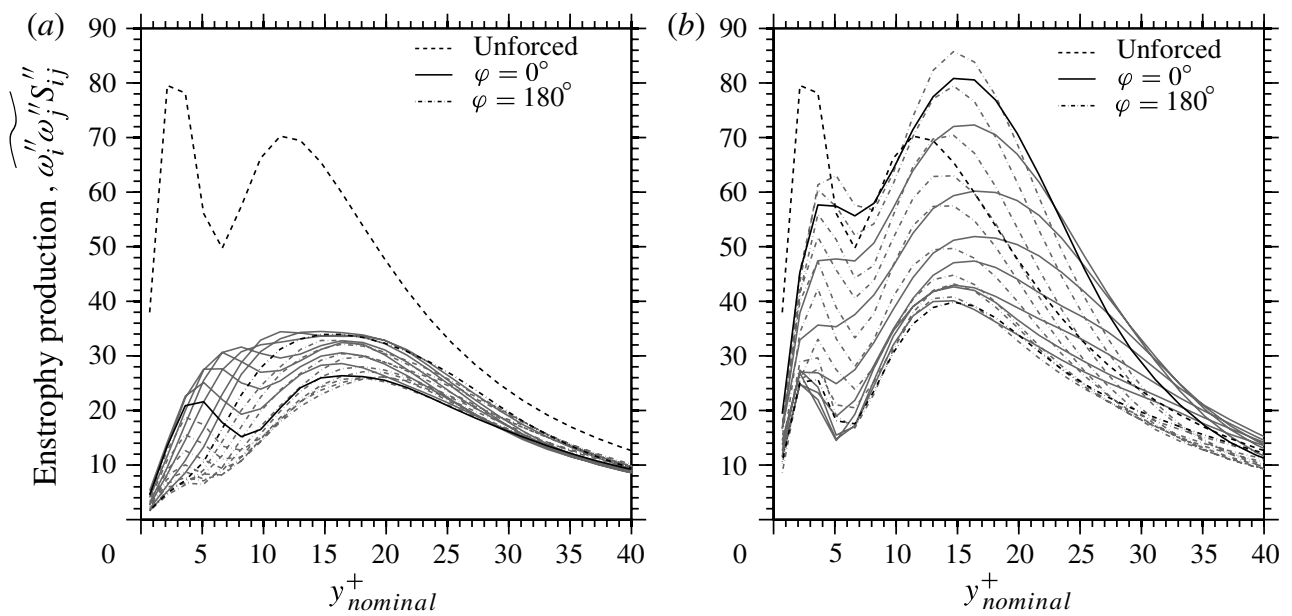

FIGURE 19. Stochastic part of the enstrophy production for $R e_{\tau}=500$ at different phases:

(a) $T^{+}=100 ;(b) T^{+}=200$.

averaged enstrophy and its components for the close-to-optimally forced case relative to the unforced flow, while the latter gives the principal part of the enstrophy production at $T^{+}=100$ and 200. Figure 18 demonstrates, as expected, that the enstrophy reduces significantly in the near-wall region, the phase-related variation being small. Surprisingly, however, this reduction does not arise from a significant decrease in the (already modest) streamwise component in the unforced flow, but mostly from the spanwise component. A reasonable interpretation of this result is that the decrease stems from a strong diminution of the streamwise gradients of wall-normal fluctuations, combined with a flattening in wall-normal gradients of the streamwise fluctuations. Indeed, figure 20 confirms the latter to be the most important contributor, and this is associated with the severe weakening of the streaks. Hence, the conventional close association between streaks and quasi-streamwise vortices appears to be over-rated, insofar as spanwise vortical motions are much more influential. Consistent with the forcing-induced attenuation in total enstrophy is the observation, from figure 19, that the production of enstrophy is strongly reduced. The level of this 

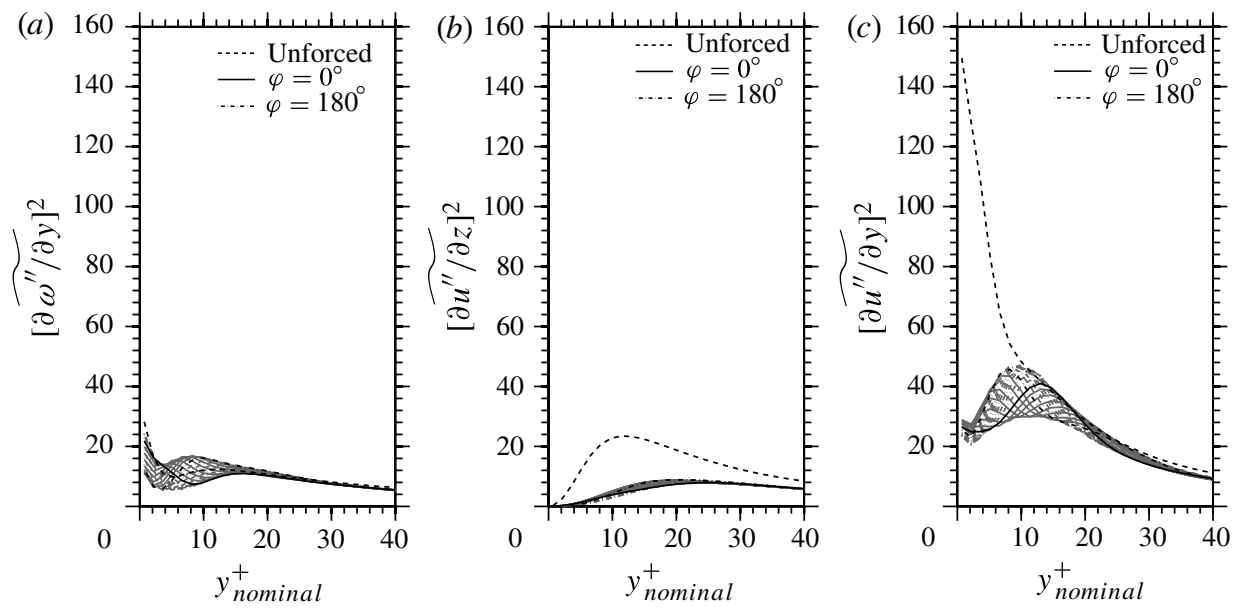

FIgURE 20. Componentwise contributions to the enstrophy for $R e_{\tau}=500$ at $T^{+}=100$ : (a) streamwise; (b) wall normal; (c) spanwise.

decrease is much lower as the forcing period increases to $T^{+}=200$. At the same time, the phase-dependence increases, and this reflects the sensitivity of this process to even minor phase-dependent variations in the near-wall structure, the streak reorientation and phase-variations of the turbulent fluctuations, in particular.

\section{Spectra}

The final set of statistical data presented are the premultiplied power spectral density (PSD) functions shown in figure 21. The spectra, each pair of rows relating to a different stochastic component, are displayed in the form of contours spanning the entire half-height of the channel. In the upper row, for any one component, any spectrum at a given wall-normal distance is normalized by its integral, i.e. the total resolved power at that wall-normal distance. In the lower row, the spectra are normalized by the total power across the entire $f_{\text {nominal }}^{+}-y^{+}$domain. The purpose of the two normalization choices is to identify, on the one hand, any wall-normal variations in the dominant frequency range at which the power density is especially high and, on the other hand, to highlight the most energetic regions, essentially in absolute terms, in both location and frequency. Bright regions indicate high values and dark regions low values. The middle and right-most columns relate to the forced cases, and the respective forcing frequencies are identified by full red dots on the abscissa.

For the unforced flow (left-most column), a first remarkable observation is that the wall-normalized time scale associated with energetic streamwise fluctuations near the wall is of the order of 100 , corresponding to $f^{+}=0.01$. This time scale is closely associated with the transit time of the streaks (relative to a stationary sensor) that can be estimated from the streak length, typically $\lambda_{x}=O\left(10^{3}\right)$, and the representative convective streamwise velocity at $U^{+}=O(10)$ at $y^{+}=10$, at which the streamwisefluctuation energy is also seen to reach a maximum, consistent with the location of the streaks. Whether the coincidence of this time scale with the optimum forcing period is physically significant remains an open question. A second observation is that there is a high degree of uniformity in this time scale across the entire viscous sublayer, a coherence that is only lost well beyond the viscous sublayer. In fact, wall-normal 

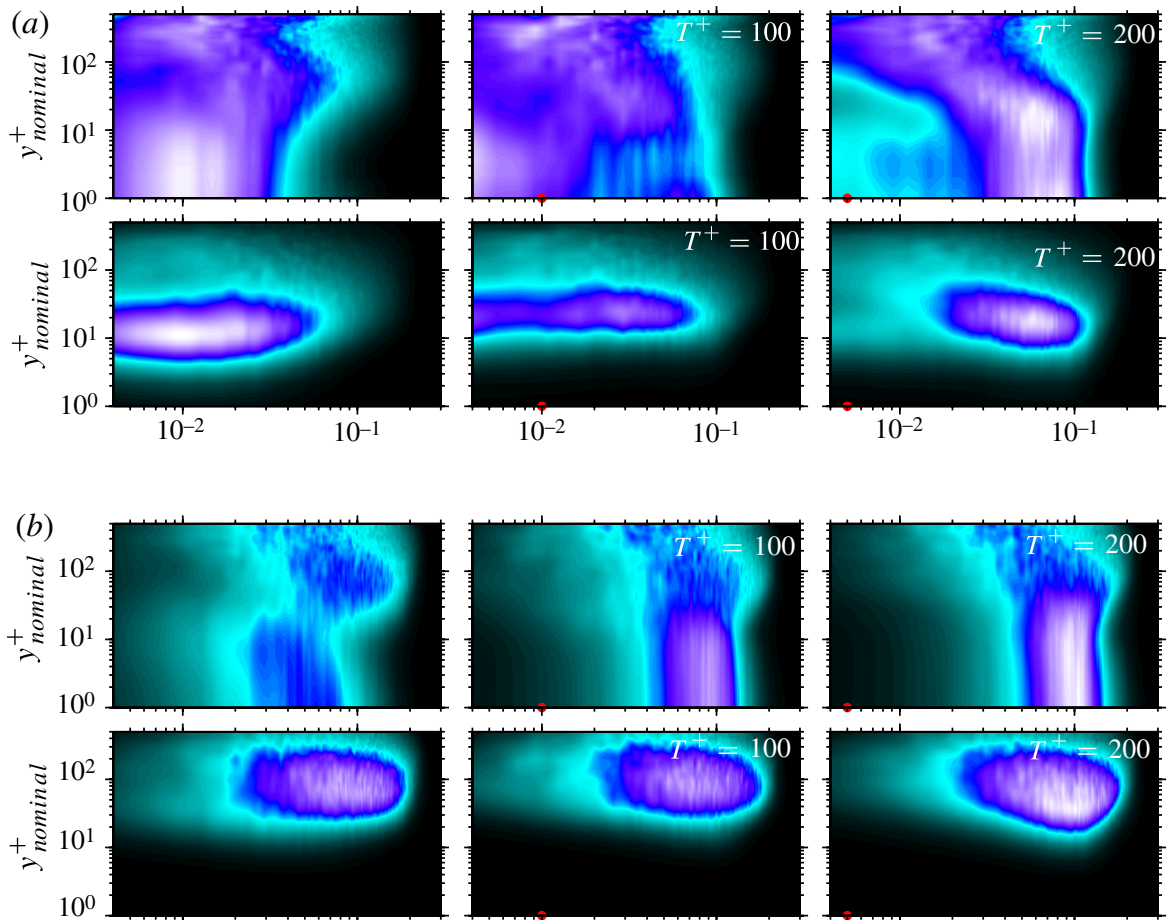

$10^{-2}$
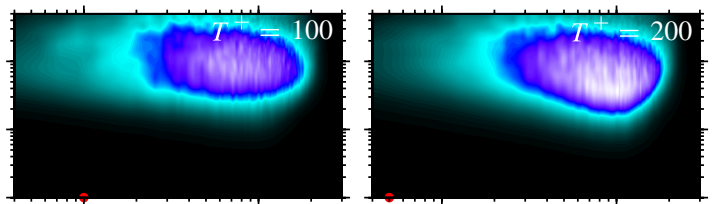

$10^{-1}$

$10^{-2}$

$10^{-1}$

$10^{-2}$

$10^{-1}$
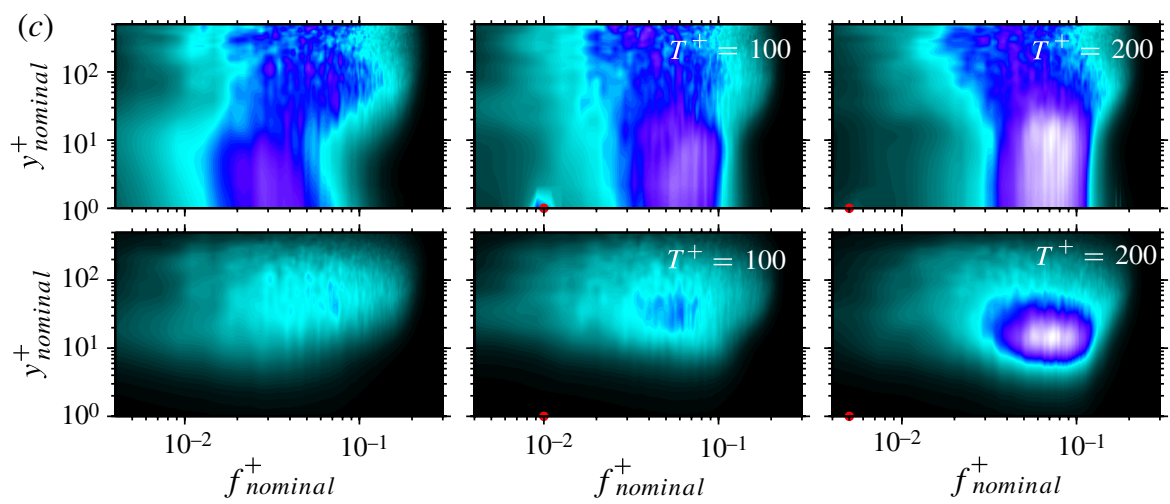

FIgURE 21. (Colour online) Premultiplied power spectral density functions of the velocity fluctuations (at $R e_{\tau}=500$ ). For each velocity component, two rows are given: for the top row, the power spectral density function was multiplied by the frequency and divided by the resolved power at each particular height; for the bottom row, the power spectral density function was multiplied by the frequency and divided by the total resolved power. Each row uses the exact same contour levels, red dots indicate the forcing frequency: $(a) u^{\prime \prime} ;(b) v^{\prime \prime}$; (c) $w^{\prime \prime}$.

coherence of the region of maximum energy is a recurring theme in most of the spectra in the upper rows, including those for the forced flows. The spectra for the wall-normal and spanwise fluctuations are broader and less distinct than that for the streamwise fluctuations, with weak maximum at the considerably higher frequency of 

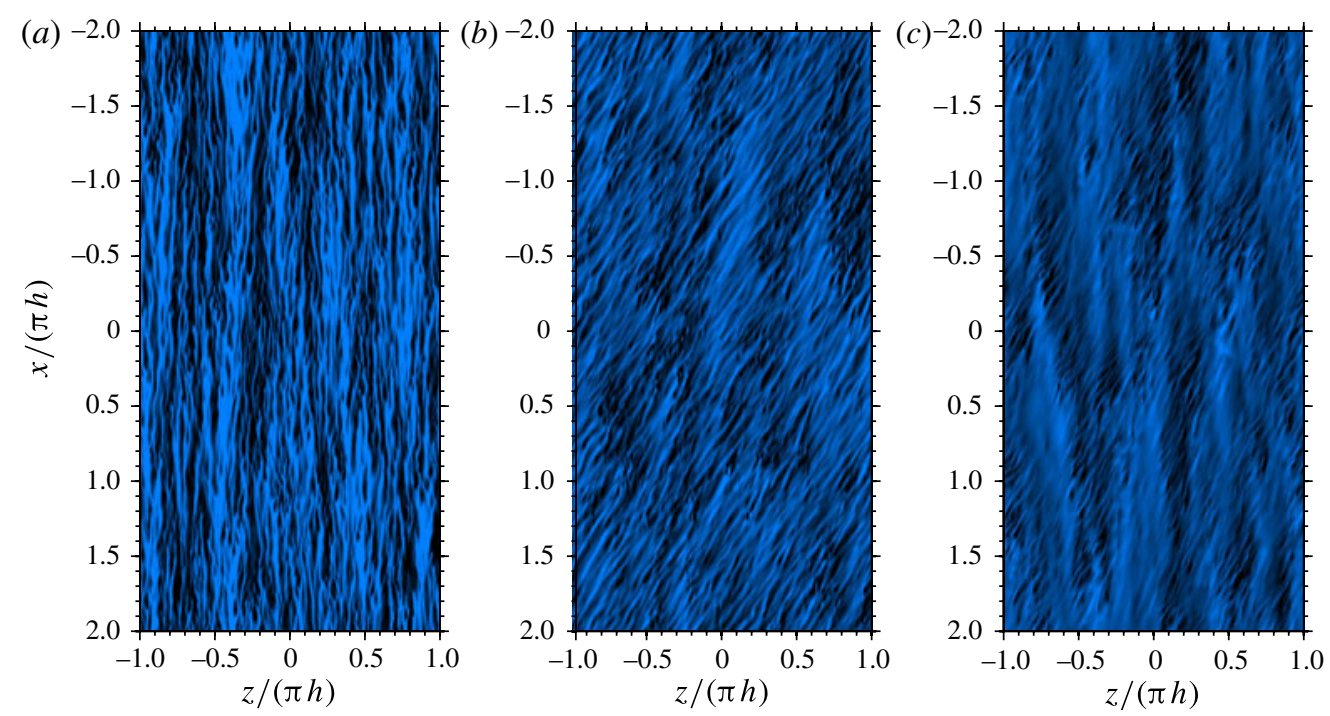

FIGURE 22. (Colour online) Instantaneous snapshots of the streamwise-velocity fluctuations $u^{\prime}$ at $y^{+} \approx 6$ for $R e_{\tau}=500$. The same colour map is applied to $(a),(b)$ and $(c)$ after clipping the data to $u^{\prime} / U_{\text {bulk }} \in[-0.12,0.45]$. Blue (bright) regions correspond to pronounced velocity deficits relative to the mean value. Note the virtual absence of small-scale streaks in $(c)$, where the skin friction drops by $32 \%$ : $(a)$ forcing OFF; $(b)$ forcing ON, $T^{+}=200 ;(c)$ forcing $\mathrm{ON}, T^{+}=100$.

around $f_{\text {nominal }}^{+}=0.04-0.05$ in the viscous sublayer. A reasonable interpretation of this large difference in frequency is that wall-normal sweeps and ejections, and associated spanwise motions, are far more frequent than that implied by the assumed close linkage between streaks and quasi-streamwise vortices. Another possible source is that streaks meander, thus inducing high-frequency fluctuations relative to a 'probe' that is fixed in space, as is the case here. A final observation relating to the unforced flow is that the energy of the wall-normal fluctuations peaks far outside the viscous sublayer, which is consistent with the prevalence of large-scale sweeping motions and the fact that these fluctuations decline at an especially high rate towards the wall, due to the kinematic blockage of the wall.

With forcing imposed, there is a general trend for the maximum energy in all spectra to shift towards frequencies in the range $f_{\text {nominal }}^{+}=0.07-0.1$. This is especially pronounced for the case $T^{+}=200$. It might be regarded as curious, at first sight, that this shift is entirely at odds with the forcing frequency. The explanation is rooted in the fact that the streaks are subjected to periodic tilting (as seen in figure 22). As we discuss later in some detail, a consequence of the forcing, especially at non-optimal forcing frequencies, is a periodic and rapid formation of the streaks at phase values at which the shear-strain angle approaches the two maxima in any one forcing period. At intermediate states, within which the shear angle changes rapidly, the streaks are materially depressed and do not possess a predominant orientation. Relative to a stationary probe, the inclined streaks, separated by distances $\lambda_{z}^{+}=O(100)$, traverse the probe at a speed of $U^{+}=O(10)$, therefore contributing to the fixed-probe spectrum at around $f^{+} \sim 0.1$. The fact that the shift towards this value is only tentative for $T^{+}=100$ is a reflection of the general weakness of the streaks at this near-optimum forcing period. The coherence observed in the viscous sublayer, 
especially at $T^{+}=200$, is again remarkable. It is consistent with the observation that the streak angle does not vary substantially in the wall-normal direction within the viscous sublayer (as we show later), which is counterintuitive in the presence of strong wall-normal variations associated with the Stokes motion. In the turbulent layer, beyond $y^{+}=50$, the frequency range of the energetic streamwise fluctuations remains essentially unaffected by the forcing. This region is characterized by super-streaks, as we show in $\S 11$, which are essentially divorced from the near-wall streaks, although the former will be shown below to modify the latter.

Analogous arguments may be applied to the spectra of the spanwise and wallnormal fluctuations to explain the shift towards $f^{+} \sim 0.1$. An interesting additional feature specific to the $w^{\prime \prime}$ component is the emergence of a pronounced peak at $T^{+}=200$ within the viscous sublayer, relative to the unforced case. This suggests a stronger scale selectivity of the near-wall structures at that particular forcing frequency, as will transpire in the next section, by reference to figure $22(b)$. This may be attributed to the process responsible for the extra production, $\widetilde{\mathscr{P}}_{33}$, shown in figure 15. Equation (B 3c), in appendix B, shows that spanwise fluctuations are enhanced by the interaction between wall-normal fluctuations and the unsteady Stokes strain. This enhancement peaks as the streaks reform and align with the shear angle, the condition which gives rise to the energy maximum in the near-wall spectra being around $f^{+} \sim 0.08$. The fact that the absolute energy maximum has drifted down from around $y^{+}=50$ towards $y^{+}=10-15$ strengthens the above interpretation, because this drift is consonant with the maximum in $\widetilde{\mathscr{P}}_{33}$ also occurring at this latter wall-normal distance.

\section{Flow structures}

\subsection{Streak inclination and strength}

The characteristics of streaks in canonical near-wall shear are well-researched, and are not the subject of the following discussion. Rather, interest is focused herein on the response of the streaks to the oscillatory wall forcing. The fact that the forcing causes the elongated near-wall structures to tilt is known. Both Choi (2002) and Ricco (2004) present hydrogen-bubble images that show inclined traces, which may (albeit rather loosely) be interpreted as 'streaks'. The terminology requires care, because streaks are defined as regions of elevated or depressed streamwise velocity, and this is not what the visualizations show. Inclined streaks are also evident when spanwise motion is induced by an oscillating Lorentz force, as shown by Du et al. (2002). The reorientation of the streaks, as part of their general response to forcing, is not merely an inert kinematic manifestation of the response, but is an important indicator of the near-wall dynamics and thus the drag-reduction process. Choi (2002) provides an interpretation of how streaks are made to incline by the transverse motion, and this has been mentioned already in $\S 1$. The explanation rests on the assumption that the streak inclination is driven directly by the spanwise wall velocity. It is shown below that the inclination is governed, rather, by the direction of the shear-strain vector, which is entirely different from (indeed often opposed to) the direction of wall velocity. Furthermore, Choi's paradigm includes the assumption that the vorticity sheet associated with the shear in the Stokes layer is periodically tilting, thereby generating mean spanwise vorticity, but it is entirely unclear how this can be effected in channel flow.

Figure 22 shows three snapshot of streamwise-velocity fluctuations at $y^{+}=6$, the middle and right-hand side images displaying typical streak-inclination patterns 


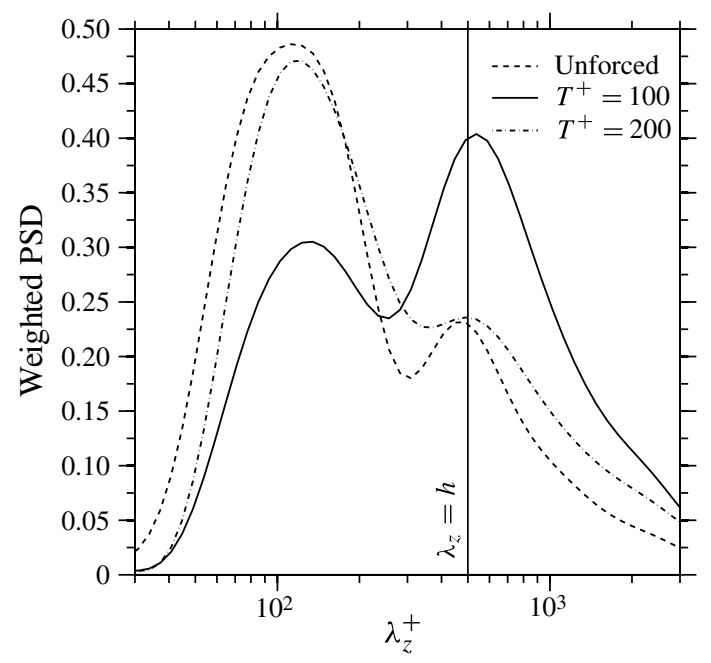

FIGURE 23. Spanwise spectrum of the streamwise-velocity fluctuations at $R e_{\tau}=500$ for $y^{+} \approx 6$. The spectrum was acquired over 2162 viscous time scales with a sample taken every 0.72 viscous time units, where each sample comprises the entire channel length, totalling about $2.3 \times 10^{6}$ realizations. All spectra are then made smoother by applying a sixth-order non-causal Butterworth filter.

observed during the forcing at $T^{+}=200$ and 100, respectively (the visualization window width is $\sim 3000$ wall units). A purely visual inspection allows four statements to be made: first, in the absence of forcing, there are $\sim 30$ streaks within the spanwise domain, and this suggests, much as expected, a streak distance of the order $10^{2}$ of wall units; second, there is clear evidence of larger structures, 'super-streak', in both the unforced flow and the forced flows, which interrupt the regularity of the near-wall streaks, with roughly five such structures visible across the spanwise box; third, there is clear evidence of the forcing weakening the streaks; fourth, at the near-optimum actuation period, there exist extensive regions which are effectively devoid of the streaky structure, and it appears that the distribution of these regions is dictated by the large-scale structures, i.e. the intensity of the small-scale structures is influenced by the large-scale structures. We show later that this interaction also gives rise to a non-negligible impact on the wall-shear stress.

Figure 23 provides statistical evidence, by way of distributions for the weighted PSD, for the inter-streak distances noted above. All three curves feature two maxima, at $\lambda_{z}^{+} \approx 120$ and 600 , the former identifying the near-wall streaks and the latter the large outer streaks which are separated by about one channel half-height. At the near-optimum forcing period, $T^{+}=100$, the near-wall streaks are strongly damped, and the footprint of the outer streaks becomes dominant. At $T^{+}=200$, the near-wall streaks are weaker, but evidently not as weak as might be anticipated in the light of the statistical results discussed earlier. Reference to the images in figure 22 suggests that the streaks at this longer period are much more pronounced and well formed than at the optimum value. There is an indication of a slight rise in streak spacing, around $10 \%$ at $T^{+}=100$, and this is broadly in line with observations by Ricco (2004).

The correspondence between the shear angle and the orientation of the streaks is explored in figure 24 with the aid of 12 snapshots of the streak structure at $T^{+}=200$, each row pertaining to one particular phase and giving views at three wall-normal 

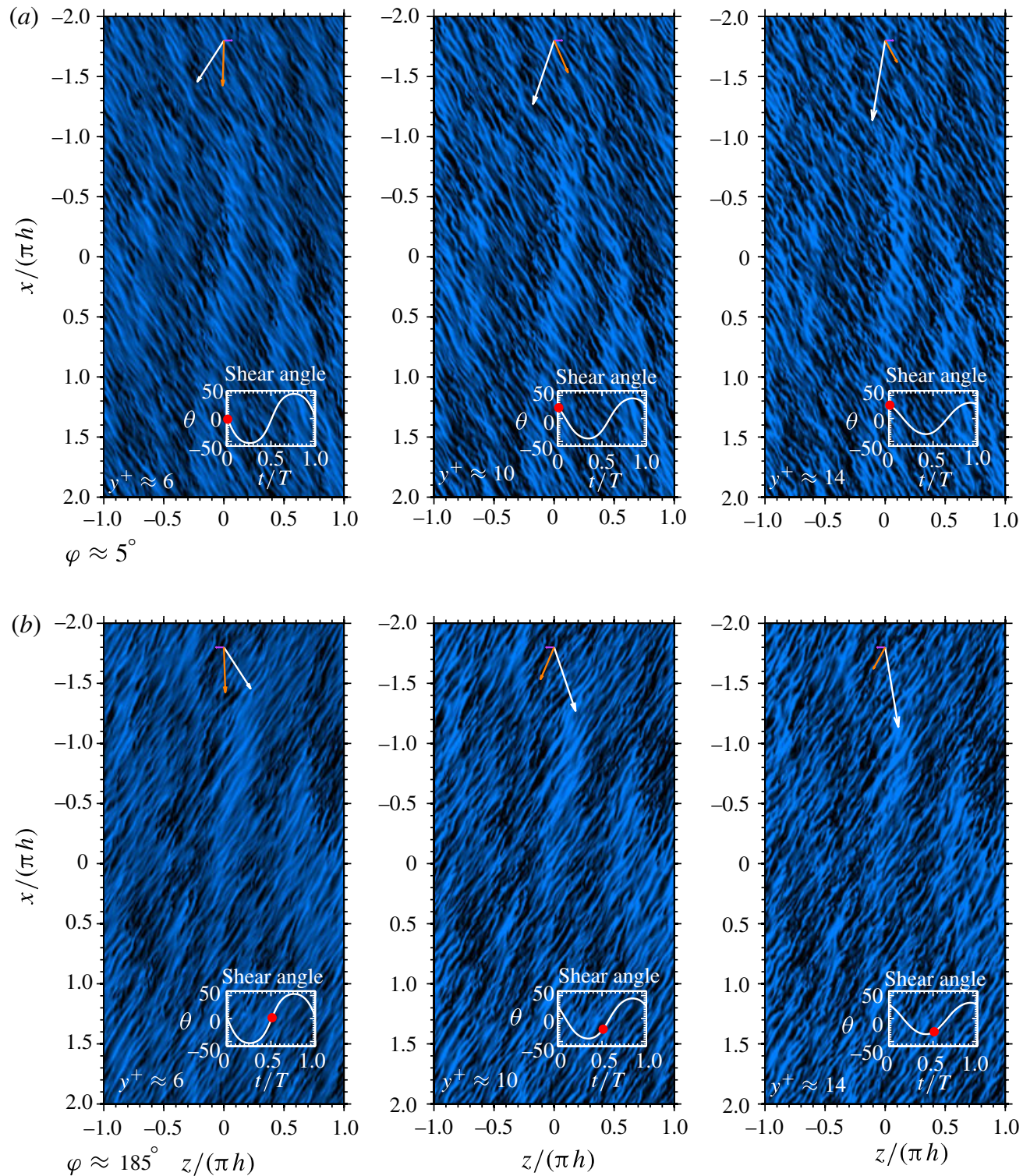

FIGURE 24. For the caption, see the next page.

distances. The choice of $T^{+}=200$, in preference to 100 , is rooted in the observation that the streak structure at the former value is more distinct at all phase angles.

The vectors given at the top of plots relate to the spanwise wall velocity, the local phase-averaged velocity and shear-strain rate (see caption). The angle of the last, the shear angle, is indicated by a full circular symbol in the inset that shows the temporal variation of this angle over the forcing period, $t / T=0$ and 0.5 corresponding to the zero-velocity states of the wall.

There are four main observations that derive from the visualizations. First, the direction of the streaks is only a weak function of the wall-normal position within a major proportion of the viscous layer, the variations being rather weak compared with the larger wall-normal change in the direction of the phase-averaged flow and 

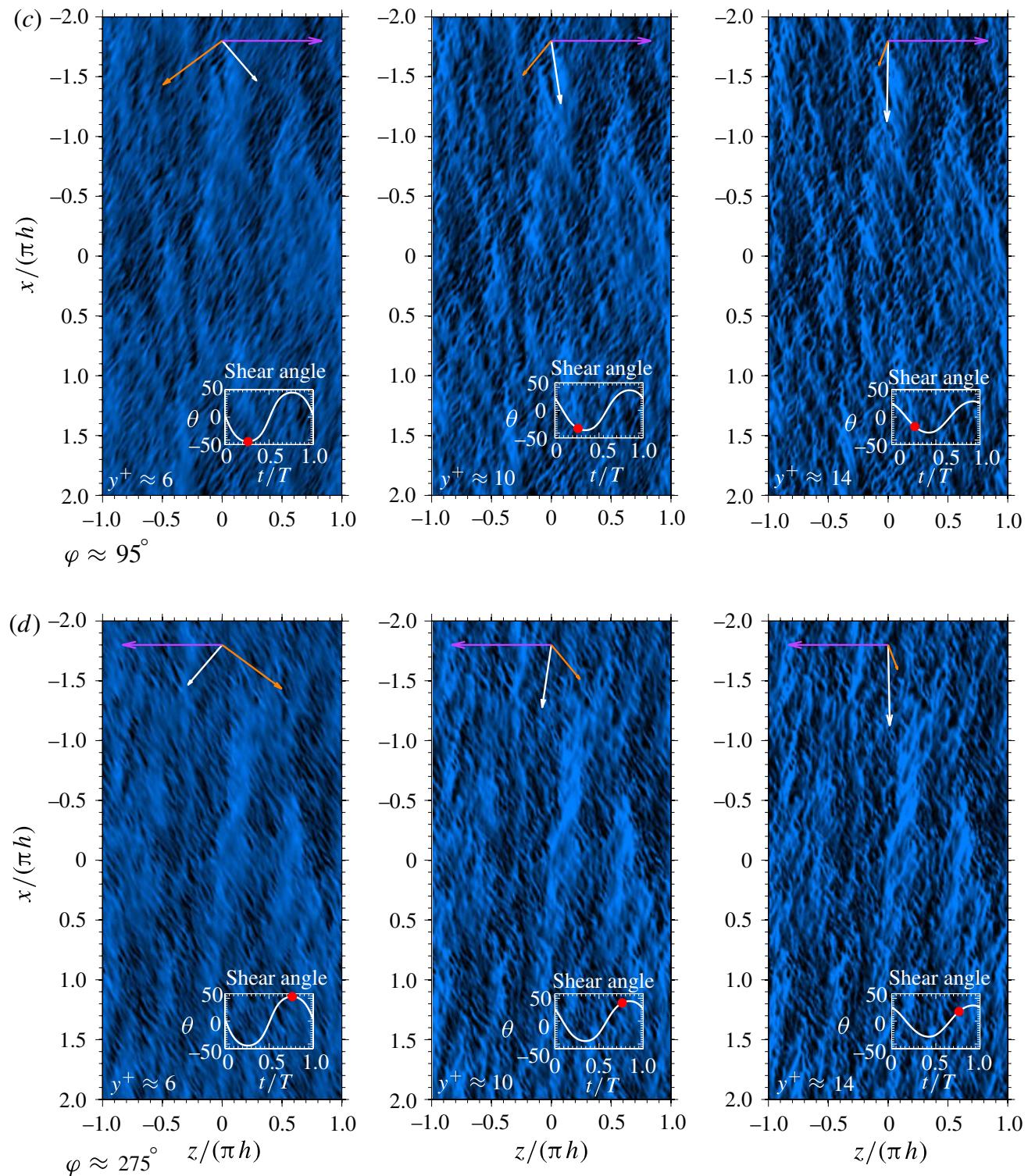

FIGURE 24. (Continued) Instantaneous snapshots of the streamwise velocity fluctuations $u^{\prime}$ at $y^{+} \approx[6,10,14]$ (left to right) for $R e_{\tau}=500, T^{+}=200$. The same colour map is applied everywhere after clipping the data to $u^{\prime} / U_{\text {bulk }} \in[-0.12,0.45]$. Blue (bright) regions correspond to pronounced velocity deficits relative to the mean value. The white arrow corresponds to the vector $[\tilde{w}, \bar{u}+\tilde{u}]$, the orange arrow to $[\partial \tilde{w} / \partial y, \partial[\bar{u}+\tilde{u}] / \partial y]$ while the purple arrow corresponds to the wall velocity. Animated versions of this figure, together with the baseline and $T^{+}=100$ cases, are available from the authors upon request.

the shear vector. Aspects of wall-normal coherence have already been highlighted in earlier parts of the discussion, notably in relation to the spectra maps. Second, there is a pronounced trend towards an alignment of the streaks with the shear-strain vector around the location $y^{+}=10$ and above, within which the bulk of the streak dynamics is likely to be most vigorous. Third, when the spanwise strain changes at a 

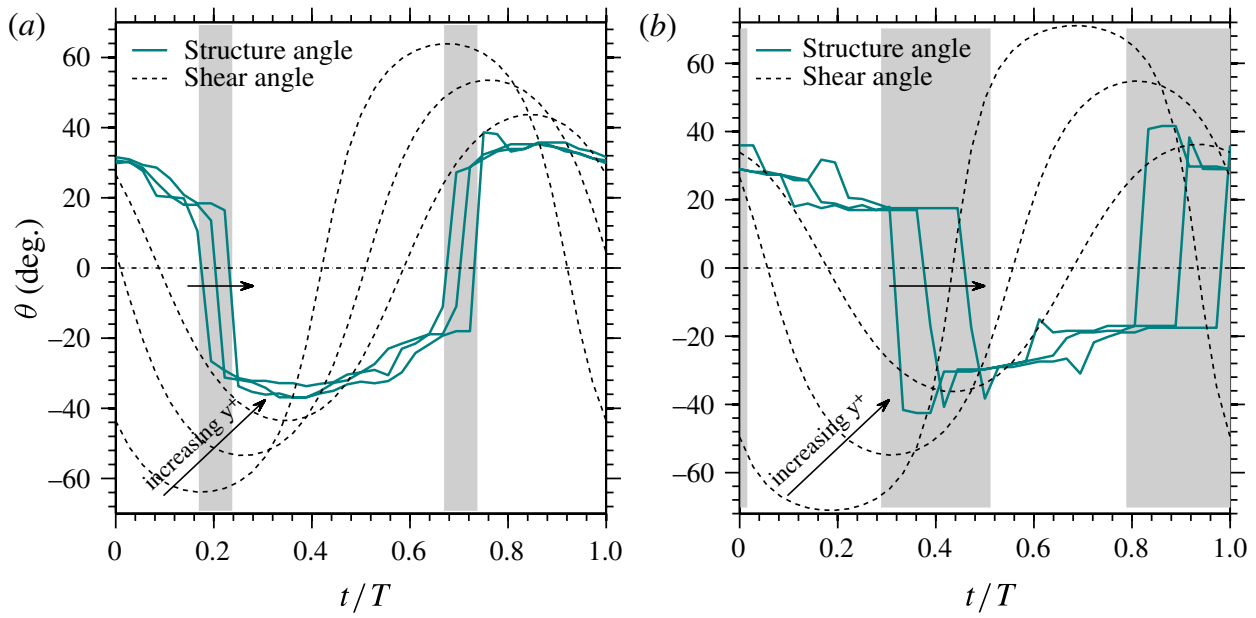

FIGURE 25. (Colour online) Streak and shear angles as a function of phase for $y^{+} \in\{2,6,10\}\left(\operatorname{Re}_{\tau}=500\right):(a) T^{+}=200 ;(b) T^{+}=100$.

high rate, with some time delay following the wall-velocity reversal, the shear-vector angle changes rapidly, in terms of both direction and magnitude, and the streaks are observed to weaken substantially, losing their distinct orientation. Fourth, the streaks tend to be most vigorous, or clearly established, as well as most strongly tilted, in two conditions, namely when straining due to the Stokes motion is shortly beyond its extreme values in the layer $y^{+}=O(10)$. In these states the shear vector tends to change little in time, in terms of both direction and magnitude, over a proportion of the forcing period, a state referred to herein as 'lingering', and this encourages the (re-)generation of the streaks in the direction of the 'lingering' shear strain. The implication of this behaviour is that the ratio of forcing time scale to that governing the formation of streaks in response to (quasi-steady) shear is of major significance.

The correspondence between the shear angle and the orientation is quantified in figure $25(a)$ for $T^{+}=200$, at $y^{+}=2,6$ and 10 . The streak angle was determined from streak distances in the $x$ - and $z$-directions extracted from related phase-averaged two-point correlation functions. These were constructed from the high-pass-filtered velocity field in Fourier space (with sharp cut-off corresponding to 200 and 1500 wall units, in the structure-normal and structure-parallel directions, respectively), so as to remove large-scale contributions. The sign of the angle, which cannot be determined from the above, is derived visually from images such as those in figure 24.

It is observed that the variation of streak angle follows that of the shear angle at $y^{+} \sim 10$, but has a close-to-bimodal character; that is, there exists a well-defined streak orientation over a proportion of the shear-angle period in which the shear angle 'lingers', and no distinct structure is observed as the shear angle varies rapidly. The implication is thus that the streaks recover rapidly once the rate-of-change of the shear angle falls below some threshold and for a sufficient period of time. In addition, figure 25(a) illustrates the close correspondence of the streak angle at all three wall-normal planes with the shear angle in the central portion of the viscous sublayer, and also the wall-normal near-constancy (coherence) of the angle.

An analogous plot for $T^{+}=100$ is shown in figure 25(b). For this forcing period, the streaks are weak, and the determination of their angle difficult and subject to significant uncertainty. Hence, the results should be regarded merely as providing 
qualitative trends. In common with $T^{+}=200$, the streak-angle variation tends to be bimodal. Wall-normal uniformity of the angle is somewhat less pronounced, but still clearly discernible. The more pronounced wall-normal differences in the streak-angle variations at $T^{+}=100$ is consistent with the larger wall-normal phase shift in the shear angle at this forcing period (see figure $25 \mathrm{~b}$ ). The extrema in the shear angle occur at $t_{1} / T=1 / 8+y_{p}^{+} / \sqrt{4 \pi T^{+}}$and $t_{2} / T=5 / 8+y_{p}^{+} / \sqrt{4 \pi T^{+}}$for a given wall-normal distance $y_{p}$ (based on Stokes solution), yielding a $1 / \sqrt{T^{+}}$dependence in the phase shift normal to the wall.

The phase variation of the streak properties and orientation angle is of interest to those who attempt to predict the turbulence near-wall structures by linear analyses of the Navier-Stokes equations. Such analyses aim to identify the formation of streaks, signified by the amplification of perturbations and various measures of the streamwisefluctuation energy given a prescribed mean-strain field. The amplification process extends over a time $\tau_{a}$, which is associated with the streak-formation time scale. In the case of the unforced channel, this time scale is $\tau_{a}^{+} \sim 50$ (see Blesbois \& Chernyshenko 2011). The present investigations suggest that, following a scrambling phase in which the streaks are substantially weakened, there is a phase (period) of near-constant shear strain, $\tau_{s}$, during which the streaks amplify and re-organize. The implication is, thus, that this process is closely connected to $T^{+}$. In particular, it appears that $\tau_{s} / T \sim 1 / 4$ (see figure 25). To allow for the streaks to form, the condition $\tau_{s}>\tau_{a}$ thus translates to $T^{+}>4 \tau_{a}^{+} \approx 200$, which is consistent with the DNS observations where near-wall streaks remain suppressed for $T^{+}=100$ but start re-organizing when $T^{+}=200$.

The above discussion clearly established that the near-wall turbulence structures change significantly from one phase to another. However, the link with the time- or phase-averaged skin friction is not straightforward as the skin friction reflects the wall-integrated process rather than the footprint of individual structures. For example, the phase-averaged skin friction in the streamwise direction depends on both $\mathrm{d} \bar{u} / \mathrm{d} y$ and $\partial \tilde{u} / \partial y$ at the wall. As noted during the discussion of (4.2), the contribution of $\tilde{u}$ is negligible for the $T^{+}$values of interest, and thus the phase variation of the skin friction is also negligible (less than $1 \%$ of the time-averaged value). This may seem contradictory, at first sight, given the phase-dependence of the turbulence structures, but the link with the skin friction is through the Reynolds shear stress, which is a very weak function of the phase (as already discussed when examining (4.2a)) and therefore leading to an almost phase-independent skin-friction value. This was also established when discussing the very weak phase dependence of the phase-averaged probability distributions of $u^{\prime \prime}-v^{\prime \prime}$ in figure 9 . The role of the modified turbulence structures is to cumulatively reduce the correlation between $u^{\prime \prime}$ and $v^{\prime \prime}$, and the weak phase dependency simply reflects the fact that in the close-to-optimal situation, the near-wall structures are not allowed a sufficient amount of time to organize themselves, in which case the shear-stress modulation in $(4.2 a)$ is suppressed.

\subsection{Transverse streak structure}

Snapshots of the transverse motion, in concurrence with contours of streamwisevelocity fluctuations, do not provide much insight into the effect of the forcing, except insofar as they illustrate a general weakening in turbulence activity, especially close to the wall. This is illustrated by the two snapshots in figure 16, which contain vectors of the cross-sectional velocity-fluctuation field, with the phase-averaged fluctuations removed. Apart from illustrating the substantial weakening in cross-flow motions resulting from the forcing, it verifies, qualitatively, that the streak distance in the 
unforced case is indeed of order $10^{2}$ wall units, and it indicates an attenuation in the intensity of the streaks, especially very close to the wall.

Much more informative are conditionally sampled fields. This technique has been used by Choi et al. (2002) to examine the forcing-induced deformations of streaks in oscillatory pipe flow at $R e_{\tau}=170$, subject to sampling being conditional on streamwise vortices. In contrast, the present approach targets the streaks themselves, with sampling being conditional on the detection of maximum/minimum stochastic streamwise fluctuations at $y^{+} \approx 10$.

To provide a background against which the effects of forcing can be discussed, figure 17 gives a ensemble-averaged fields, conditional on low- and high-speed streaks, for the unforced and forced flows. The line-contours identify isostreamwise-velocity defect or excess, relative to the time-averaged field (note that the isocontour values are normalized by $u_{\tau}$ ), and the vectors represent the (conditionally sampled) cross-flow velocity field. In line with expectations, the unforced fields show a close relationship between low-speed regions and ejections (anti-splats), on the one hand, and highspeed regions and sweeps (splats), on the other. The plots also bring out the quasistreamwise vortices associated with the ejections and sweeps. Because the lateral extent of either image is 120 wall units, the two images, if put side by side, roughly cover the statistical inter-streak distance of around 120 wall units.

With forcing present, the streak structure and the cross-sectional flow field are much more complicated. This emerges from figure 26, which shows phase-averaged visualizations of the high- and low-speed streaks in the left-hand and right-hand side columns, respectively, at six phase angles of the forcing at $T^{+}=200$. Again, this non-optimum forcing period has been chosen deliberately, allowing the response of the streaks to be identified with greater clarity. Each plot contains three sets of data, all being conditionally sampled, by reference to the streak 'centres' at $y^{+}=10$, and phase averaged: line contours of the streamwise velocity; vectors of cross-flow stochastic velocity fluctuations $\left(v^{\prime \prime}, w^{\prime \prime}\right)$; colour contours of the ratio between the conditionallysampled, phase-averaged value of $f$, denoted by $\langle f\rangle_{p h .+c o n d .}$, and its time-averaged value $\bar{f}$, with $f$ being $v^{\prime \prime} \partial \tilde{w} / \partial y$ and $v^{\prime \prime} v^{\prime \prime}$ in consecutive sets of plots. In addition, profiles of the Stokes velocity and its wall-normal gradient are included, the latter being identified by open circular symbols. The rationale underlying the inclusion of the stresses in the form shown is to identify specific regions and phases in which the Stokes motion tends to preferentially suppress the stresses below their time-averaged values, thus promoting drag reduction. In this context, it is appropriate to emphasize that the present results have been obtained at a flow condition which represents the cumulative effect of the forcing; that is, the visualizations relate to a flow in which, statistically, the drag reduction is essentially low and stable. Hence, any phase-related events can only be used to identify trends in particular regions, indicating a positive or negative contribution towards a further depression of turbulence activity relative to the mean. In the discussion to follow, reference is made to (B $3 a$ ) and (B $3 c$ ), which describe the stochastic fluctuations. Attention is drawn, in particular, to the generative terms (i) and (ii), which link the stochastic fluctuations to the mean streamwise strain and phase-averaged Stokes strain.

One response to the Stokes straining that emerges most strikingly from the plots is the strong convective distortions of the streaks in the viscous near-wall layer. In particular, near-wall portions of the low-speed streak are being pulled towards and below the neighbouring high-velocity streak, and superficially analogous distortions arise in respect of the high-speed streaks. The maximum of this distortion is 

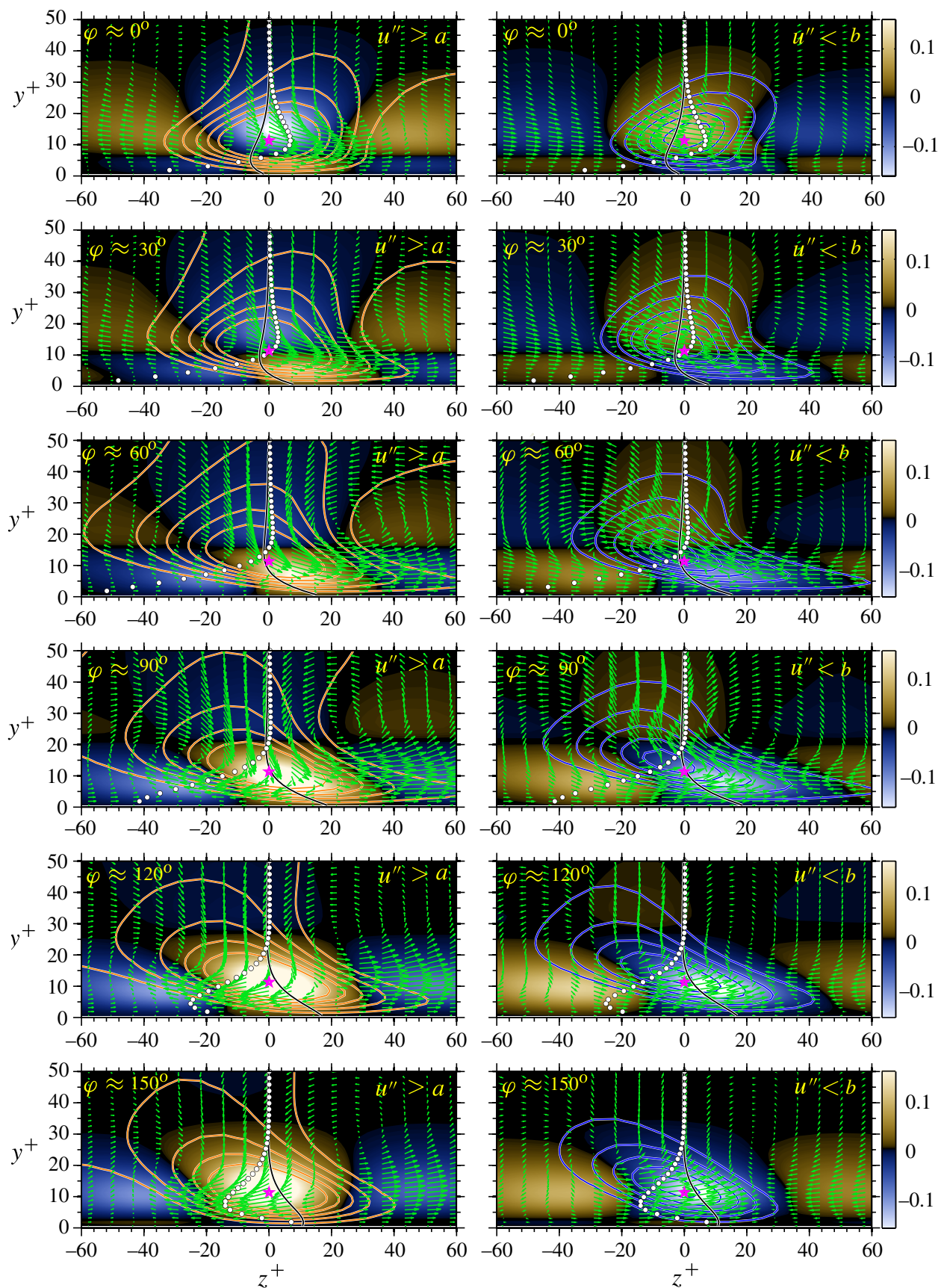

FIGURE 26. Phase-averaged and conditionally averaged streaks obtained by sampling the flow, after application of a high-pass filter to remove super-streak modulations, such that $u^{\prime \prime}<b$ and $u^{\prime \prime}>a$ at $y^{+} \approx 10$ (magenta star). Contour lines give the streamwise velocity contours $u^{\prime \prime} / u_{\tau} \in\{-2.5,-2,-1.5,-1,-0.5\}$ and $u^{\prime \prime} / u_{\tau} \in\{+0.5,+1.0,+1.5,+2.0,+2.5\}$. The contours in the background give $\left\langle v^{\prime \prime} \partial \tilde{w} / \partial y\right\rangle_{p h .+ \text { cond. }}$. The vectors give the transverse velocity fields. In all cases $a=+0.14 U_{\text {bulk }}$ and $b=-0.10 U_{\text {bulk }}$. The phase-averaged spanwise velocity is indicated by the thick black line (which is scaled on the velocity field) and its wall-normal derivative is indicated by the white dots (not to scale). 

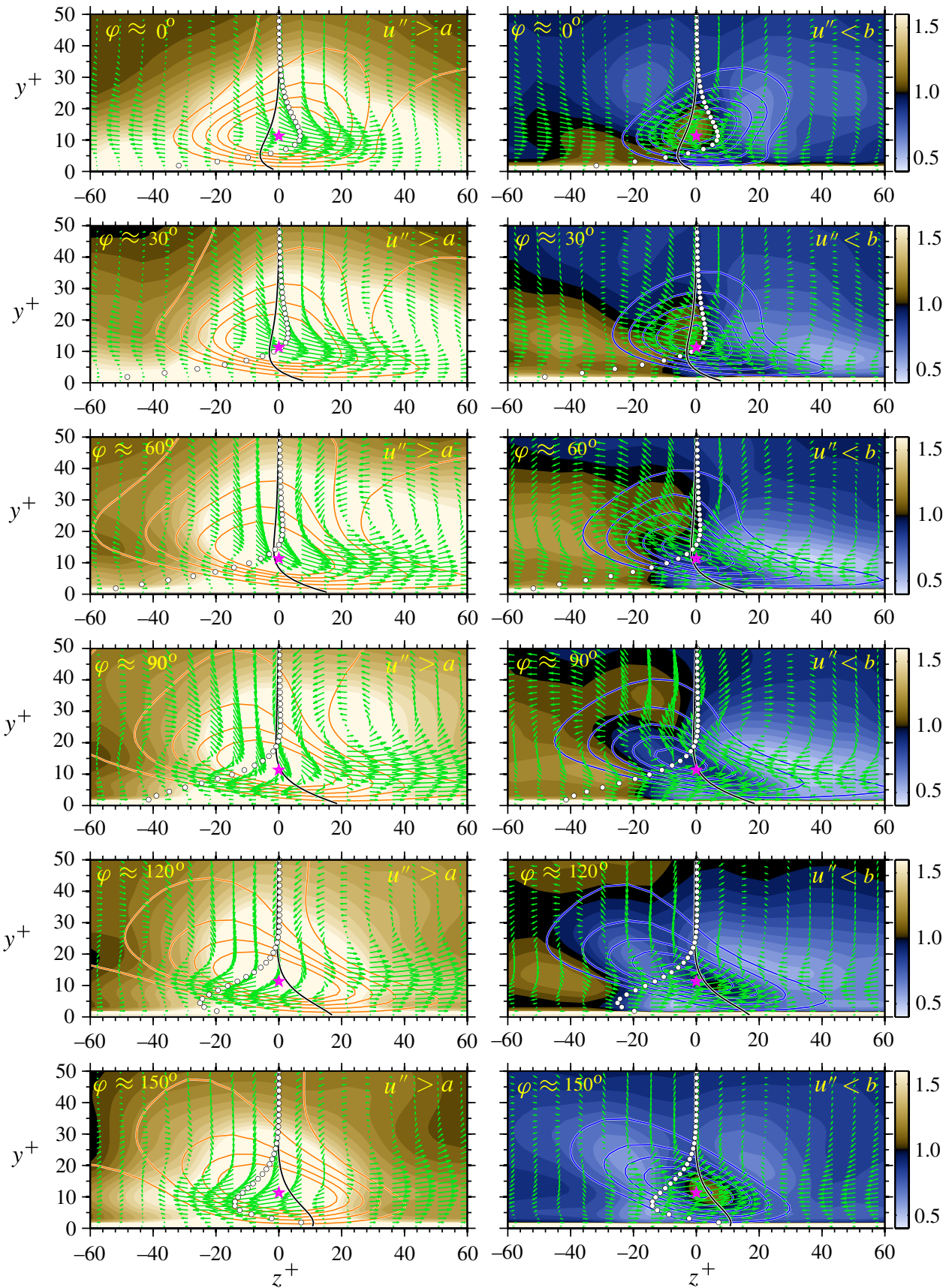

FIGURE 26. (Continued)—but with the background contours giving $\left\langle v^{\prime \prime} v^{\prime \prime}\right\rangle_{\text {ph.+cond. }} / \overline{v^{\prime \prime} v^{\prime \prime}}$.

coincident with the peak wall velocity $\left(\varphi=90^{\circ}\right.$ in figure 26), and is therefore mainly driven by direct convection associated with $\tilde{w}$ in the Stokes layer (note that a similar process occurs at $\varphi=270^{\circ}$ ). Further away from the wall, the generation term (ii) in (B $3 c$ ) becomes more significant and reaches its extrema at $\varphi \approx 120^{\circ}$ and $\varphi \approx 300^{\circ}$ for $y^{+}=10$ and $T^{+}=200$. At these conditions, the streaks undergo 
maximum amplification and assume their characteristically oblique orientations (see also figure $25 a$ ).

Below the low-speed streaks, the wall-normal fluctuations are predominantly positive, so that the action of term (ii) in (B 3c) is to generate spanwise fluctuations that are mostly in line with the Stokes motion, thus enhancing spanwise convection for all three fluctuating components (this is based on the observation that at any given height $y^{+}, \tilde{w}$ and $\partial \tilde{w} / \partial y$ are of opposite signs for $\sim 79 \%$ of the time, using the Stokes solution). This additive process emerges clearly from the right-hand side plots in figure 26. Conversely, the wall-normal velocity fluctuations around the high-speed streaks are predominantly negative, causing the spanwise fluctuations to be opposed to the Stokes motion, thus weakening the convective distortions of the streaks (although the maximum strength of each action occurs at different heights, as noted earlier). Again, this is clearly displayed by the plots on the left-hand side of figure 26 . There is, therefore, an inherent asymmetry in the spanwise distortions of the low- and high-speed streaks, with the former affected more strongly, especially around $y^{+}=10$, where the generation of the spanwise fluctuations reaches a maximum.

The preferential distortion of the low-speed streaks described above leads to one plausible facet, i.e. possibly part of the story, of the drag-reduction paradigm. This is based on the deduction that the Stokes motion gives rise to a preferential inundation of the near-wall region by low-speed fluid, driven by the mutually reinforcing phaseaveraged and fluctuating spanwise component, a process that depresses the near-wall streamwise shear strain, as near-wall low-speed streaks induce a weaker wall-normal velocity gradient.

A second component of the drag-reduction mechanism is proposed to be linked to the strong distortions of the cross-flow fluctuations by the Stokes motion. A consequence of the alternate generation of negative and positive spanwise fluctuations under the low-velocity and high-velocity streaks, respectively, is the formation of highly skewed ejections and sweeps, with motions almost parallel to the wall. Both sweeps and ejections are especially evident on the upper right-hand sides of the stretched streak-velocity contours in figure 26. In both low-speed and high-speed streaks, the ejection or sweep motions are deflected upwards by the opposing spanwise motions generated below the streaks. It is reasonable to postulate that the strongly spanwise-oriented motions close to the wall 'shield' the viscous sublayer from wallnormal splatting and, as a consequence, anti-splatting. This suppression is, furthermore, presumed to be reflected, statistically, by the decline in pressure-velocity interaction and the r.m.s. value of the wall-normal pressure-fluctuation gradient (figures 11 and 13).

Another important aspect of the production term (ii) in (B 3c) is the role it can play in disrupting a previously established unforced flow. Starting from a baseline flow, where streaks align with the vector formed by $[\mathrm{d} \bar{u} / \mathrm{d} y, 0]$ (in a plane parallel to the wall), and upon a sudden imposition of a constant spanwise-oriented shear $\partial \tilde{w} / \partial y$, it is clear from the above discussion that a new set of streaks form and align with $[\mathrm{d} \bar{u} / \mathrm{d} y, \partial \tilde{w} / \partial y]$. In this process, the first set of streaks (from the baseline flow) is essentially 'destroyed' by the new set provided that $\partial \tilde{w} / \partial y$ is sufficiently large, relative to $\mathrm{d} \bar{u} / \mathrm{d} y$. Naturally, if $\partial \tilde{w} / \partial y$ is now allowed to change in time, the most recent set of streaks is continuously disrupted to form a newer set, and if the rate of change of $\partial \tilde{w} / \partial y$ is carefully chosen, it is possible to reach a point where near-wall streaks are never observed because they are not allowed a sufficient amount of time to form. This is, presumably, the optimal scenario to achieve the lowestpossible turbulence skin friction. In the present scenario (the Stokes layer), and for 
the conditions under investigation, $\partial \tilde{w} / \partial y$ is up to three times larger than $\mathrm{d} \bar{u} / \mathrm{d} y$ at the wall, rendering $\partial \tilde{w} / \partial y$ the dominant component in the formation process. In fact, the higher magnitude of $\partial \tilde{w} / \partial y$, relative to $\mathrm{d} \bar{u} / \mathrm{d} y$, goes some way towards explaining the visual observation that the streaks formed at $T^{+}=200$ appear to be more organized than for the baseline flow (see figures 22 and 24 for example and the corresponding animations, available from the authors upon request), confirmed by the more selective nature of the spectrum in figure 21. The linear analysis of Blesbois \& Chernyshenko (2011) also suggests higher growth rates with forcing, compared to the baseline case.

Some final observations are made about the colour contours in figure 26, which may be argued to point to the processes which are primarily responsible for driving the unforced state towards the final reduced-drag forced state. It is recalled first that the contours identify departures of the local $v^{\prime \prime}-v^{\prime \prime}$ correlations from their respective time- and spanwise-averaged values. With attention focused on the wall-normal stress, it is observed that this is strongly depressed in the region into which the Stokes motion drags the low-velocity streak. This region is characterized by a near-horizontal (spanwise) motion and weak wall-normal motion. While this depression must be counterbalanced by an excess around the high-speed streak, the essential point is that forcing appears to drive the flow towards a lower-drag state primarily by suppressing (or depressing) ejections associated with the low-speed streaks.

\section{Interactions with outer-layer turbulence structures}

The present section addresses the relationship between outer and inner streaks. Although it is well established (e.g. Marusic et al. 2010) that large-scale outer motions contribute to the near-wall turbulent state, both by way of 'foot-printing' and through a 'modulation' of the small-scale near-wall streaks, this subject has received very little attention in the context of friction-drag reduction by oscillatory wall motion. A recent DNS study addressing the relative contribution of large- and small-scale structures on the skin friction is that of Fukagata, Kobayashi \& Kasagi (2010). This study addressed, principally, the consequences of damping the outer structures, by an application of an artificial body force, to the skin friction, but also included a limited statistical analysis identifying the contribution of different scales to the second moments and shear-stress budget. However, it did not deal with the structural details of the interaction.

In the discussion of figure 22, the simultaneous presence of both small-scale nearwall streaks and large-scale super-streaks was highlighted. The same was also inferred from figure 23, showing the power spectrum. Figure 27 shows visualizations, arranged as sets of three horizontal plots, each pertaining to a different forcing phase $t / T$, at $T^{+}=200$. The left-most plot gives a complete (composite) view of the instantaneous skin friction, while the other two decompose (by means of low-pass/high-pass filtering, see $\S 10)$ the contribution of large and small scales. It is noted, first, that the orientation of the small-scale skin-friction traces align closely with the near-wall streaks. Similarly, when the streaks lose a clear structure and orientation, whilst the shear strain changes direction sufficiently fast, the skin-friction traces follow suit. In contrast, the large-scale skin-friction footprints, separated statistically by around $6 \times 10^{2}$ wall units (i.e. of the same order of the channel half-height), are unaffected in terms of direction.

Figure 28 shows distributions of the phase-averaged variance of the skin-friction fluctuations associated with the large scales, in terms of their relative contributions to the total r.m.s. value. As discussed in $\S 4$, the phase variation of the skin friction is 

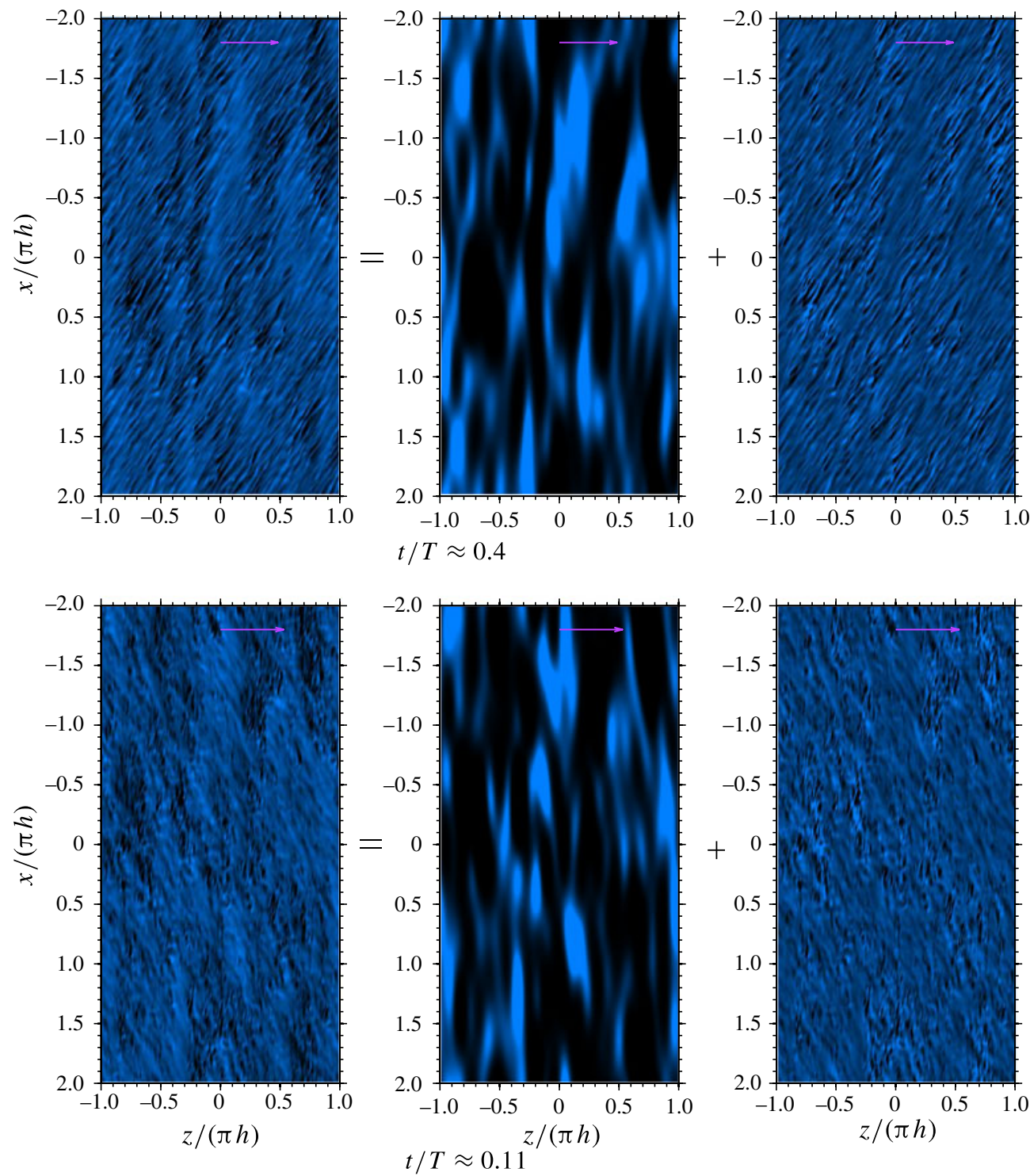

FIGURE 27. (Colour online) Skin-friction-fluctuation decomposition into large- and small-scale contribution.

low, of the order of $1 \%$, certainly low in comparison with its variance, indicated by the PDFs in figure 10. Thus, the variance of total skin friction may be assumed to be virtually insensitive to the phase. In effect, figure 28 gives a quantitative measure of the contributions of the footprints shown in the middle column of visual images in figure 27. As seen, the variations from phase to phase are in the range 24-30\% for $T^{+}=100$ and $12-17 \%$ for $T^{+}=200$. As expected, large-scale structures contribute most to the skin-friction variance when the near-wall streaks are disrupted most strongly, and this occurs when the shear angle undergoes rapid changes between the lingering states at which the streaks are allowed to reform. The high largescale contribution at $T^{+}=100$, in particular, demonstrates that the dynamics of the outer structures plays an increasing important role in the processes dictating the skin 

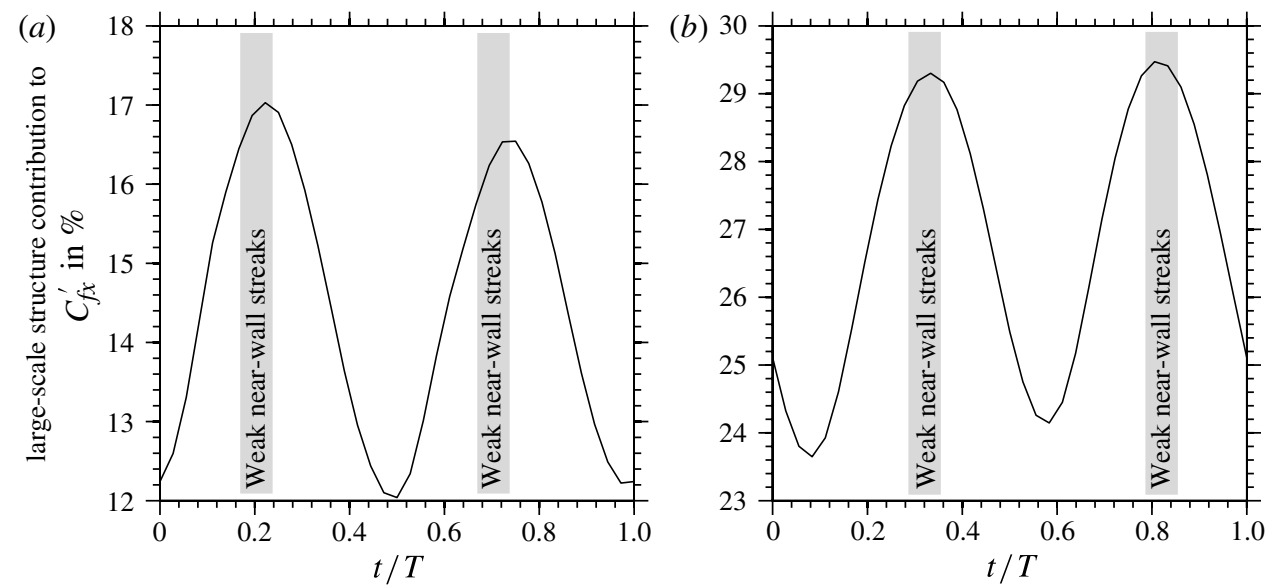

FIGURE 28. Large-scale structures contribution to the total r.m.s. value of the skin-friction fluctuations: (a) $T^{+}=200$; (b) $T^{+}=100$.

friction when forcing minimizes the near-wall streaks. This is likely to become more pronounced with rising Reynolds number.

Another feature that the decomposition in figure 27 brings to light is the significant patchwise alteration of the state of the near-wall streaks, which appears to occur in harmony with large-scale events. More specifically, it is observed that the outer-layer structures, characterized by a velocity excess or velocity defect induce a local phase lag/phase lead in the re-orientation of the small-scale streaks. This process, which may be described as a form of modulation of the near-wall streaks, is accentuated by figure 29 for one particular forcing phase, $t / T=0.11$, and a wall-normal location $y^{+} \approx 10$. In this plot, bright regions are associated with locally elevated streamwise straining due to large-scale structures provoking a positive $u^{\prime}$ component. The phase has been carefully chosen such that the streak orientation established somewhat earlier, during the previous 'strain-lingering' period, is about to 'flip' from the direction identified by the blue arrow to its mirror orientation: see figure $25(a)$. Thus, this is a phase at which the strain angle changes rapidly and at which the streaks are weak and about to enter a period of being reformed at the opposite orientation. In these circumstances, even minor local changes in the strain are likely to induce large changes in the structure of the streaks. As seen in figure 29, the streaks in patches in which the large scales induce elevated streamwise straining undergo an early regeneration and reorientation in the direction of the strain vector, identified by the orange arrow. In these patches, the rate of angular change of the strain vector is decreased, the straining is increased, and the result is a local amplification of newly forming streaks. This complies with the recent observation by Hutchins et al. (2011), where the outer-layer, large-scale and high-velocity structures are found to promote near-wall turbulence. As a consequence, the proportion of the forcing period during which the streaks are weakened by the rapidly changing strain is decreased, and this tends to lower the drag-reduction effectiveness. Naturally, this effect becomes more pronounced with increasing Reynolds number, as observed in DNS for $\operatorname{Re}_{\tau}=1000$ (not included here, except for figure 1), which points to the origin of the lower drag-reduction margins with increasing Reynolds number. 


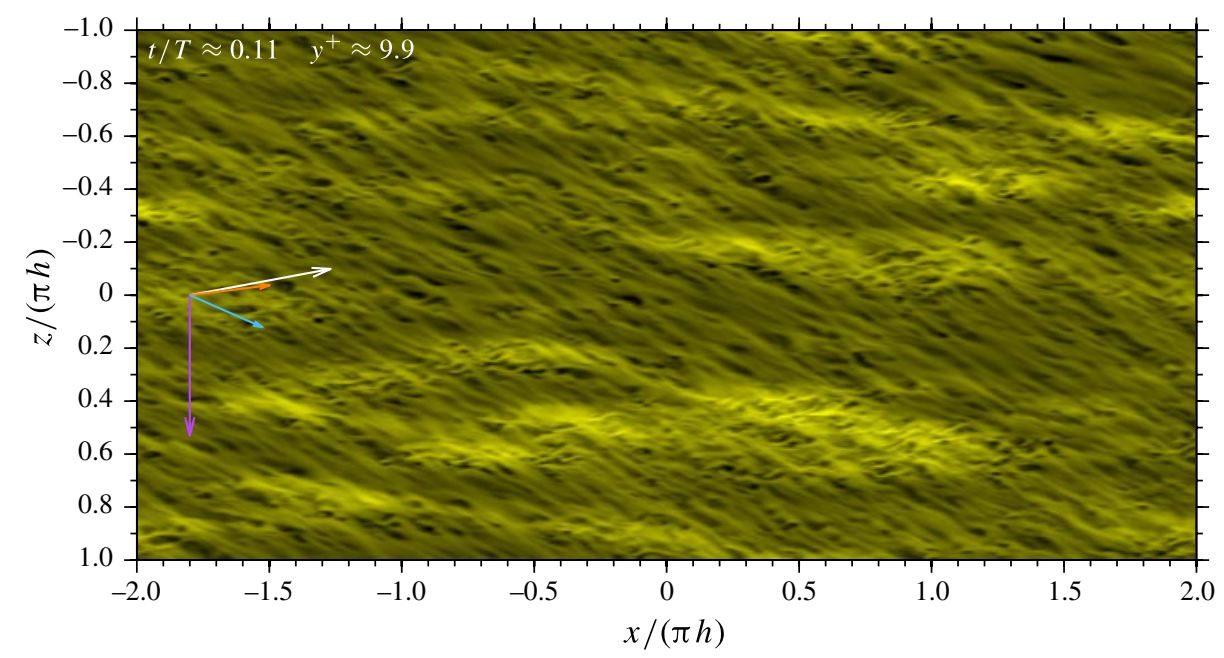

FIGURE 29. (Colour online) Streamwise-velocity fluctuations at $y^{+} \sim 10$, illustrating the effects of outer-layer structures on near-wall streak re-orientation. The presence of largescale high-speed structures is emphasized by brighter contrasts. The vectors show the same quantities as in figure 24, with the addition of the blue vector indicating the orientation angle of the streaky structures outside the bright interaction patches, as shown in figure $25(a)$.

\section{Summary and concluding remarks}

Transverse wall oscillations lead to the formation of a thin, highly sheared Stokes layer, which is observed to cause a significant reduction in streamwise wall friction. The effectiveness of this mechanism depends on the Stokes layer penetration depth, dictated by the oscillation frequency, and hence on the magnitude of the spanwise shear within the viscous sublayer relative to the streamwise shear. For oscillation periods close to the optimum, i.e. that resulting in maximum drag reduction, the Stokes layer is fully confined within the viscous sublayer and does not interact with the turbulence beyond the buffer region. However, a more meaningful aspect of the optimum scenario lies primarily in the ratio between the forcing period and the streak-formation and streak-amplification time scale.

In conditions not far from the optimum, the phase-averaged Stokes motion complies closely with the periodic laminar solution, reflecting a (unidirectional) decoupling of the Stokes motion from the streamwise motion and the turbulence (stochastic) field. However, the Stokes motion has a profound effect on the turbulence field. Thus, statistically, the spanwise motion at near-optimum period causes a drastic reduction in the wall-normal and shear stresses, hence the decline in wall-shear stress and the significant thickening of the viscous sublayer, i.e. the near-wall flow is driven towards a more laminar state. The effect is cumulative, such that the reduced streamwise strain at the wall and the depressed turbulence field are virtually not functions of the forcing phase, despite the periodic Stokes motion. Significant phase fluctuations only arise at non-optimum actuation periods.

All budgets, except that of the spanwise normal stress, reflect a strong attenuation of near-wall turbulence activity. In particular, all contributions in the budgets for the shear stress, the turbulence energy, the streamwise and wall-normal stresses are materially depressed. The budget for the wall-normal stress suggests that the reduction in that stress is driven primarily by an attenuation in wall-normal gradients of pressure fluctuations in the buffer layer, reflecting a suppression of splatting and anti-splatting. 
Associated with this is a reduction in the pressure-velocity interaction process in the buffer layer and, hence, a substantial drop in turbulent diffusion of the stress from the buffer region towards the viscous sublayer. However, these processes only reflect indirect Stokes-motion effects.

The reduction in the wall-normal stress leads to a substantial decline in the generation of the streamwise stress and the shear stress. This is the primary cause for a general depression of the near-wall turbulence, and to some degree, a decorrelation of streamwise and wall-normal fluctuations. Closer examination reveals a disproportionate depletion of the wall-normal fluctuations, relative to the streamwise fluctuations, and that the shear-stress reduction occurs preferentially in the Q2 quadrant, corresponding to ejections, which are particularly affected (depressed) by the forcing.

A direct effect of the Stokes motion is the generation of periodically varying spanwise turbulence fluctuations and hence a substantial elevation of the spanwise normal stress, reflecting the action of a strong generation term that is proportional to the Stokes strain (namely, $v^{\prime \prime} \partial \tilde{w} / \partial y$ ). This causes an elevation of the contribution of the spanwise stress as a proportion of the turbulence energy. Coupled with a reduced streamwise stress, this leads to substantial reduction in anisotropy.

The periodic rise in the spanwise fluctuation, driven by the aforementioned generation term, involving the Stokes strain, combines with the Stokes motion itself to cause major distortions to the streaks, thus disrupting the organized wall-normal motions and the streak structure, eventually inhibiting wall-normal mixing. This process affects primarily the low-speed streaks, as is observed from especially strong reductions in the wall-normal fluctuations and the $u^{\prime \prime}-v^{\prime \prime}$ cross-correlation in near-wall regions into which the distorted low-speed streaks are drawn by the Stokes motion.

The Stokes motion is observed to give rise to a distinct periodicity in the wallparallel structural state of the streaks. There are three states: in two of these, the streaks are organized and inclined in either of two well-defined oblique directions (relatively to the streamwise direction), and in the third they lack any distinct structure. The two inclined states are associated with parts of the forcing periods in which the streamwise strain and Stokes strain combine to form a strain vector that is changing only slowly in time, termed 'lingering'. When the strain 'lingers', the streaks are allowed a sufficient time to form and grow in the direction of the strain vector. For this to take effect, the period of lingering must be comparable to the streak-generation time scale, implied from linear analysis. This time is roughly $50 \mathrm{v} /\left(u_{\tau}^{2}\right)$ (see Blesbois \& Chernyshenko 2011), and concurs with the lingering portion of a forcing period at $T^{+}=200$. When the strain vector changes rapidly, with the spanwise motion changing sign, the streak organization is severely disrupted and the streaks weaken dramatically. If this disruptive state can be maintained throughout the entire actuation cycle, as for $T^{+}=O\left(10^{2}\right)$, the near-wall streaks are virtually suppressed and the lowest level of turbulence skin friction is achieved.

The above process is closely linked to the Stokes strain at $y^{+} \sim 10$, a location at which $v^{\prime \prime} \partial \tilde{w} / \partial y$ reaches its maximum. Despite strong wall-variations of the Stokes strain, and hence strain-vector orientation, the streak orientation is fairly constant across the near-wall layer, being dictated by the shear angle at $y^{+} \sim 10$. That is, there is a pronounced wall-normal coherence in the reoriented streaks, negating the concept of quasi-streamwise vortices playing a key role in the re-orientation process.

The outer-layer large-scale structures are virtually unaffected by the forcing, but contribute significantly to the turbulent skin-friction variance: up to $\sim 30 \%$ in this study, when the near-wall streaks are almost eliminated at the optimum forcing period. 
The large-scale structures are found to directly affect the near-wall streak behaviour. In particular, they augment the strain rate in patches in which they induce positive velocity increments, thus provoking an early reformation of the streaks within phases in which the streaks would otherwise be weakened by high rates of change in the strain-rate angle. This is judged to be the source of the reduced effectiveness of the forcing as the Reynolds number rises and the impact of the outer structures intensifies.

\section{Acknowledgements}

The authors would like to acknowledge the financial support from EPSRC through grant EP/G061556/1, together with Airbus Operations Ltd and EADS UK Ltd. We are also grateful to Imperial College HPC Service facilities for the access to its high-performance computer cx2.

\section{Appendix A. Governing equations for the phase-averaged fluctuations}

Starting from the following triple decomposition

$$
\begin{gathered}
u_{i}=\bar{u}_{i}+\underbrace{\tilde{u}_{i}+u_{i}^{\prime \prime}}_{u_{i}^{\prime}}, \\
p=\bar{p}+\underbrace{\tilde{p}+p^{\prime \prime}}_{p^{\prime}},
\end{gathered}
$$

where the tilde denotes the organized (periodic) fluctuations, the double prime denotes the stochastic fluctuations, and using these in combination with the following governing equations

$$
\begin{gathered}
\frac{\partial u_{k}}{\partial x_{k}}=0, \\
\frac{\partial u_{i}}{\partial t}+u_{k} \frac{\partial u_{i}}{\partial x_{k}}=-\frac{\partial p}{\partial x_{i}}+\frac{1}{R e} \frac{\partial^{2} u_{i}}{\partial x_{k} \partial x_{k}},
\end{gathered}
$$

it is straightforward to show, from (A $1 a$ ) and (A $2 a$ ), that

$$
\frac{\partial \bar{u}_{k}}{\partial x_{k}}=0, \quad \frac{\partial \tilde{u}_{k}}{\partial x_{k}}=0, \quad \frac{\partial u_{k}^{\prime \prime}}{\partial x_{k}}=0 .
$$

Equation (A 1) may be inserted into (A 2b), followed by a combination with (A 3), to yield, after phase-averaging, the result

$$
\begin{aligned}
\frac{\partial \tilde{u}_{i}}{\partial t}+\bar{u}_{k} \frac{\partial \bar{u}_{i}}{\partial x_{k}}+\bar{u}_{k} \frac{\partial \tilde{u}_{i}}{\partial x_{k}}+\tilde{u}_{k} \frac{\partial \bar{u}_{i}}{\partial x_{k}}= & -\frac{\partial \bar{p}}{\partial x_{i}}-\frac{\partial \tilde{p}}{\partial x_{i}}+\frac{1}{R e}\left[\frac{\partial^{2} \bar{u}_{i}}{\partial x_{k} \partial x_{k}}+\frac{\partial^{2} \tilde{u}_{i}}{\partial x_{k} \partial x_{k}}\right] \\
& -\frac{\partial}{\partial x_{k}}\left[\tilde{u}_{i} \tilde{u}_{k}+\left\langle u_{i}^{\prime \prime} u_{k}^{\prime \prime}\right\rangle\right],
\end{aligned}
$$

where

$$
\langle f\rangle \equiv \bar{f}+\tilde{f}
$$

Time-averaging (A 4) gives:

$$
\bar{u}_{k} \frac{\partial \bar{u}_{i}}{\partial x_{k}}=-\frac{\partial \bar{p}}{\partial x_{i}}+\frac{1}{R e} \frac{\partial^{2} \bar{u}_{i}}{\partial x_{k} \partial x_{k}}-\frac{\partial}{\partial x_{k}}\left[\overline{\tilde{u}_{i} \tilde{u}_{k}}+\overline{u_{i}^{\prime \prime} u_{k}^{\prime \prime}}\right] .
$$


Upon subtracting (A 6) from (A 4), the governing equation for the phase fluctuations becomes

$$
\begin{aligned}
\frac{\partial \tilde{u}_{i}}{\partial t}+\bar{u}_{k} \frac{\partial \tilde{u}_{i}}{\partial x_{k}}+\tilde{u}_{k} \frac{\partial \bar{u}_{i}}{\partial x_{k}}= & -\frac{\partial \tilde{p}}{\partial x_{i}}+\frac{1}{\operatorname{Re}} \frac{\partial^{2} \tilde{u}_{i}}{\partial x_{k} \partial x_{k}} \\
& +\frac{\partial}{\partial x_{k}}\left[\overline{\tilde{u}_{i} \tilde{u}_{k}}+\overline{u_{i}^{\prime \prime} u_{k}^{\prime \prime}}-\tilde{u}_{i} \tilde{u}_{k}-\left\langle u_{i}^{\prime \prime} u_{k}^{\prime \prime}\right\rangle\right] .
\end{aligned}
$$

For a double-periodic channel flow, $\bar{v}, \bar{w}, \partial \bar{u}_{i} / \partial x$ and $\partial \bar{u}_{i} / \partial z$ are all strictly zero. In the forced channel, by homogeneity of the forcing in both the streamwise and spanwise directions, phase-averaged fluctuations are also not functions of either $x$ or $z$, i.e. $\partial \tilde{u}_{i} / \partial x=\partial \tilde{u}_{i} / \partial z=0$ or, equivalently, $\tilde{u}_{i}=\tilde{u}_{i}(y, t)$. Therefore, (A 3$)$ yields $\partial \tilde{v} / \partial y=0$ and thus $\tilde{v}=f(t)$. Since $\tilde{v}(y=0, t)=0$ (impermeability at the wall), $f(t)=0$ and

$$
\tilde{v}=0
$$

Therefore, (A 7), reduces to

$$
\begin{aligned}
\frac{\partial \tilde{u}}{\partial t} & =\frac{1}{\operatorname{Re}} \frac{\partial^{2} \tilde{u}}{\partial y^{2}}+\frac{\partial}{\partial y}\left[\overline{u^{\prime \prime} v^{\prime \prime}}-\left\langle u^{\prime \prime} v^{\prime \prime}\right\rangle\right], \\
0 & =-\frac{\partial \tilde{p}}{\partial y}+\frac{\partial}{\partial y}\left[\overline{v^{\prime \prime} v^{\prime \prime}}-\left\langle v^{\prime \prime} v^{\prime \prime}\right\rangle\right], \\
\frac{\partial \tilde{w}}{\partial t} & =\frac{1}{\operatorname{Re}} \frac{\partial^{2} \tilde{w}}{\partial y^{2}}+\frac{\partial}{\partial y}\left[\overline{w^{\prime \prime} v^{\prime \prime}}-\left\langle w^{\prime \prime} v^{\prime \prime}\right\rangle\right] .
\end{aligned}
$$

The two phase-fluctuation components $\tilde{u}$ and $\tilde{w}$ follow a similar governing equation, composed of two distinct contributions: one which represents laminar diffusion and the other associated with the modulation of turbulence fluctuations by the forcing. The laminar part consists of the diffusion of the wall velocity and, therefore, will only apply to the $\tilde{w}$ component (the wall is stationary in the streamwise direction). The turbulence modulation can be important as it is responsible for the additional diffusion of $\tilde{u}$ and $\tilde{w}$. However, this modulation can be relatively small in the present case and it is interesting to estimate its effect on the laminar solution to determine when it is negligible.

To estimate the contribution of the phase-modulated turbulent fluctuations, the following dimensional analysis can be performed. Given the periodic nature of the problem, the relevant time scale is the forcing period $T$, while the relevant velocity scale is the wall velocity $W_{m}$. Given the diffusive nature of the problem, the relevant length scale is the penetration depth $\delta$ over half a cycle, i.e. $\delta \sim \sqrt{v T}$, so that (A $9 c)$ can be written (in dimensional form)

$$
\underbrace{\frac{\partial \tilde{w}}{\partial t}}_{O\left(\frac{W_{m}}{T}\right)}=\underbrace{\nu \frac{\partial^{2} \tilde{w}}{\partial y^{2}}}_{O\left(\frac{W_{m}}{T}\right)}+\underbrace{\frac{\partial}{\partial y}\left[\overline{w^{\prime \prime} v^{\prime \prime}}-\left\langle w^{\prime \prime} v^{\prime \prime}\right\rangle\right.}_{O\left(\frac{\varepsilon U_{b u l k}^{2}}{\sqrt{\nu T}}\right)}]
$$

with

$$
\varepsilon \sim \frac{\overline{w^{\prime \prime} v^{\prime \prime}}-\left\langle w^{\prime \prime} v^{\prime \prime}\right\rangle}{U_{b u l k}^{2}}
$$


where $\varepsilon$ is a parameter quantifying the magnitude of the phase modulation of the turbulence.

For the modulation to be considered negligible, the condition $W_{m} / T \gg \varepsilon U_{b u l k}^{2} / \sqrt{\nu T}$ must be satisfied, which, after a few manipulations, can be written

$$
\varepsilon \ll \frac{W_{m}^{+}}{\sqrt{T^{+}}}\left(\frac{R e_{\tau}}{R e_{\text {bulk }}}\right)^{2} .
$$

For a given channel-flow configuration and a fixed peak wall velocity, $W_{m}$, the constraint on $\varepsilon$ varies as $1 / \sqrt{T^{+}}$and, therefore, the phase fluctuations follow the laminar solution if $T^{+}$is sufficiently small.

\section{Appendix B. Governing equations for the stochastic fluctuations}

Subtracting (A 4) from (A 2b) leads to the governing equation for the stochastic fluctuations:

$$
\begin{aligned}
& \frac{\partial u_{i}^{\prime \prime}}{\partial t}+\bar{u}_{k} \frac{\partial u_{i}^{\prime \prime}}{\partial x_{k}}+\tilde{u}_{k} \frac{\partial u_{i}^{\prime \prime}}{\partial x_{k}}+u_{k}^{\prime \prime} \frac{\partial \bar{u}_{i}}{\partial x_{k}}+u_{k}^{\prime \prime} \frac{\partial \tilde{u}_{i}}{\partial x_{k}} \\
& =-\frac{\partial p^{\prime \prime}}{\partial x_{i}}+\frac{1}{R e} \frac{\partial^{2} u_{i}^{\prime \prime}}{\partial x_{k} \partial x_{k}}+\frac{\partial}{\partial x_{k}}\left[\left\langle u_{i}^{\prime \prime} u_{k}^{\prime \prime}\right\rangle-u_{i}^{\prime \prime} u_{k}^{\prime \prime}\right] .
\end{aligned}
$$

For the double-periodic channel flow discussed in appendix A, (B 1) reduces to

$$
\begin{aligned}
\frac{\partial u^{\prime \prime}}{\partial t} & +\bar{u} \frac{\partial u^{\prime \prime}}{\partial x}+\tilde{u} \frac{\partial u^{\prime \prime}}{\partial x}+\tilde{w} \frac{\partial u^{\prime \prime}}{\partial z}+v^{\prime \prime} \frac{\partial \bar{u}}{\partial y}+v^{\prime \prime} \frac{\partial \tilde{u}}{\partial y} \\
= & -\frac{\partial p^{\prime \prime}}{\partial x}+\frac{1}{R e}\left[\frac{\partial^{2} u^{\prime \prime}}{\partial x^{2}}+\frac{\partial^{2} u^{\prime \prime}}{\partial y^{2}}+\frac{\partial^{2} u^{\prime \prime}}{\partial z^{2}}\right] \\
& -\frac{\partial u^{\prime \prime} u^{\prime \prime}}{\partial x}-\frac{\partial u^{\prime \prime} w^{\prime \prime}}{\partial z}+\frac{\partial}{\partial y}\left[\left\langle u^{\prime \prime} v^{\prime \prime}\right\rangle-u^{\prime \prime} v^{\prime \prime}\right], \\
\frac{\partial v^{\prime \prime}}{\partial t} & +\bar{u} \frac{\partial v^{\prime \prime}}{\partial x}+\tilde{u} \frac{\partial v^{\prime \prime}}{\partial x}+\tilde{w} \frac{\partial v^{\prime \prime}}{\partial z} \\
= & -\frac{\partial p^{\prime \prime}}{\partial y}+\frac{1}{R e}\left[\frac{\partial^{2} v^{\prime \prime}}{\partial x^{2}}+\frac{\partial^{2} v^{\prime \prime}}{\partial y^{2}}+\frac{\partial^{2} v^{\prime \prime}}{\partial z^{2}}\right] \\
& -\frac{\partial v^{\prime \prime} u^{\prime \prime}}{\partial x}-\frac{\partial v^{\prime \prime} w^{\prime \prime}}{\partial z}+\frac{\partial}{\partial y}\left[\left\langle v^{\prime \prime} v^{\prime \prime}\right\rangle-v^{\prime \prime} v^{\prime \prime}\right], \\
\frac{\partial w^{\prime \prime}}{\partial t} & +\bar{u} \frac{\partial w^{\prime \prime}}{\partial x}+\tilde{u} \frac{\partial w^{\prime \prime}}{\partial x}+\tilde{w} \frac{\partial w^{\prime \prime}}{\partial z}+v^{\prime \prime} \frac{\partial \tilde{w}}{\partial y} \\
= & -\frac{\partial p^{\prime \prime}}{\partial z}+\frac{1}{R e}\left[\frac{\partial^{2} w^{\prime \prime}}{\partial x^{2}}+\frac{\partial^{2} w^{\prime \prime}}{\partial y^{2}}+\frac{\partial^{2} w^{\prime \prime}}{\partial z^{2}}\right]-\frac{\partial w^{\prime \prime} u^{\prime \prime}}{\partial x} \\
& -\frac{\partial w^{\prime \prime} w^{\prime \prime}}{\partial z}+\frac{\partial}{\partial y}\left[\left\langle w^{\prime \prime} v^{\prime \prime}\right\rangle-w^{\prime \prime} v^{\prime \prime}\right] .
\end{aligned}
$$

If $T^{+}$is sufficiently small so that the condition in (A 12) is satisfied, any contribution

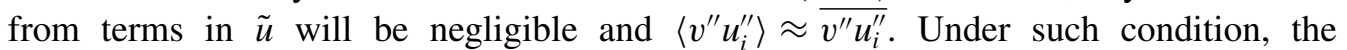
stochastic fluctuations follow:

$$
\frac{\partial u^{\prime \prime}}{\partial t}+\underbrace{\bar{u} \frac{\partial u^{\prime \prime}}{\partial x}}_{(\text {mconv })}+\underbrace{u_{i}^{\prime \prime} \frac{\partial u^{\prime \prime}}{\partial x_{i}}}_{(\text {tconv })}+\underbrace{\tilde{w} \frac{\partial u^{\prime \prime}}{\partial z}}_{(\text {sconv })} \approx \underbrace{-\frac{\partial p^{\prime \prime}}{\partial x}}_{(\text {redi })}+\underbrace{\frac{1}{R e} \frac{\partial^{2} u^{\prime \prime}}{\partial x_{i} \partial x_{i}}+\frac{\mathrm{d} \overline{u^{\prime \prime} v^{\prime \prime}}}{\mathrm{d} y}}_{(\text {diff })} \underbrace{-v^{\prime \prime} \frac{\mathrm{d} \bar{u}}{\mathrm{~d} y}}_{(i)},(\mathrm{B} 3 a)
$$


Near-wall streak modifications by spanwise oscillatory wall motions

$$
\begin{gathered}
\frac{\partial v^{\prime \prime}}{\partial t}+\underbrace{\bar{u} \frac{\partial v^{\prime \prime}}{\partial x}}_{(\text {mconv })}+\underbrace{u_{i}^{\prime \prime} \frac{\partial v^{\prime \prime}}{\partial x_{i}}}_{(\text {tconv })}+\underbrace{\tilde{w} \frac{\partial v^{\prime \prime}}{\partial z}}_{(\text {sconv })} \approx \underbrace{-\frac{\partial p^{\prime \prime}}{\partial y}}_{(\text {redi })}+\underbrace{\frac{1}{R e} \frac{\partial^{2} v^{\prime \prime}}{\partial x_{i} \partial x_{i}}+\frac{\mathrm{d} \overline{v^{\prime \prime} v^{\prime \prime}}}{\mathrm{d} y}}_{(\text {diff })} \\
\frac{\partial w^{\prime \prime}}{\partial t}+\underbrace{\bar{u} \frac{\partial w^{\prime \prime}}{\partial x}}_{(\text {mconv })}+\underbrace{u_{i}^{\prime \prime} \frac{\partial w^{\prime \prime}}{\partial x_{i}}}_{(\text {tconv })}+\underbrace{\tilde{w} \frac{\partial w^{\prime \prime}}{\partial z}}_{(\text {sconv })} \approx \underbrace{-\frac{\partial p^{\prime \prime}}{\partial z}}_{(\text {redi })}+\underbrace{\frac{1}{\operatorname{Re} \frac{\partial^{2} w^{\prime \prime}}{\partial x_{i} \partial x_{i}}}+\frac{\mathrm{d} \overline{w^{\prime \prime} v^{\prime \prime}}}{\mathrm{d} y}}_{(\text {diff })} \underbrace{-v^{\prime \prime} \frac{\partial \tilde{w}}{\partial y}}_{(\text {ii })} .
\end{gathered}
$$

As expected, the stochastic fluctuations are convected by the total velocity field and, in particular, the Stokes layer (denoted by (sconv)). They are also diffused through both viscous and turbulent diffusion (englobed by (diff)). The pressure-gradient terms, (redi), redistribute the fluctuations such that mass conservation is enforced. Of particular interest are the terms labelled (i) and (ii). The former is associated with the production of streamwise velocity fluctuations through the interaction of wallnormal velocity fluctuations with the mean streamwise velocity wall-normal gradient. The latter term is mathematically similar to the former but involves the production of spanwise velocity fluctuations through the interaction of wall-normal velocity fluctuations with the unsteady straining due to the Stokes layer. The role played by ( $i$ ) in the generation cycle of near-wall streak is described for example in Chernyshenko \& Baig (2005). The mathematical similarity of (ii) is fundamentally important to the generation of the new, oblique structures described in this paper.

\section{Appendix C. Reynolds-stress budget derivations for the stochastic fluctuations}

Upon multiplying (B 1 ) by $u_{j}^{\prime \prime}$, then adding the result to itself with the $i$ and $j$ indices swapped, it is possible to write (after a few manipulations and using (A 3))

$$
\begin{aligned}
& \frac{\partial u_{i}^{\prime \prime} u_{j}^{\prime \prime}}{\partial t}+\left\langle u_{k}\right\rangle \frac{\partial u_{i}^{\prime \prime} u_{j}^{\prime \prime}}{\partial x_{k}}+u_{j}^{\prime \prime} u_{k}^{\prime \prime} \frac{\partial\left\langle u_{i}\right\rangle}{\partial x_{k}}+u_{i}^{\prime \prime} u_{k}^{\prime \prime} \frac{\partial\left\langle u_{j}\right\rangle}{\partial x_{k}}+\frac{\partial u_{i}^{\prime \prime} u_{j}^{\prime \prime} u_{k}^{\prime \prime}}{\partial x_{k}} \\
& =-u_{i}^{\prime \prime} \frac{\partial p^{\prime \prime}}{\partial x_{j}}-u_{j}^{\prime \prime} \frac{\partial p^{\prime \prime}}{\partial x_{i}}+u_{i}^{\prime \prime} \frac{\partial\left\langle u_{j}^{\prime \prime} u_{k}^{\prime \prime}\right\rangle}{\partial x_{k}}+u_{j}^{\prime \prime} \frac{\partial\left\langle u_{i}^{\prime \prime} u_{k}^{\prime \prime}\right\rangle}{\partial x_{k}}+\frac{1}{\operatorname{Re}} \frac{\partial^{2} u_{i}^{\prime \prime} u_{j}^{\prime \prime}}{\partial x_{k} \partial x_{k}}-\frac{2}{\operatorname{Re}} \frac{\partial u_{i}^{\prime \prime}}{\partial x_{k}} \frac{\partial u_{j}^{\prime \prime}}{\partial x_{k}}
\end{aligned}
$$

which can then be phase-averaged to obtain

$$
\begin{aligned}
& \frac{\partial\left\langle u_{i}^{\prime \prime} u_{j}^{\prime \prime}\right\rangle}{\partial t}+\left\langle u_{k}\right\rangle \frac{\partial\left\langle u_{i}^{\prime \prime} u_{j}^{\prime \prime}\right\rangle}{\partial x_{k}}+\frac{\partial\left\langle u_{i}^{\prime \prime} u_{j}^{\prime \prime} u_{k}^{\prime \prime}\right\rangle}{\partial x_{k}} \\
& =-\left\langle u_{i}^{\prime \prime} \frac{\partial p^{\prime \prime}}{\partial x_{j}}\right\rangle-\left\langle u_{j}^{\prime \prime} \frac{\partial p^{\prime \prime}}{\partial x_{i}}\right\rangle-\left\langle u_{j}^{\prime \prime} u_{k}^{\prime \prime}\right\rangle \frac{\partial\left\langle u_{i}\right\rangle}{\partial x_{k}}-\left\langle u_{i}^{\prime \prime} u_{k}^{\prime \prime}\right\rangle \frac{\partial\left\langle u_{j}\right\rangle}{\partial x_{k}} \\
& \quad-\frac{2}{R e}\left\langle\frac{\partial u_{i}^{\prime \prime}}{\partial x_{k}} \frac{\partial u_{j}^{\prime \prime}}{\partial x_{k}}\right\rangle+\frac{1}{R e} \frac{\partial^{2}\left\langle u_{i}^{\prime \prime} u_{j}^{\prime \prime}\right\rangle}{\partial x_{k} \partial x_{k}},
\end{aligned}
$$

where we have made use of: (i) the commutativity between the derivative and averaging operators, i.e. $\langle\partial f / \partial[\cdot]\rangle=\partial\langle f\rangle / \partial[\cdot]$; (ii) the constant-preserving property of the averaging operator, i.e. $\langle c f\rangle=c\langle f\rangle,\langle\langle f\rangle\rangle=\langle f\rangle$; (iii) the definition of the stochastic fluctuations, i.e. $\left\langle f^{\prime \prime}\right\rangle=0$. 
The phase-averaged equation is then time-averaged to give

$$
\begin{aligned}
\bar{u}_{k} \frac{\partial \overline{u_{i}^{\prime \prime} u_{j}^{\prime \prime}}}{\partial x_{k}}+\overline{\tilde{u}_{k} \frac{\partial\left\langle u_{i}^{\prime \prime} u_{j}^{\prime \prime}\right\rangle}{\partial x_{k}}}+\frac{\partial \overline{u_{i}^{\prime \prime} u_{j}^{\prime \prime} u_{k}^{\prime \prime}}}{\partial x_{k}} \\
=-\overline{u_{i}^{\prime \prime} \frac{\partial p^{\prime \prime}}{\partial x_{j}}}-\overline{u_{j}^{\prime \prime}} \frac{\partial p^{\prime \prime}}{\partial x_{i}}-\overline{u_{j}^{\prime \prime} u_{k}^{\prime \prime}} \frac{\partial \bar{u}_{i}}{\partial x_{k}}-\overline{u_{i}^{\prime \prime} u_{k}^{\prime \prime}} \frac{\partial \bar{u}_{j}}{\partial x_{k}} \\
\quad-\overline{\left\langle u_{j}^{\prime \prime} u_{k}^{\prime \prime}\right\rangle \frac{\partial \tilde{u}_{i}}{\partial x_{k}}}-\overline{\left\langle u_{i}^{\prime \prime} u_{k}^{\prime \prime}\right\rangle \frac{\partial \tilde{u}_{j}}{\partial x_{k}}}-\frac{2}{\operatorname{Re}} \overline{\frac{\partial u_{i}^{\prime \prime}}{\partial x_{k}} \frac{\partial u_{j}^{\prime \prime}}{\partial x_{k}}}+\frac{1}{\operatorname{Re} \frac{\partial^{2}}{\partial u_{i}^{\prime \prime} u_{j}^{\prime \prime}}},
\end{aligned}
$$

where we have made use of: (i) the commutativity between the derivative and averaging operators, i.e. $\overline{\partial f / \partial[\cdot]}=\partial \bar{f} / \partial[\cdot]$; (ii) the constant-preserving property of the averaging operator, i.e. $\overline{c f}=c \bar{f}, \overline{\bar{f}}=\bar{f}$; (iii) $\overline{\left\langle f^{\prime \prime} g^{\prime \prime}\right\rangle}=\overline{f^{\prime \prime} g^{\prime \prime}}$.

Applying (C 3) to the double-periodic channel gives (7.1).

\section{REFERENCES}

Adrian, R. J., Meinhart, C. D. \& Tomkins, C. D. 2000 Vortex organization in the outer region of the turbulent boundary layer. J. Fluid Mech. 422, 1-54.

Adrian, R. J., Meinhart, C. D. \& Tomkins, C. D. 2000 Vortex organization in the outer region of the turbulent boundary layer. J. Fluid Mech. 422, 1-54.

Akhavan, R., Jung, W. J. \& MangiavacChi, N. 1993 Turbulence control in wall-bounded flows by spanwise oscillations. Appl. Sci. Res. 51, 299-303.

BAron, A. \& QuAdrio, M. 1995 Turbulent drag reduction by spanwise wall oscillations. Appl. Sci. Res. 55, 311-326.

Blesbois, O. \& Chernyshenko, S. I. 2011 Generalised optimal perturbation approach applied to drag reduction by wall oscillations in turbulent flows. In 7 th International Symposium on Turbulence and Shear Flow Phenomena, Ottawa, pp. 1-6. Paper is available from TSFP-7 website (http://tsfp7.org/papers.php).

BRAdshaw, P. \& Pontikos, P. 1985 Measurements in the turbulent boundary layer on an 'infinite' swept wing. J. Fluid Mech. 159, 105-130.

CHERnyshenko, S. I. \& BAIG, M. F. 2005 The mechanism of streak formation in near-wall turbulence. J. Fluid Mech. 544, 99-131.

CHOI, K.-S. 2002 Near-wall structure of turbulent boundary layer with spanwise-wall oscillation. Phys. Fluids 14 (7), 2530-2542.

Choi, K.-S. \& Clayton, B. R. 2001 The mechanism of turbulent drag reduction with wall oscillation. Intl J. Heat Fluid Flow 22 (1), 1-9.

Choi, K.-S., DeBisschop, J.-R. \& Clayton, B. R. 1998 Turbulent boundary-layer control by means of spanwise-wall oscillation. AIAA J. 36 (7).

CHOI, K.-S. \& GRAHAM, M. 1998 Drag reduction of turbulent pipe flows by circular-wall oscillation. Phys. Fluids 10 (1).

Choi, H., Moin, P. \& KIM, J. 1994 Active turbulence control for drag reduction in wall-bounded flows. J. Fluid Mech. 262, 75-110.

Choi, J.-I., XU, C.-X. \& Sung, H.-J. 2002 Drag reduction by spanwise wall oscillation in wall-bounded turbulent flows. AIAA J. 40 (5).

DHANAK, M. R. \& SI, C. 1999 On reduction of turbulent wall friction through spanwise wall oscillations. J. Fluid Mech. 383, 175-195.

Di Cicca, G. M., Iuso, G., Spazzini, P. G. \& Onorato, M. 2002 Particle image velocimetry investigation of a turbulent boundary layer manipulated by spanwise wall oscillations. J. Fluid Mech. 467, 41-56.

Driver, D. M. \& HebBaR, S. K. 1987 Experimental study of a three-dimensional shear-driven, turbulent boundary layer. AIAA J. 25 (1).

Du, Y., Symeonidis, V. \& Karniadakis, G. E. 2002 Drag reduction in wall-bounded turbulence via a transverse travelling wave. J. Fluid Mech. 457, 1-34. 
EAton, J. K. 1995 Effects of mean flow three dimensionality on turbulent boundary-layer structure. AIAA J. 33 (11).

Fishrool, G. M. \& Leschziner, M. A. 2009 Stability bounds for explicit fractional-step schemes for the Navier-Stokes equations at high Reynolds number. Comput. Fluids 38, 1289-1298.

Fröhlich, J., Mellen, C. P., Rodi, W., Temmerman, L. \& Leschziner, M. A. 2005 Highly resolved large-eddy simulations of seperated flow in a channel with streamwise periodic constrictions. J. Fluid Mech. 526, 19-66.

Frohnapfel, B., Hasegawa, Y. \& Kasagi, N. 2010 Friction drag reduction through damping of the near-wall spanwise velocity fluctuation. Intl J. Heat Fluid Flow 31 (3), 434-441.

Frohnapfel, B., Lammers, P., Jovanović, J. \& Durst, F. 2007 Interpretation of the mechanism associated with turbulent drag reduction in terms of anisotropy invariants. J. Fluid Mech. 577, 457-466.

Fukagata, K., Iwamoto, K. \& Kasagi, N. 2002 Contribution of Reynolds stress distribution to the skin friction in wall-bounded flows. Phys. Fluids 14 (11).

Fukagata, K., Kobayashi, M. \& Kasagi, N. 2010 On the friction drag reduction effect by a control of large-scale turbulent structures. J. Fluid Sci. Technol. 5 (3).

Hamilton, J. M., Kim, J \& WalefFe, F. 1995 Regeneration mechanisms of near-wall turbulence structures. J. Fluid Mech. 287, 317-348.

Howard, R. J. A \& SANDham, N. D. 2000 Simulation and modelling of a skewed turbulent channel flow. Flow Turbul. Combust. 65, 83-109.

Hutchins, N., Monty, J. P., Ganapathisubramani, B., NG, H. C. H \& Marusic, I. 2011 Three-dimensional conditional structure of a high-Reynolds-number turbulent boundary layer. J. Fluid Mech. 673, 255-285.

Jimenez, J. \& Pinelli, A. 1999 The autonomous cycle of near-wall turbulence. J. Fluid Mech. 389, 335-359.

Jung, W. J., Mangiavacchi, N. \& Akhavan, R. 1992 Suppression of turbulence in wall-bounded flows by high-frequency spanwise oscillations. Phys. Fluids A 4 (8), 1605-1607.

KAng, S. \& CHOI, H. 2000 Active wall motions for skin-friction drag reduction. Phys. Fluids 12 (12).

KARNIADAKIS, G. E. \& CHOI, K.-S. 2003 Mechanisms on transverse motions in turbulent wall flows. Annu. Rev. Fluid Mech. 35, 45-62.

KIM, J. 2011 Physics and control of wall turbulence for drag reduction. Phil. Trans. R. Soc. A 369 (1940), 1396-1411.

Kim, H. T., KLine, S. J. \& ReYnolds, W. C. 1971 The production of turbulence near a smooth wall in a turbulent boundary layer. J. Fluid Mech. 50, 133-160.

Kline, S. J., Reynolds, W. C., Schraub, F. A. \& Runstadler, P. W. 1967 The structure of turbulent boundary layers. J. Fluid Mech. 30, 741-773.

Kravchenko, A., Choi, H. \& Moin, P. 1993 On the relation of near wall streamwise vortices to wall skin friction in turbulent boundary layersriction drag reduction achievable by near-wall turbulence manipulation at high Reyolds numbers. Phys. Fluids 5 (12).

Kreplin, H.-P. \& ECKelmanN, H. 1979 Propagation of perturbations in the viscous sublayer and adjacent wall region. J. Fluid Mech. 95, 305-322.

LAadhari, F., SkAndaji, L. \& Morel, R. 1994 Turbulence reduction in a boundary layer by a local spanwise oscillating surface. Phys. Fluids 6 (10).

Lee, C., Kim, J., BАвсоск, D. \& Goodman, R. 1997 Application of neural networks to turbulence control for drag reduction. Phys. Fluids 9 (6).

Lockerby, D. A., Carpenter, P. W. \& Davies, C. 2005 Control of sublayer streaks using microjet actuators. AIAA J. 43 (9).

Marusic, I., Mathis, R. \& Hutchins, N. 2010 Predictive model for wall-bounded turbulent flow. Science 329, 193-196.

Moser, R. D., Kim, J. \& MAnsour, N. N. 1999 Direct numerical simulation of turbulent channel flow up to $R e_{\tau}=590$. Phys. Fluids 11 (4), 943-945.

PANG, J. \& CHOI, K.-S. 2004 Turbulent drag reduction by Lorentz force oscillation. Phys. Fluids 16 (5). 
PAnton, R. L. 1997 Self-sustaining mechanisms of wall turbulence. Comput. Mech. 422 pages. http://cdsweb.cern.ch/record/489684.

QUADRIO, M. \& RICCO, P. 2003 Initial response of a turbulent channel flow to spanwise oscillation of the walls. J. Turbul. 4 (7).

QUADRIO, M. \& RICCO, P. 2004 Critical assessment of turbulent drag reduction through spanwise wall oscillations. J. Fluid Mech. 521, 251-271.

QUADRIO, M. \& RicCO, P. 2011 The laminar generalized stokes layer and turbulent drag reduction. J. Fluid Mech. 667, 135-157.

QUADRIo, M., Ricco, P. \& ViotTi, C. 2009 Streamwise-travelling waves of spanwise wall velocity for turbulent drag reduction. J. Fluid Mech. 627, 161-178.

QuAdrio, M. \& Sibilla, S. 2000 Numerical simulation of turbulent flow in a pipe oscillating around its axis. J. Fluid Mech. 424, 217-241.

Rathnasingham, R. \& BREUER, K. S. 1997 System identification and control of a turbulent boundary layer. Phys. Fluids 9 (7).

Rhie, C. M. \& CHOw, W. L. 1983 Numerical study of the turbulent flow past an aerofoil with trailing edge separation. AIAA J. 21 (11).

RICCO, P. 2004 Modification of near-wall turbulence due to spanwise wall oscillations. J. Turbul. 5 (24).

RicCO, P. \& QUADRIO, M. 2008 Wall-oscillation conditions for drag reduction in turbulent channel flow. Intl J. Heat Fluid Flow 29 (4), 891-902.

SCHOPPA, W. \& HuSSAIN, F. 2002 Coherent structure generation in near-wall turbulence. J. Fluid Mech. 453, 57-108.

Schraub, F. A. \& KLine, S. J. 1965 A study of the structure of the turbulent boundary layer with and without longitudinal pressure gradients. Tech. Rep. MD-12. Department of Mechanical Engineering, Stanford University.

Sendstad, O. \& Moin, P. 1992 The near wall mechanics of three-dimensional turbulent boundary layers. Tech. Rep. TF-57. Department of Mechanical Engineering, Stanford University.

Spalart, P. 1986 Numerical study of sink-flow boundary layers. J. Fluid Mech. 172, 307-328.

Temmerman, L., Leschziner, M. A., Mellen, C. P. \& Fröhlich, J. 2003 Investigation of wall-function approximations and subgrid-scale models in large eddy simulation of separated flow in a channel with streamwise periodic constrictions. Intl J. Heat Fluid Flow 24 (2), $157-180$.

Touber, E. \& LeschZIner, M. A. 2011 Near-wall streak modification by spanwise oscillatory wall motions. In 7th International Symposium on Turbulence and Shear Flow Phenomena, Ottawa, pp. 1-6. Paper available for download from TSFP-7 website (http://tsfp7.org/papers. php).

WALEFFE, F. \& KIM, J. 1997 How streamwise rolls and streaks self-sustain in a shear flow. Adv. Fluid Mech. Ser. 15, 309-332.

XU, C.-X. \& HUANG, W.-X. 2005 Transient response of Reynolds stress transport to spanwise wall oscillation in a turbulent channel flow. Phys. Fluids 17 (1).

Yakeno, A., Hasegawa, Y. \& Kasagi, N. 2010 Interpretation of the optimal frequency for skin friction drag reduction with spanwise wall-oscillation control. In 8th International ERCOFTAC Symposium on Engineering Turbulence Modelling and Measurements, Marseille, pp. 356-361. ERCOFTAC.

Yoshino, T., SuzuKi, Y. \& KASAGI, N. 2003 Evaluation of GA-based feedback control system for drag reduction in wall turbulence. In Proceedings of 3rd International Symposium on Turbulence and Shear Flow Phenomena, Sendai, Japan, pp. 179-184.

Zhou, J., Adrian, R. J., Balachandar, S. \& Kendall, T. M. 1999 Mechanisms for generating coherent packets of hairpin vortices in channel flow. J. Fluid Mech. 387, 353-396. 